\title{
GENERALIZED BLOW-UP OF CORNERS AND FIBER PRODUCTS
}

\author{
CHRIS KOTTKE AND RICHARD B. MELROSE
}

\begin{abstract}
Real blow-up, including inhomogeneous versions, of boundary faces of a manifold (with corners) is an important tool for resolving singularities, degeneracies and competing notions of homogeneity. These constructions are shown to be particular cases of generalized boundary blow-up in which a new manifold and blow-down map are constructed from, and conversely determine, combinatorial data at the boundary faces in the form of a refinement of the basic monoidal complex of the manifold. This data specifies which notion of homogeneity is realized at each of the boundary hypersurfaces in the blown-up space.

As an application of this theory, the existence of fiber products is examined for the natural smooth maps in this context, the b-maps. Transversality of the b-differentials is shown to ensure that the set-theoretic fiber product of two maps is a binomial variety. Properties of these (extrinsically defined) spaces, which generalize manifolds but have mild singularities at the boundary, are investigated, and a condition on the basic monoidal complex is found under which the variety has a smooth structure. Applied to b-maps this additional condition with transversality leads to a universal fiber product in the context of manifolds with corners. Under the transversality condition alone the fiber product is resolvable to a smooth manifold by generalized blow-up and then has a weaker form of the universal mapping property requiring blow-up of the domain.
\end{abstract}

\section{INTRODUCTION}

Real blow-up of a submanifold introduces a new manifold (always here implicitly meaning 'with corners') in which the submanifold in question is replaced by one or more boundary hypersurfaces in a prescribed manner. Here we introduce the notion of the generalized boundary blow-up of a manifold with corners, $Y$, which is a new manifold $X$ along with a smooth and proper 'blow-down' map $\beta: X \longrightarrow Y$. The latter is by definition a b-map, restricting to a diffeomorphism on the interiors, with bijective b-differential. It includes standard radial blow-up of boundary faces, iterated boundary blow-up, and inhomogeneous blow-up as special cases.

It is shown here that such blow-down maps are, up to diffeomorphism, characterized in an essentially algebraic manner by a 'monoidal complex', a consistent choice of certain combinatorial data at each face of $Y$. One application of the constructive part of this result is to fiber products of maps. Working in the category of smooth manifolds and b-maps, it is shown that under the natural condition of

Received by the editors April 10, 2012 and, in revised form, May 15, 2013.

2010 Mathematics Subject Classification. Primary 57R99; Secondary 14B05.

Key words and phrases. Manifold with corners, blow-up, generalized blow-up, fiber product, monoidal complex.

The second author was supported in part by NSF grant DMS-1005944. 
b-transversality the fiber product of two maps can be decomposed and smoothed by generalized blow-up, and this resolved fiber product has a weakened form of the universal factoring property for fiber products in which generalized blow-up of the domain may be required.

Although this paper is concerned with geometric and algebraic questions, one of the contexts in which blow-up appears is analytic. Most analytic problems on a manifold with corners do not have solutions 'within the smooth category'. For instance, harmonic forms with absolute or relative boundary conditions are not typically smooth up to corners. Rather they lie in conormal spaces which themselves are only 'resolved' to polyhomogeneity by blow-up. Here polyhomogeneity is to be thought of as the natural extension of smoothness, in which $C^{\infty}(Y)$ is extended to include functions which have non-integral (including possibly negative) powers in their 'Taylor series' at the boundary. The selection of the correct resolution, in the sense of blow-up, of spaces and their (fiber) products, on which the kernels of operators are defined, is one of the prime motivations for the discussion here.

A manifold (meaning from now on a manifold with corners) $Y$ is a topological manifold with boundary which is locally diffeomorphic to the model space $[0, \infty)^{k} \times$ $\mathbb{R}^{n-k}$ with its sheaf of smooth functions. The set of (connected) boundary faces of $Y$ is denoted $\mathcal{M}(Y)$, with $\mathcal{M}_{l}(Y)$ denoting those faces of codimension $l \in\{0, \ldots, k\}$. We require that boundary hypersurfaces of a manifold be embedded, which means that the functions vanishing on each such hypersurface form a principal ideal $\mathcal{I}_{H} \subset$ $C^{\infty}(Y), H \in \mathcal{M}_{1}(Y)$. A smooth map $f: X \longrightarrow Y$ is an (interior) boundary map or 'b-map' as defined in Mel, Mel92 provided it pulls back each of these principal ideals to a product

$$
f^{*} \mathcal{I}_{H}=\prod_{G \in \mathcal{M}_{1}(Y)} \mathcal{I}_{G}^{\alpha(H, G)}, \quad \alpha(\cdot, \cdot) \in \mathbb{N}
$$

of similar ideals in $C^{\infty}(X)$.

For each face $F \in \mathcal{M}_{l}(X)$ the freely generated monoid

$$
\sigma_{F}=\bigoplus_{H \in \mathcal{M}_{1}(X), F \subseteq H} \mathbb{N} e_{H}
$$

along with the inclusions $i_{G F}: \sigma_{G} \longleftrightarrow \sigma_{F}$ for $F \subset G$ constitute what we call the 'basic monoidal complex' of $X$ :

$$
\mathcal{P}_{X}=\left\{\left(\sigma_{F}, i_{G F}\right) ; F \subseteq G \in \mathcal{M}(X)\right\},
$$

and a b-map $f: X \longrightarrow Y$ defines a morphism of the complexes

$$
\begin{aligned}
& f_{\natural}: \mathcal{P}_{X} \longrightarrow \mathcal{P}_{Y}, \\
& \sigma_{F} \stackrel{f_{\natural}}{\longrightarrow} \sigma_{f_{\#}(F)},
\end{aligned}
$$

which is fundamental to our discussion. Here $f_{\#}: \mathcal{M}(X) \longrightarrow \mathcal{M}(Y)$ maps each boundary face of $X$ to the boundary face of the largest codimension in $Y$ which contains its image, and the coefficients of $f_{\natural}: \sigma_{F} \longrightarrow \sigma_{f_{\#}(F)}$ are the relevant exponents $\alpha(\cdot, \cdot) \in \mathbb{N}$ occuring in (I.1).

In general, the monoidal complexes and their morphisms capture only limited combinatorial information regarding the boundary faces of $X$ and $Y$. However in certain special cases, such as when $X$ is the blow-up of a boundary face $F$ in $Y$, this information is enough to completely specify, up to diffeomorphism, the domain $X=[Y ; F]$ and the map $\beta: X \longrightarrow Y$ in terms of the range space $Y$. In such a case 
the morphism (I.2) forms what is called below a 'smooth refinement' of $\mathcal{P}_{Y}$, and on the analytical side $\beta$ satisfies additional properties, namely

$$
\begin{gathered}
\beta: X \backslash \partial X \longrightarrow Y \backslash \partial Y \text { is a diffeomorphism and } \\
{ }^{\mathrm{b}} \beta_{*}:{ }^{\mathrm{b}} T_{p} X \longrightarrow{ }^{\mathrm{b}} T_{\beta(p)} Y \text { is an isomorphism for all } p \in X,
\end{gathered}
$$

where ${ }^{\mathrm{b}} \beta_{*}$ is the differential acting on the b-tangent bundles.

Our first main result consists of two parts: a generalization of this 'boundary blow-up' with respect to arbitrary smooth refinements of $\mathcal{P}_{Y}$, and an analytical characterization of such a map.

Theorem A. To any smooth refinement $\mathcal{R} \longrightarrow \mathcal{P}_{Y}$ there corresponds a manifold denoted $X=[Y ; \mathcal{R}]$, unique up to diffeomorphism and with $\mathcal{P}_{X}=\mathcal{R}$, and a unique b-map $f: X=[Y ; \mathcal{R}] \longrightarrow Y$ satisfying (I.3) and (I.4). Conversely, any smooth proper b-map satisfying (I.3) and (I.4) determines a smooth refinement $\mathcal{P}_{X} \longrightarrow \mathcal{P}_{Y}$.

The manifold $[Y ; \mathcal{R}]$ is referred to as the 'generalized blow-up' of $Y$ by the refinement $\mathcal{R}$, and the lifting problem for b-maps under generalized blow-ups of the domain and/or range is resolved by examining the corresponding lifting of the monoidal complex morphisms.

Our second main result is an application of this theory to fiber products. Recall that a (smooth) fiber product of two maps $f_{i}: X_{i} \longrightarrow Y, i=1,2$, does not generally exist, even in the category of smooth manifolds without boundary. There is however a sufficient condition for existence, namely that $f_{1}$ and $f_{2}$ be transversal, meaning that if $f_{1}\left(p_{1}\right)=f_{2}\left(p_{2}\right)=q \in Y$, then

$$
\left(f_{1}\right)_{*}\left(T_{p_{1}} X_{1}\right)+\left(f_{2}\right)_{*}\left(T_{p_{2}} X_{2}\right)=T_{q} Y,
$$

for in this case the set-theoretic fiber product

$$
\begin{gathered}
X_{1} \times_{Y} X_{2}=\left\{\left(p_{1}, p_{2}\right) ; f_{1}\left(p_{1}\right)=f_{2}\left(p_{2}\right)\right\} \subset X_{1} \times X_{2}, \\
h_{i}\left(p_{1}, p_{2}\right)=p_{i}
\end{gathered}
$$

is a smooth manifold and the maps afforded by the universal property of fiber products are smooth.

The natural analog, 'b-transversality', of (I.5) in the setting of manifolds with corners is the requirement that

$$
{ }^{\mathrm{b}}\left(f_{1}\right)_{*}\left({ }^{\mathrm{b}} T_{p_{1}} X_{1}\right)+{ }^{\mathrm{b}}\left(f_{2}\right)_{*}\left({ }^{\mathrm{b}} T_{p_{2}} X_{2}\right)={ }^{\mathrm{b}} T_{q} Y .
$$

Under this condition, (I.6) is not necessarily a manifold, but can be decomposed as a union of what are here called 'interior binomial subvarieties'. These are objects generalizing manifolds with corners, with smooth interiors but mild singularities at the boundary and boundary faces which are of the same type. They can be resolved, by generalized boundary blow-up, to manifolds with corners.

As for a manifold, there is a natural monoidal complex $\mathcal{P}_{D}$ defined over the boundary faces of an interior binomial subvariety $D \subset X$, the difference being that the monoids may not be freely generated, i.e. may not be smooth. If the monoidal complex is smooth, then $D$ has a natural structure of a smooth manifold, although this may not be induced from $X$, in that $D$ may not be embedded. Even if the complex is not smooth, there is a smooth manifold $[D ; \mathcal{R}] \longrightarrow D$ corresponding to every smooth refinement $\mathcal{R} \longrightarrow \mathcal{P}_{D}$. 
In the case of fiber products, the monoids in the monoidal complex over $X_{1} \times_{Y} X_{2}$ are of the form

$$
\sigma_{F_{1}} \times_{\sigma_{G}} \sigma_{F_{2}}, \quad F_{i} \in \mathcal{M}\left(X_{i}\right),
$$

where $G=\left(f_{1}\right)_{\#}\left(F_{1}\right) \cap\left(f_{2}\right)_{\#}\left(F_{2}\right) \in \mathcal{M}(Y)$.

Theorem B. If $f_{i}: X_{i} \longrightarrow Y$ are b-normal b-maps which satisfy (I.7), if $X_{1} \times_{Y}$ $\stackrel{\circ}{X}_{2} \neq \emptyset$, and if each of the monoids (I.8) is freely generated, then there exists a natural smooth structure on (I.6) with respect to which it is the universal fiber product of $f_{1}$ and $f_{2}$ in the category of manifolds with corners.

This result extends a theorem of Joyce Joy09 on fiber products of more restricted maps. The condition that $\stackrel{\circ}{X}_{1} \times_{Y} \stackrel{\circ}{X}_{2} \neq \emptyset$ can be weakened: see Theorem 11.6 .

In the general case, when the monoids are not necessarily smooth, there is no such universal object and it is in general necessary to blow up to get smoothness and also to factor maps.

Theorem C. For every smooth refinement $\mathcal{R}$ of the complex $\mathcal{P}_{X_{1} \times_{Y} X_{2}}$, there is a manifold $\left[X_{1} \times_{Y} X_{2} ; \mathcal{R}\right]$ with b-maps to $X_{i}$ commuting with the $f_{i}: X_{i} \longrightarrow Y$ and such that if $g_{i}: Z \longrightarrow X_{i}, i=1,2$, are b-maps commuting with the $f_{i}$, then there exists a generalized blow-up $[Z ; \mathcal{S}] \longrightarrow Z$ and a canonical map $g:[Z ; \mathcal{S}] \longrightarrow$ $\left[X_{1} \times_{Y} X_{2} ; \mathcal{R}\right]$ giving a commutative diagram

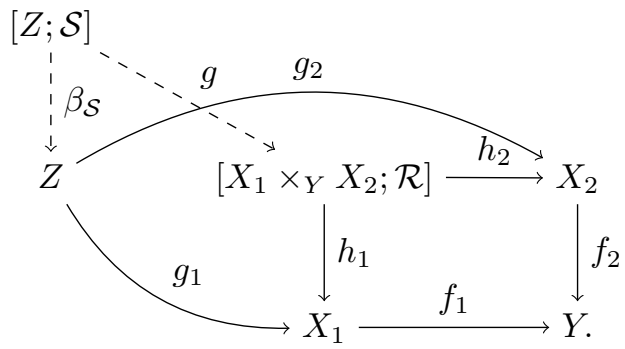

There is a close relationship between the material discussed here and the theory of logarithmic structures in algebraic geometry. The monoidal complexes we describe are related to 'fans' as defined by Kato [Kat94], which essentially are to (toric) monoids what schemes are to rings. Our generalized blow-up is then related to a result of Kato, which produces a resolution of a logarithmic scheme with 'toric singularities' (also called a 'log-smooth scheme') from a subdivision of the fan associated to the scheme. Our manifolds with corners and binomial subvarieties can be viewed as log smooth spaces, albeit with a stronger differentiable structure than is usually considered in algebraic geometry. There is however an important freedom afforded by working over $\mathbb{R}_{+}$(as is the case for manifolds with corners and boundary blow-up) rather than $\mathbb{R}$ or $\mathbb{C}$ which is that affine charts may be glued together by maps involving radicals and still result in smooth objects (as opposed to quite singular objects like stacks in the more conventional toric settings). We make heavy use of this fact and note that many of the smooth maps we produce would not even be birational in an algebraic setting over $\mathbb{C}$.

In Section 1 we briefly recall the theory of manifolds with corners and b-maps, and establish some notation. Section 2 contains a discussion of monoids (here 
always meaning what are often called 'toric monoids') and their refinements, which is enough to establish the local version of generalized blow-up in Section 3 . In Sections 4 and 5 we discuss the theory of monoidal complexes and the refinements of these. In Section 6 we complete one half of Theorem A, showing the existence of a generalized blow-up of $X$ given a smooth refinement of $\mathcal{P}_{X}$, along with the lifting results for b-maps, before exhibiting ordinary boundary blow-up and its iterated version as special cases in Section 7 . Section 8 completes Theorem A, giving the characterization of generalized blow-down maps by the properties (I.3) and (I.4). In Sections 9 and 10 we discuss the theory of interior binomial varieties and their resolution, finally applying this to fiber products in Section 11.

\section{MANifOldS With CORNERS}

In this section, we fix notation used for manifolds with corners and b-maps. For background, see [Mel] and Mel92. Set

$$
\mathbb{R}_{+}=[0, \infty) \text { and } \mathbb{N}=\{0,1,2, \ldots\} .
$$

The model manifold with corners is a product

$$
\mathbb{R}^{n, k}=\mathbb{R}_{+}^{k} \times \mathbb{R}^{n-k},
$$

for $k \in\{0, \ldots, n\}$, on which the smooth functions, forming the $\operatorname{ring} C^{\infty}\left(\mathbb{R}^{n, k}\right)=$ $\left.C^{\infty}\left(\mathbb{R}^{n}\right)\right|_{\mathbb{R}^{n, k}}$, are taken to be those obtained by restriction from the smooth functions on $\mathbb{R}^{n}$.

In general, a manifold with corners $X$ is a (paracompact, Hausdorff) topological manifold with boundary, $X$, with a ring of smooth functions, $C^{\infty}(X)$, with respect to which it is everywhere locally diffeomorphic to one of these model spaces. Thus, $X$ has a covering by coordinate patches with homeomorphisms to open subsets of the local model spaces such that $u \in C^{\infty}(X)$ if and only if its image in each coordinate system is smooth on the model. The pull-backs of the functions on the model spaces give local coordinate systems.

Each point $p \in X$ necessarily has a well-defined (boundary) codimension given by the number of independent non-negative coordinate functions vanishing at $p$ in such a coordinate system. A boundary face of codimension $k$ is the closure of one of the components of the set of points of codimension $k$; the set of such faces is denoted $\mathcal{M}_{k}(X)$. In particular, $\mathcal{M}_{1}(X)$ consists of boundary hypersurfaces and $\mathcal{M}_{0}(X)=X$ (or the set of components of $X$ if it is not connected). We set $\mathcal{M}(X)=\bigcup_{k} \mathcal{M}_{k}(X)$; this is a partially ordered set under inclusion.

As part of the definition of a manifold with corners, we require that all boundary hypersurfaces $H \in \mathcal{M}_{1}(X)$ be embedded; this is equivalent to insisting that the ideal of smooth functions vanishing on $H, \mathcal{I}_{H} \subset C^{\infty}(X)$, is principal. A nonnegative generator $\rho_{H} \in C^{\infty}(X)$ of this ideal is a defining function, so $\mathcal{I}_{H}=$ $\rho_{H} \cdot C^{\infty}(X)$. It follows that each boundary face of $X$ is itself a manifold with corners. While it would be possible to drop this requirement of embedded hypersurfaces and still retain many of the results in this paper, manifolds violating this property tend to be very ill-behaved from an analytical standpoint, and often the first step when encountering such a space is to resolve it via boundary blow-up to a space with embedded hypersurfaces. In light of this, the simplification in the combinatorial description of boundary faces which results from the stronger requirement is a worthy trade-off. 
The diffeomorphisms of $X$, homeomorphisms which map $C^{\infty}(X)$ to itself, must preserve the stratification by boundary codimension, and the infinitesimal diffeomorphisms correspond to the Lie subalgebra

$$
\mathcal{V}_{\mathrm{b}}(X) \subset \mathcal{V}(X)
$$

of the usual algebra of real smooth vector fields, consisting of those vector fields which are tangent to each of the boundary faces of $X$. This subalgebra is the space of global smooth sections of the b-tangent bundle, ${ }^{\mathrm{b}} T X \longrightarrow X$. Locally, whenever $(x, y): U \longrightarrow \mathbb{R}_{+}^{k} \times \mathbb{R}^{n-k}$ are coordinates centered at $p$,

$$
{ }^{\mathrm{b}} T_{p} X=\operatorname{span}_{\mathbb{R}}\left\{x_{1} \partial_{x_{1}}, \ldots, x_{k} \partial_{x_{k}}, \partial_{y_{k+1}}, \ldots, \partial_{y_{n}}\right\} .
$$

From (1.1), there is a natural evaluation map

$$
{ }^{\mathrm{b}} T X \longrightarrow T X
$$

which is an isomorphism over the interior of $X$ but not over the boundary. Over interior points $p \in \stackrel{\circ}{F} \in \mathcal{M}_{k}(X)$ (and extending to all of $F$ by continuity), the kernel of (1.2) defines the b-normal bundle:

$$
{ }^{\mathrm{b}} N F \longrightarrow F,{ }^{\mathrm{b}} N_{p} F=\operatorname{span}_{\mathbb{R}}\left\{x_{1} \partial_{x_{1}}, \ldots, x_{k} \partial_{k_{k}}\right\} \text {, locally. }
$$

In fact, up to reordering, the sections $x_{i} \partial_{x_{i}}, i=1, \ldots, k$, of ${ }^{\mathrm{b}} N F$ are well defined independent of coordinates since any other set $\left(x_{1}^{\prime}, \ldots, x_{k}^{\prime}\right)$ must have the form (reordering if necessary) $x_{i}^{\prime}=a_{i}(x, y) x_{i}$ with $a_{i}>0$, so

$$
x_{i}^{\prime} \partial_{x_{i}^{\prime}}=x_{i} \partial_{x_{i}}+\mathcal{O}(x) \mathcal{V}_{\mathrm{b}}(X),
$$

and hence $x_{i}^{\prime} \partial_{x_{i}^{\prime}} \equiv x_{i} \partial_{x_{i}}$ at $F$.

Thus, ${ }^{\mathrm{b}} N F \longrightarrow F$ is canonically trivial, with a well-defined lattice

$$
\left({ }^{\mathrm{b}} N F\right)_{\mathbb{Z}}=\operatorname{span}_{\mathbb{Z}}\left\{x_{1} \partial_{x_{1}}, \ldots, x_{k} \partial_{x_{k}}\right\}
$$

and cone of inward-pointing vectors

$$
{ }^{\mathrm{b}} N_{+} F=\operatorname{span}_{\mathbb{R}_{+}}\left\{x_{1} \partial_{x_{1}}, \ldots, x_{k} \partial_{x_{k}}\right\} .
$$

These play a fundamental role in the discussion here.

A b-map $f: X \longrightarrow Y$ between manifolds with corners is a map which is smooth, meaning $f^{*} C^{\infty}(Y) \subset C^{\infty}(X)$, and is such that $f$ pulls back each (principal) ideal $\mathcal{I}_{H}$ to either a product of powers of such ideals on $X$,

$$
f^{*} \mathcal{I}_{H}=\prod_{G \in \mathcal{M}_{1}(X)} \mathcal{I}_{G}^{\alpha(G, H)}, \alpha(G, H) \in \mathbb{N}
$$

to the zero ideal, $f^{*} \mathcal{I}_{H}=0$ or to $C^{\infty}(X)$. If the second case does not occur we say $f$ is an interior b-map. Otherwise $f: X \longrightarrow F$ is an interior b-map for some $F \in \mathcal{M}(Y)$. We shall mostly be concerned with interior b-maps in this paper. In practical terms (1.4) means that $f$ has the local form

$$
\begin{gathered}
f: \mathbb{R}_{+}^{n} \times \mathbb{R}^{k} \ni(x, y) \longmapsto\left(x^{\prime}, y^{\prime}\right) \in \mathbb{R}_{+}^{n^{\prime}} \times \mathbb{R}^{k^{\prime}}, \\
\left(x_{1}^{\prime}, \ldots, x_{n^{\prime}}^{\prime}\right)=\left(a_{1}(x, y) \prod_{i} x_{i}^{\alpha(i, 1)}, \ldots, a_{n^{\prime}}(x, y) \prod_{i} x^{\alpha\left(i, n^{\prime}\right)}\right), \\
y^{\prime}=b(x, y),
\end{gathered}
$$


where the $a_{i}(x, y)$ are strictly positive smooth functions and $\alpha(i, j)=\alpha(G, H)$, where $G$ and $H$ are the boundary hypersurfaces for which $x_{i}$ and $x_{j}^{\prime}$ are locally defining functions, respectively.

For an interior b-map the differential $f_{*}: T X \longrightarrow T Y$ extends by continuity from the interior to the $b$-differential

$$
{ }^{\mathrm{b}} f_{*}:{ }^{\mathrm{b}} T_{p} X \longrightarrow{ }^{\mathrm{b}} T_{f(p)} Y, \forall p \in X .
$$

We denote by $f_{\#}: \mathcal{M}(X) \longrightarrow \mathcal{M}(Y)$ the map which assigns to each boundary face of $X$ the boundary face of $Y$ of largest codimension which contains it. Then the differential restricts to a map

$$
{ }^{\mathrm{b}} f_{*}:{ }^{\mathrm{b}} N F \longrightarrow{ }^{\mathrm{b}} N f_{\#}(F)
$$

which is integral with respect to the lattices (1.3). Indeed, (1.5) is given by a matrix with integer entries coming from the exponents $\alpha(G, H)$ in (1.4), and ${ }^{\mathrm{b}} f_{*}$ maps inward-pointing vectors to inward-pointing vectors since these entries are non-negative.

It is convenient to use multi-index notation for certain maps in coordinates. If $t=\left(t_{1}, \ldots, t_{n}\right)$ and $x=\left(x_{1}, \ldots, x_{k}\right)$ are local coordinates on spaces $U$ and $V$ respectively, and $\mu \in \operatorname{Mat}(n \times k, \mathbb{R})$ is a matrix, the map $\left(t_{1}, \ldots, t_{n}\right) \longmapsto\left(x_{1}, \ldots, x_{n}\right)$, where

$$
x_{i}=\prod_{j} t_{j}^{\mu_{j i}} \text { is denoted } t \longmapsto t^{\mu}=x .
$$

With this convention on the order of the indices, $\left(t^{\mu}\right)^{\nu}=t^{\mu \nu}$.

\section{Monoids}

In general terms a monoid is a set which is closed under an associative binary operation (usually required to be commutative and have an identity element), so in essence a group without inverses or a ring without addition. Here we restrict attention to monoids of the special type usually known as toric monoids, which for our purposes may be characterized as follows.

Definition 2.1. A toric monoid is a set $\sigma$, closed under a binary operation of addition, which can be expressed as the intersection

$$
\sigma=N_{\sigma} \cap C
$$

of a finitely generated integral lattice $N_{\sigma}$ and a proper convex polyhedral cone $C$ in the vector space $N_{\sigma}^{\mathbb{R}}=N_{\sigma} \otimes_{\mathbb{Z}} \mathbb{R}$ which is integral with respect to $N_{\sigma}$, and for which $N_{\sigma}^{\mathbb{R}}=\operatorname{span}_{\mathbb{R}} \sigma$. The cone, which is determined from the monoid by $C=\operatorname{span}_{\mathbb{R}_{+}} \sigma \subset N_{\sigma}^{\mathbb{R}}$, will be called the support of $\sigma$ and denoted by

$$
\operatorname{supp}(\sigma)=C \subset N_{\sigma}^{\mathbb{R}} .
$$

The minimal integral generators $\left\{v_{1}, \ldots, v_{k}\right\} \subset N_{\sigma}$ of the cone $\operatorname{supp}(\sigma)$ (which do not necessarily generate $\sigma$ as a monoid) will be called the extremals of $\sigma$; by assumption no $v_{i}$ is a combination of the others with non-negative coefficients, but every point of $\operatorname{supp}(\sigma)$ is a non-negative linear combination of the $v_{i}$.

Toric monoids may be characterized equivalently (though more abstractly) as follows Ogu06. For $\sigma$ a commutative, finitely generated monoid with identity, there is a canonical abelian group $\sigma^{\mathrm{gp}}$ which is universal with respect to monoid homomorphisms from $\sigma$ to groups. Then $\sigma$ is a toric monoid provided it is sharp, 
meaning $\sigma$ has no invertible elements besides 0 ; integral, meaning that cancellation holds: if $x+y=z+y \in \sigma$, then $x=z \in \sigma$ (equivalently the map from $\sigma$ to $\sigma^{\mathrm{gp}}$ is injective); saturated, meaning that if $n v \in \sigma$ for some $n>0$ and $v \in \sigma^{\mathrm{gp}}$, then $v \in \sigma$; and torsion free, meaning that $\sigma^{\mathrm{gp}}$ is torsion free. Indeed, under these conditions $N_{\sigma}:=\sigma^{\text {gp }}$ is a lattice, $C=\operatorname{span}_{\mathbb{R}_{+}} \sigma \subset N_{\sigma} \otimes_{\mathbb{Z}} \mathbb{R}$ is a proper convex integral cone and $\sigma \equiv N_{\sigma} \cap C$.

For the remainder of the paper, monoid will always mean toric monoid.

The dimension $\operatorname{dim}(\sigma)$ is the dimension of $N_{\sigma}^{\mathbb{R}}$; equivalently, $\operatorname{dim}(\sigma)$ is the maximum number of linearly independent extremals. A monoid $\sigma$ is said to be simplicial if the extremals $\left\{v_{1}, \ldots, v_{n}\right\}$ are independent, in which case $\operatorname{dim}(\sigma)=n$ and $\operatorname{supp}(\sigma)$ is a cone over the $n-1$ simplex defined by $\left\{v_{1}, \ldots, v_{n}\right\}$. A simplicial monoid is further said to be smooth if it is generated as a monoid by its extremals. A smooth monoid is therefore freely generated and isomorphic to $\mathbb{N}^{n}$. We will use the notation $\sigma=\mathbb{N}\left\langle v_{1}, \ldots, v_{n}\right\rangle$ to denote a smooth monoid freely generated by independent vectors $v_{1}, \ldots, v_{n}$.

One monoid $\sigma^{\prime}$ in $N$ is a submonoid of another $\sigma$ if $\sigma^{\prime} \subset \sigma$, so the generators of $\sigma^{\prime}$ are non-negative integral combinations of the generators of $\sigma$.

A monoid $\tau$ is a face of $\sigma$, written $\tau \leq \sigma$, if $\tau$ is a submonoid such that whenever $v, w \in \sigma$ and $v+w \in \tau$, then $v, w \in \tau$. Equivalently, $\tau$ is the largest submonoid contained in a face (in the sense of cones) of $\operatorname{supp}(\sigma)$, which is in turn equivalent to the existence of a functional $u \in N_{\sigma}^{*}$ such that

$$
\begin{array}{ll}
\langle u, v\rangle=0 & \text { for } v \in \tau, \\
\langle u, v\rangle>0 & \text { for } v \in \sigma \backslash \tau .
\end{array}
$$

In particular, the trivial monoid $\{0\}$ with no generators is a face of every monoid.

A monoid homomorphism $\phi: \sigma^{\prime} \longrightarrow \sigma$ is just an addition preserving map. We will also denote by $\phi$ the induced linear maps $\phi: N_{\sigma^{\prime}} \longrightarrow N_{\sigma}, \phi: N_{\sigma^{\prime}}^{\mathbb{R}} \longrightarrow N_{\sigma}^{\mathbb{R}}$ and $\phi: \operatorname{supp}\left(\sigma^{\prime}\right) \longrightarrow \operatorname{supp}(\sigma)$. Note that unless $\phi$ is injective, the image of $\sigma^{\prime}$ in $\sigma$ need not be a monoid in the sense we have defined, since it may not be saturated (consider the map $\mathbb{N}^{2} \longrightarrow \mathbb{N}$ sending $(1,0)$ to 2 and $(0,1)$ to 3 , for instance). The inverse image of a face of $\sigma$ is always a face of $\sigma^{\prime}$.

Definition 2.2. Given a monoid $\sigma$, a refinement of $\sigma$ is a collection $R$ of submonoids of $\sigma$, such that

(i) if $\sigma^{\prime} \in R$ and $\tau^{\prime} \leq \sigma^{\prime}$, then $\tau^{\prime} \in R$,

(ii) for any two monoids $\sigma_{1}^{\prime}, \sigma_{2}^{\prime} \in R, \sigma_{1}^{\prime} \cap \sigma_{2}^{\prime}$ must be a face of both $\sigma_{1}^{\prime}$ and $\sigma_{2}^{\prime}$, and

(iii) $\bigcup_{i} \operatorname{supp}\left(\sigma_{i}\right)=\operatorname{supp}(\sigma)$ (as viewed in the vector space $N_{\sigma}^{\mathbb{R}}$ using the natural inclusions $\left.N_{\sigma_{i}}^{\mathbb{R}} \longrightarrow N_{\sigma}^{\mathbb{R}}\right)$.

We say $R$ is a simplicial (resp. smooth) refinement if each $\sigma_{i} \in R$ is simplicial (resp. smooth). The set of faces of $\sigma$ (including $\sigma$ itself) form the trivial refinement of $\sigma$.

These conditions imply that the cones $\operatorname{supp}\left(\sigma_{i}\right)$ form a fan - in the classical sense used in the theory of toric varieties [Ful93] - whose support is equal to that of $\sigma$, though it is important to note that this notion of refinement differs from the standard one in toric geometry in that the monoids need not all be defined with respect to a single lattice. In particular, the 'Fulton-style' fan refinement underlying a smooth refinement need not be smooth.

A refinement of $\sigma$ is a special case of a monoidal complex, discussed in Section 4 , 

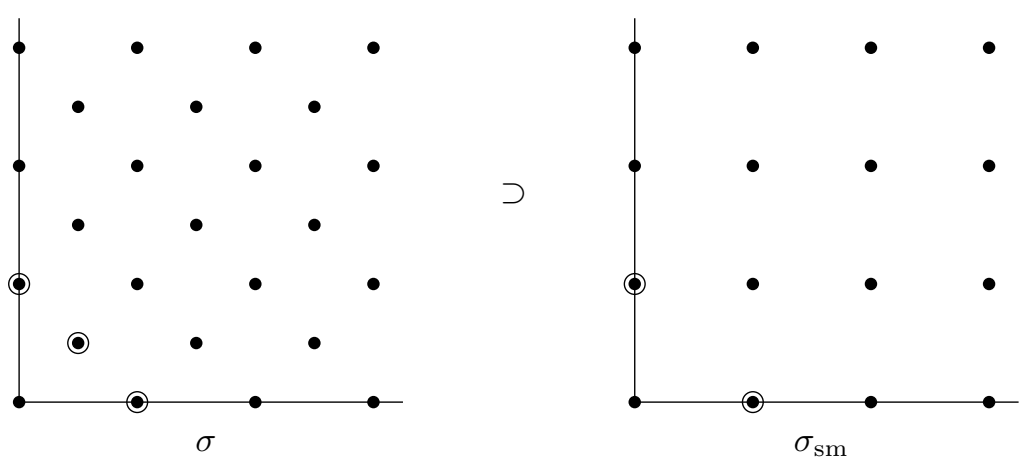

FiguRE 1. A non-smooth, simplicial monoid $\sigma$, along with its smoothing $\sigma_{\mathrm{sm}}$. Generators of each monoid are highlighted.

Example 2.3. A simplicial monoid, $\sigma$, with extremals $\left\{v_{1}, \ldots, v_{n}\right\}$, has a canonical smooth refinement consisting of the faces of the smooth monoid

$$
\sigma_{\mathrm{sm}}:=\mathbb{N}\left\langle v_{1}, \ldots, v_{n}\right\rangle .
$$

These are of the form $\mathbb{N}\left\langle w_{1}, \ldots, w_{k}\right\rangle$ for all subsets $\left\{w_{1}, \ldots, w_{k}\right\} \subseteq\left\{v_{1}, \ldots, v_{n}\right\}$; this refinement will be called the smoothing of $\sigma$. See Figure 1 .

This example illustrates the point that while $\operatorname{supp}(\sigma)$ is covered by supports of the $\tau \in R, \sigma$ need not be the union of the $\tau \in R$ as a monoid. A simpler example of this phenomenon is the refinement of $\mathbb{N}$ given by the submonoid $2 \mathbb{N}=\{0,2,4, \ldots\}$.

It is important to observe that this smoothing operation is rather non-standard; in the theory of complex toric varieties for instance, it may generate a map of varieties which is not even birational. However, in the category of manifolds with corners (where ' $\mathbb{R}_{+}$-points' are considered and radicals are consequently well behaved) it becomes very useful and will be fundamental to our results below.

Lemma 2.4. If $R$ is a refinement of $\sigma$ and $\tau \leq \sigma$ is a face, then

$$
R(\tau)=\left\{\sigma^{\prime} \in R ; \sigma^{\prime} \subset \tau\right\}
$$

is a refinement of $\tau$.

Proof. Clearly $R(\tau)$ satisfies the first two properties of a refinement, since if $\sigma^{\prime} \in$ $R(\tau)$ and $\tau^{\prime} \leq \sigma^{\prime}$, then $\tau^{\prime} \subset \tau$ and hence $\tau^{\prime} \in R(\tau)$. For the third property, choose any $v \in \operatorname{supp}(\tau)$. Since also $v \in \operatorname{supp}(\sigma)$, there must be a $\sigma^{\prime} \in R$ for which $v \in \operatorname{supp}\left(\sigma^{\prime}\right)$. By the support condition, $\operatorname{supp}\left(\sigma^{\prime}\right) \cap \operatorname{supp}(\tau)$ is a face of $\operatorname{supp}\left(\sigma^{\prime}\right)$, which corresponds to a monoid $\tau^{\prime} \leq \sigma^{\prime}$ such that $\operatorname{supp}\left(\tau^{\prime}\right) \subset \operatorname{supp}(\tau)$.

A particularly important example of a refinement is the operation of star subdivision, which is well known in toric geometry [Ful93] and its generalizations, though the definition below is not standard. (In the classical toric theory, the subdivision of a smooth fan need not be smooth, for instance.)

Proposition 2.5. If $\sigma$ is a monoid and $0 \neq v \in \sigma$, the collection, $S(\sigma, v)$, of monoids consisting of

(i) all faces $\tau \leq \sigma$ such that $v \notin \tau$ and

(ii) monoids $\tau+\mathbb{N} v:=\operatorname{span}_{\mathbb{N}} \tau \cup\{v\}$, where $\tau \leq \sigma$ and $v \notin \tau$ 
is a refinement of $\sigma$. If all proper faces of $\sigma$ are smooth, then $S(\sigma, v)$ is a smooth refinement.

Proof. For two monoids $\tau_{1}, \tau_{2}$ of the first type, the intersection $\tau_{1} \cap \tau_{2}$ is a face of $\sigma$ not containing $v$, hence a monoid of the first type. For two monoids $\tau_{1}+\mathbb{N} v$ and $\tau_{2}+\mathbb{N} v$ of the second type, the intersection is a monoid $\left(\tau_{1} \cap \tau_{2}\right)+\mathbb{N} v$ of the second type, and for one of each type, the intersection $\left(\tau_{1}+\mathbb{N} v\right) \cap \tau_{2}=\tau_{1} \cap \tau_{2}$ is of the first type. The support condition follows from the fact that any $w \in \operatorname{supp}(\sigma)$ lies in some cone of the form $\operatorname{supp}(\tau+\mathbb{N} v)$, with $\tau \leq \sigma$.

If $\tau=\mathbb{N}\left\langle v_{1}, \ldots, v_{k}\right\rangle$ is smooth and $v \notin \tau$, then $\tau+\mathbb{N} v$ has independent generators $\left\{v_{1}, \ldots, v_{k}, v\right\}$, so $\tau+\mathbb{N} v=\mathbb{N}\left\langle v_{1}, \ldots, v_{k}, v\right\rangle$ is smooth. Since $\sigma$ itself is not in $S(\sigma, v)$, smoothness follows from the smoothness of all the proper faces of $\sigma$.

In the context of a generalized blow-up, star subdivision is the operation which realizes the ordinary blow-up of a boundary face of a manifold. There is a similar construction, "planar refinement", which we discuss next although it is not used until Section 10 in which $v$ is replaced by the intersection of $\sigma$ with a subspace.

First, as a matter of notation, suppose $\tau_{i} \subset \sigma, i=1,2$, are full submonoids in the sense that each

$$
\tau_{i}=\sigma \cap \operatorname{supp}\left(\tau_{i}\right) \text {. }
$$

Then we define the join of $\tau_{1}$ and $\tau_{2}$ to be the full submonoid

$$
\tau_{1} * \tau_{2}:=\sigma \cap\left(\operatorname{supp}\left(\tau_{1}\right)+\operatorname{supp}\left(\tau_{2}\right)\right),
$$

where $\operatorname{supp}\left(\tau_{1}\right)+\operatorname{supp}\left(\tau_{2}\right)=\operatorname{span}_{\mathbb{R}_{+}}\left(\tau_{1} \cup \tau_{2}\right)$ is the convex hull of the supports of the $\tau_{i}$.

Let $\sigma$ be a smooth monoid and $\mu \subset \sigma$ be a submonoid which is obtained by intersection with an integral subspace of $N_{\sigma}^{\mathbb{R}}$ :

$$
\mu=\sigma \cap M, \quad M \subset N_{\sigma}^{\mathbb{R}} \text { integral. }
$$

Note that $\mu$ need not be simplicial, though it is a full submonoid in the sense of (2.2). Let $\Lambda$ consist of the maximal faces of $\sigma$ meeting $\mu$ trivially:

$$
\Lambda=\left\{\tau \leq \sigma ; \tau \cap \mu=\{0\}, \text { but for all } \tau^{\prime}>\tau, \tau^{\prime} \cap \mu \neq\{0\}\right\} .
$$

Lemma 2.6. If $\sigma$ is a smooth monoid, $M$ is an integral subspace and $\mu$ and $\Lambda$ are defined by (2.4) and (2.5), then for any $\tau_{1} \neq \tau_{2} \in \Lambda$, there exists a functional $u \in N_{\sigma}^{*}$ such that $\left\langle u, \tau_{1}\right\rangle \geq 0,\left\langle u, \tau_{2}\right\rangle \leq 0,\langle u, \mu\rangle=0$, and if $v$ is a generator of either $\tau_{1}$ or $\tau_{2}$, then $v \in \tau_{1} \cap \tau_{2}$ if and only if $\langle u, v\rangle=0$.

Proof. If $M$ has full dimension the result is trivial, so we may assume that $M$ is a proper subspace. If $M$ is a hyperplane, then $u$ is a defining functional, $u^{\perp}=M$, and is determined up to non-vanishing constant. Since $\tau_{i} \cap M=\{0\}, u$ is definite on each and cannot have the same sign on both or they would be equal by maximality. Thus, either $\left\langle u, \tau_{1}\right\rangle>0,\left\langle u, \tau_{2}\right\rangle<0$ or with signs reversed. In this case their intersection is trivial and the result clearly holds. Conversely if $\tau_{1} \cap \tau_{2} \neq\{0\}$, then $\operatorname{codim}(M)>1$.

In the general case we proceed by induction on $\operatorname{codim}(M)$. Choose a generator $w \in \tau_{1} \cap \tau_{2}$, and consider the smooth monoid $\sigma^{\prime}<\sigma$ generated by all the generators of $\sigma$ except $w$, with $\tau_{i}^{\prime}:=\tau_{i} \cap \sigma^{\prime}$. Consider the subspace $M^{\prime}=(M+\mathbb{R} w) \cap N_{\sigma^{\prime}} \subset N_{\sigma^{\prime}}$ and set $\mu^{\prime}=M^{\prime} \cap \sigma^{\prime}$. Since $w \notin M, M^{\prime}$ has the same dimension as $M$; hence the codimension of $M^{\prime}$ in $N_{\sigma^{\prime}}$ is one less than the codimension of $M$ in $N_{\sigma}$. 
Now, the $\tau_{i}^{\prime}$ are maximal faces of $\sigma^{\prime}$ not meeting $\mu^{\prime}$. To see this first note that $\tau_{i}^{\prime} \cap M^{\prime}=\{0\}$ since the generators of $\mu$ can be written $e_{l}=e_{l}^{\prime}+c_{l} w$, where the $e_{l}^{\prime}$ are generators for $\mu^{\prime}$ and $c_{l} \geq 0$. Thus, if there was a point $p \in \tau_{i}^{\prime} \cap \mu^{\prime}$, necessarily of the form $p=\sum_{l} d_{l} e_{l}^{\prime}$ with the $d_{l} \geq 0$, then there would be a point $p+\sum_{l} d_{l} c_{l} w \in$ $\tau_{i} \cap \mu$. Similarly, the $\tau_{i}^{\prime}$ are maximal since if $\tau^{\prime}>\tau_{i}^{\prime}$ is a face of $\sigma^{\prime}$ which meets $\mu^{\prime}$ trivially then $\tau^{\prime}+\mathbb{N} w>\tau_{i}$ meets $\mu$ trivially, contradicting the maximality of $\tau_{i}$.

Thus by induction on the codimension of $M^{\prime}$ in $N_{\sigma^{\prime}}$ there exists a functional $u^{\prime} \in N_{\sigma^{\prime}}^{*}$ with the desired separating property for the $\tau_{i}^{\prime}$ and $M^{\prime}$. Extending $u^{\prime}$ to $u$ by requiring $u(w)=0$ gives a functional in $N_{\sigma}^{*}$ with the desired properties and completes the inductive step.

Now set $\mu * \Lambda=\{\mu * \tau ; \tau \in \Lambda\}$, defined by (2.3), and consider

$$
S(\sigma, \mu)=\left\{\gamma \leq \sigma^{\prime} ; \sigma^{\prime} \in \mu * \Lambda\right\} .
$$

Proposition 2.7 (Planar refinement). If $\sigma$ is smooth monoid and $\mu=\sigma \cap M$ is the intersection with an integral subspace $M \subset N_{\sigma}^{\mathbb{R}}$, then (2.6) gives a refinement of $\sigma$ containing $\mu$. This operation commutes with faces in the sense that for any face $\tau \leq \sigma, S(\tau, \tau \cap \mu)=(S(\sigma, \mu))(\tau)$ with notation as in (2.1).

Proof. If $M=N_{\sigma}^{\mathbb{R}}$, then $\mu=\sigma, \Lambda=\{0\}$ and $\mathcal{S}(\sigma, \mu)=\mu *\{0\}$ consists of $\sigma$ and its faces, which is the trivial refinement of $\sigma$.

First we show that the supports of $S(\sigma, \mu)$ cover $\operatorname{supp}(\sigma)$; in fact,

$$
\sigma=\bigcup_{\tau \in \Lambda} \mu * \tau
$$

since all the monoids are full as above. Certainly $\mu \subset \tau * \mu$, so consider $v \in \sigma \backslash \mu$ and choose any $m_{1} \in \mu$. The ray from $m_{1}$ through $v$ meets some face of $\sigma$, call it $\tau_{1}$, so $v$ is a positive combination of $m_{1} \in \mu$ and $t_{1} \in \tau_{1}$. If $\tau_{1} \cap \mu=\{0\}$, then $\tau_{1} \subset \tau \in \Lambda$ and we are finished. On the other hand, if $\tau_{1}$ meets $\mu$, then $t_{1}$ is a positive combination of some $m_{2} \in \mu$ and $t_{2} \in \tau_{2}$ with $\tau_{2}$ a proper face of $\tau_{1}$. Continuing this way eventually shows that $v \in \mu * \tau_{k}$ for some $\tau_{k}$ which does not meet $\mu$, since the dimension decreases and $v \notin \mu$. Thus $v \in \mu * \tau$ for some $\tau \in \Lambda$ with $\tau \geq \tau_{k}$, and (2.7) follows.

Next consider the intersection of two elements in the union (2.7). We will show that

$$
\left(\mu * \tau_{1}\right) \cap\left(\mu * \tau_{2}\right)=\mu *\left(\tau_{1} \cap \tau_{2}\right), \tau_{i} \in \Lambda .
$$

Certainly the right side is contained in the left. Lemma 2.6 applies and gives a functional $u$. If $v$ is in the intersection it follows that $u(v) \geq 0$ and $u(v) \leq 0$, so $u(v)=0$ and from the last property of $u$ this implies $v \in \mu *\left(\tau_{1} \cap \tau_{2}\right)$. Since $u$ is a supporting hyperplane for both $\mu * \tau_{i}$, it follows that $\mu *\left(\tau_{1} \cap \tau_{2}\right)$ is a boundary face of each of these full submonoids. This in turn implies that the intersection of any two elements of $S(\sigma, \mu)$ is a common boundary face of both and hence an element of $S(\sigma, \mu)$. Indeed, such an intersection must be contained in two $\mu * \tau_{i}$ with $\tau_{i} \in \Lambda$. If they are the same, the conclusion is immediate, and if not, then they are both contained in the common boundary face (2.8) and again the result follows.

That $\mu$ is a boundary face of each $\mu * \tau, \tau \in \Lambda$ follows from the existence of a functional $u \in N_{\sigma}^{*}$ such that $u>0$ on the generators of $\tau$ and $u(M)=0$. 
For any $v \in \sigma$, if $\mathbb{N} v$ is a full submonoid, then $S(\sigma, \mathbb{N} v)$ is just the ordinary star subdivision of $\sigma$ along $v$. Note however that in the case of star subdivision we can relax the condition that $\sigma$ be smooth, as well as the condition that $\mathbb{N} v$ be full.

\section{Generalized BlOW-UP of $\mathbb{R}_{+}^{n}$}

In preparation for the global case of generalized boundary blow-up of a manifold with corners treated in Section 6 we first discuss the 'local case' of generalized blowup of the model space $\mathbb{R}_{+}^{n}$. Associated with this basic space is the 'basic monoid' which is freely generated by the boundary hypersurfaces of $\mathbb{R}_{+}^{n}$, with an equivalent realization as the non-negative integral points in ${ }^{\mathrm{b}} N\{0\}$, the b-normal to the maximum codimension boundary face $\{0\}$. We show how to construct a blown-up space mapping surjectively onto $\mathbb{R}_{+}^{n}$ corresponding to any smooth refinement of this basic monoid. The functoriality of this operation with respect to diffeomorphisms and b-maps is then considered.

Consider $\mathbb{R}_{+}^{n}$ with coordinates $\left(x_{1}, \ldots, x_{n}\right)$ and let $H_{i}=\left\{x_{i}=0\right\} \in \mathcal{M}_{1}\left(\mathbb{R}_{+}^{n}\right)$ denote the boundary hypersurfaces. The basic monoid of $\mathbb{R}_{+}^{n}$ is the smooth monoid freely generated by the $H_{i}$ :

$$
\sigma_{\mathbb{R}_{+}^{n}}=\mathbb{N}\left\langle H_{1}, \cdots, H_{n}\right\rangle .
$$

There is a natural embedding of $\sigma_{\mathbb{R}_{+}^{n}}$ into the vector space

$$
{ }^{\mathrm{b}} N\{0\}=\operatorname{span}_{\mathbb{R}}\left\{x_{1} \partial_{x_{1}}, \ldots, x_{n} \partial_{x_{n}}\right\} .
$$

Indeed, as pointed out in Section 1, the vectors $\left(x_{1} \partial_{x_{1}}, \ldots, x_{n} \partial_{x_{n}}\right)$ are invariantly defined up to reordering with respect to diffeomorphisms (indeed, any diffeomorphism of $\mathbb{R}_{+}^{n}$ to itself must take 0 to 0 ), so $\sigma_{\mathbb{R}_{+}^{n}}$ may be identified with the inward pointing lattice points

$$
\sigma_{\mathbb{R}_{+}^{n}} \cong \mathbb{N}\left\langle x_{1} \partial_{x_{1}}, \ldots, x_{n} \partial_{x_{n}}\right\rangle \text { in }{ }^{\mathrm{b}} N\{0\} .
$$

From now on we will identify $\sigma_{\mathbb{R}_{+}^{n}}$ with this image in ${ }^{\mathrm{b}} N\{0\}$.

To any smooth refinement $R$ of $\sigma_{\mathbb{R}_{+}^{n}}$ we proceed to associate a generalized blow-up of $\mathbb{R}_{+}^{n}$, which will be denoted, with its blow-down map,

$$
\left[\mathbb{R}_{+}^{n} ; R\right] \stackrel{\beta}{\longrightarrow} \mathbb{R}_{+}^{n} .
$$

The blow-down map $\beta:\left[\mathbb{R}_{+}^{n} ; R\right] \longrightarrow \mathbb{R}_{+}^{n}$ is an interior b-map from this new manifold with corners, which is proper, surjective and restricts to a diffeomorphism on the interior of its domain to the interior of $\mathbb{R}_{+}^{n}$. We construct $\left[\mathbb{R}_{+}^{n} ; R\right]$ and $\beta$ from explicit coordinate patches and transition diffeomorphisms.

For brevity we will say that a monoid $\sigma \in R$ of maximal dimension $n$ is maximal. To any such maximal monoid $\sigma=\mathbb{N}\left\langle v_{1}, \ldots, v_{n}\right\rangle$, we associate a copy of the model space $U_{\sigma}=\mathbb{R}_{+}^{n}$ with coordinates $t=\left(t_{1}, \ldots, t_{n}\right)$, where the order of the coordinates is associated to the order of the vectors. Let $\nu \in \operatorname{GL}(n, \mathbb{Q})$ be the matrix whose rows are the coordinates of the vectors $v_{i}$ so that $\nu$ has entries $\nu_{i j}$, where

$$
v_{i}=\sum_{j=1}^{n} \nu_{i j} x_{j} \partial_{x_{j}}
$$

(using the realization of $\sigma_{\mathbb{R}_{+}^{n}}$ in ${ }^{\mathrm{b}} N\{0\}$ ). Since $R$ is a refinement of $\sigma_{\mathbb{R}_{+}^{n}}, \nu$ has non-negative integral entries:

$$
\nu \in \operatorname{Mat}(n \times n, \mathbb{N}) \cap \operatorname{GL}(n, \mathbb{Q}) .
$$


Then consider the smooth map

$$
\beta_{\sigma}: U_{\sigma} \ni t \longmapsto t^{\nu}=x \in \mathbb{R}_{+}^{n}
$$

where the image space is the fixed model manifold, and we use the notational convention established in Section 1. This is an interior b-map (which will be the local coordinate version of the blow-down map), which is a diffeomorphism of the interiors of its domain and range, under which

$$
\begin{aligned}
{ }^{\mathrm{b}}\left(\beta_{\sigma}\right)_{*}:{ }^{\mathrm{b}} N\{0\} \subset{ }^{\mathrm{b}} T_{0} U_{\sigma} \longrightarrow{ }^{\mathrm{b}} N\{0\} \subset{ }^{\mathrm{b}} T_{0} \mathbb{R}_{+}^{n}, \\
t_{i} \partial_{t_{i}} \longmapsto \sum_{j} \nu_{i j} x_{j} \partial_{x_{j}} \cong v_{i} .
\end{aligned}
$$

In addition, ${ }^{\mathrm{b}} \beta_{\sigma}:{ }^{\mathrm{b}} T_{p} U_{\sigma} \longrightarrow{ }^{\mathrm{b}} T_{\beta_{\sigma}(p)} \mathbb{R}_{+}^{n}$ is an isomorphism for all $p$.

To each face $\tau \leq \sigma$ given by a collection of generators, $\tau=\mathbb{N}\left\langle v_{i}\right\rangle_{i \in I}, I \subset$ $\{1, \ldots, n\}$, we associate the (relatively) open set

$$
U_{\sigma, \tau}=\left\{\left(t_{1}, \ldots, t_{n}\right) \in U_{\sigma} ; t_{j} \neq 0 \text { if } j \notin I\right\} \subset U_{\sigma} .
$$

Thus, $U_{\sigma, \tau} \cong \mathbb{R}_{+}^{k} \times(0, \infty)^{n-k} \subset \mathbb{R}_{+}^{n}$, where the coordinates allowed to take the value 0 are the $t_{i_{1}}, \ldots, t_{i_{k}}$, corresponding to those generators of $\sigma$ which are also generators of $\tau$.

Proposition 3.1 (Generalized blow-up of $\mathbb{R}_{+}^{n}$ ). For any two maximal monoids $\sigma_{1}$ and $\sigma_{2}$ in a smooth refinement $R$ of $\sigma_{\mathbb{R}_{+}^{n}}$, with common face $\tau=\sigma_{1} \cap \sigma_{2}$, the diffeomorphism of the interiors of the $U_{\sigma_{i}}$ given by $\beta_{\sigma_{2}}^{-1} \beta_{\sigma_{1}}$, extends by continuity to a diffeomorphism $\chi_{12}: U_{\sigma_{1}, \tau} \longrightarrow U_{\sigma_{2}, \tau}$ and the quotient space

$$
\left[\mathbb{R}_{+}^{n} ; R\right]=\left(\bigsqcup U_{\sigma}\right) /\left\{\chi_{*}\right\}
$$

is a manifold with corners, with coordinate charts the $U_{\sigma}$. It is equipped with a well-defined blow-down map $\beta:\left[\mathbb{R}_{+}^{n} ; R\right] \longrightarrow \mathbb{R}_{+}^{n}$ given by $\beta_{\sigma}$ on each $U_{\sigma}$.

Proof. The space $U_{\sigma}$, with its local blow-down map $\beta_{\sigma}$, really depends on the choice of the order of the generators implicit in the definition of the map. However, the coordinates $t_{j}$ are each naturally associated to the corresponding generator and change of order of the generators corresponds to the same reordering of the coordinates in $U_{\sigma}$. Thus we can freely reorder the generators.

For two maximal monoids, $\sigma_{1}$ and $\sigma_{2}, \tau=\sigma_{1} \cap \sigma_{2}$ is generated by the common generators of $\sigma_{1}$ and $\sigma_{2}$. For convenience of notation we can assume that these are the first $k$ generators:

$$
\begin{gathered}
\tau=\mathbb{N}\left\langle v_{1}, \ldots, v_{k}\right\rangle=\sigma_{1} \cap \sigma_{2}, \\
\sigma_{1}=\mathbb{N}\left\langle v_{1}, \ldots, v_{k}, v_{k+1}, \ldots, v_{n}\right\rangle, \sigma_{2}=\mathbb{N}\left\langle v_{1}, \ldots, v_{k}, v_{k+1}^{\prime}, \ldots, v_{n}^{\prime}\right\rangle .
\end{gathered}
$$

Since the $\sigma_{i}$ are smooth, their generators form bases so for $i>k$,

$$
v_{i}=\sum_{j=1}^{k} b_{i j} v_{j}+\sum_{j=k+1}^{n} c_{i j} v_{j}^{\prime}, b_{i j}, c_{i j} \in \mathbb{Q},
$$

where the coefficients are rational since both bases are integral with respect to $\left\{x_{1} \partial_{x_{1}}, \ldots, x_{n} \partial_{x_{n}}\right\}$. 
Now, the map $\chi_{12}=\beta_{\sigma_{2}}^{-1} \beta_{\sigma_{1}}$ between the interiors of the two cones is given by $t \longmapsto t^{\mu}=t^{\prime}$, where

$$
\mu=\left(\begin{array}{cc}
\mathrm{Id} & 0 \\
b & c
\end{array}\right) \quad[b]_{i j}=b_{i j},[c]_{i j}=c_{i j}
$$

In other words,

$$
\begin{gathered}
\chi_{12}\left(t_{1}, \ldots, t_{n}\right)=\left(t_{1}^{\prime}, \ldots, t_{n}^{\prime}\right) \in \mathbb{R}_{+}^{n}=U_{\sigma_{2}}, \text { where } \\
t_{i}^{\prime}=t_{i} \prod_{j>k} t_{j}^{b_{j i}}, i \leq k, \quad t_{i}^{\prime}=\prod_{j>k} t_{j}^{c_{j i}}, i>k .
\end{gathered}
$$

Since, in $U_{\sigma_{1}, \tau}, t_{j}>0$ for $j>k, \chi_{12}$ extends smoothly from the interior to $U_{\sigma_{1}, \tau}$. Since the inverse, $\chi_{21}\left(t^{\prime}\right)=\left(t^{\prime}\right)^{\nu^{-1}}$, has a similar form, $\chi_{12}$ is a diffeomorphism onto $U_{\sigma_{2}, \tau}$.

Next we show that the quotient (3.2) is Hausdorff. Consider points $p_{i} \in U_{\sigma_{i}}$, $i=1,2$, which are not identified by $\chi_{12}$. If $p_{1} \in U_{\sigma_{1}, \tau}$, then its image lies in $U_{\sigma_{2}, \tau}$ and a sufficiently small neighborhood of $p_{2}$ does not contain $\chi_{12}\left(p_{1}\right)$ in its closure, so the points have neighborhoods in the $U_{\sigma_{i}}$ with no $\chi_{12}$ related points. Using the inverse the same is true if $p_{2} \in U_{\sigma_{2}, \tau}$, so we may assume both are in the complements of the respective $U_{\sigma_{i}, \tau}$.

Since $\sigma_{1} \cap \sigma_{2}=\tau$, there is a separating hyperplane $u^{\perp}$ for $u \in{ }^{\mathrm{b}} N^{*}\{0\}$ so that

$$
\begin{gathered}
\left\langle u, v_{i}\right\rangle=0, i=1, \ldots, k, \\
\left\langle u, v_{i}\right\rangle>0,\left\langle u, v_{i}^{\prime}\right\rangle<0, i=k+1, \ldots, n .
\end{gathered}
$$

Let $w$ and $w^{\prime}$ be the coordinates of $u$ with respect to the bases dual to the generators of $\sigma_{1}$ and $\sigma_{2}$, respectively. Thus $w_{i}=w_{i}^{\prime}=\left\langle u, v_{i}\right\rangle=0$ for $i=1, \ldots, k$ and $w_{i}=\left\langle u, v_{i}\right\rangle>0, w_{i}^{\prime}=\left\langle u, v_{i}^{\prime}\right\rangle<0$ for $i>k$.

Then $\chi_{12}^{*}\left(t^{\prime}\right)^{w^{\prime}}=t^{w}$, and $p_{1}$ and $p_{2}$ can be separated by the explicit open sets $\left\{t^{w}<\epsilon\right\} \subset U_{\sigma_{1}}$ and $\left\{\left(t^{\prime}\right)^{-w^{\prime}}\right\} \subset U_{\sigma_{2}}$ since

$$
\chi_{12}\left(\left\{t^{w}<\epsilon\right\} \cap U_{\sigma_{1}, \tau}\right)=\left\{\left(t^{\prime}\right)^{w^{\prime}}<\epsilon\right\} \cap U_{\sigma_{2}, \tau}=\left\{\left(t^{\prime}\right)^{-w^{\prime}}>1 / \epsilon\right\} \cap U_{\sigma_{2}, \tau},
$$

so these sets do not meet in the quotient by $\chi_{12}$.

Thus $\left[\mathbb{R}_{+}^{n} ; R\right]$ defined by the quotient (3.2) has the structure of a Hausdorff, paracompact smooth manifold with corners arising from the covering by the coordinate charts which are the images in $\left[\mathbb{R}_{+}^{n}, R\right]$ of the $U_{\sigma}$. Smooth functions on $\left[\mathbb{R}_{+}^{n} ; R\right]$ are those which are smooth in each of the coordinate patches, and the blow-down map

$$
\beta:\left[\mathbb{R}_{+}^{n} ; R\right] \longrightarrow \mathbb{R}_{+}^{n}
$$

is a well-defined smooth b-map since it is such on each coordinate patch; these maps form, by construction, a commutative diagram with the transition maps.

From the point of view of algebraic geometry, the chart $U_{\sigma}$ consists of the ' $\mathbb{R}_{+}$points' of the dual monoid $\widehat{\sigma}$, given by $\operatorname{Hom}_{\text {mon }}\left(\widehat{\sigma}, \mathbb{R}_{+}\right)$(where $\mathbb{R}_{+}$is considered as a multiplicative monoid), though our construction equips this set with a much stronger topology and smooth structure. In this sense $\left[\mathbb{R}_{+}^{n} ; R\right]$ is related to the "singular manifold with corners" Ful93 of a toric variety, though our setup is more closely related to toroidal embeddings KKMSD73 and logarithmic geometry Kat94, Ogu06. It is important to point out however that the gluings between the affine charts $U_{\sigma}$ above are extremely ill-behaved over $\mathbb{R}$ or $\mathbb{C}$ (as opposed to $\mathbb{R}_{+}$here) owing to the presence of radicals (i.e. fractional powers of the coordinates), so what 
results in a smooth manifold with corners above results in a much more singular object (like a stack) in the conventional algebraic setting. This is the ultimate reason for our non-standard definition of monoid refinement in the previous section and for the greater flexibility of our methods.

Next we observe that the boundary faces of $\left[\mathbb{R}_{+}^{n} ; R\right]$ are in bijection with the monoids in the refinement $R$. If $\tau \in R$ and $\sigma \geq \tau$ is a maximal monoid of which it is a face, then $\tau$ defines the boundary face $F_{\sigma, \tau} \subset U_{\sigma}=\mathbb{R}_{+}^{n}$ which is the closure of $U_{\sigma, \tau} \backslash(0, \infty)^{n}$, the part of the boundary where the coordinates corresponding to the generators of $\tau$ vanish (and the other coordinates may or may not vanish).

Proposition 3.2. The manifold $\left[\mathbb{R}_{+}^{n} ; R\right]$, determined by a smooth resolution of $\sigma_{\mathbb{R}_{+}^{n}}$, has interior diffeomorphic to $(0, \infty)^{n}$ and its boundary faces are in 1-1 correspondence with the monoids $\tau \in R$ where the face $F_{\tau}$ corresponding to $\tau$ is the quotient in (3.2) of the union of the $F_{\sigma, \tau} \subset U_{\sigma}$ for the maximal monoids $\sigma$ with $\tau \leq \sigma$. This correspondence satisfies $\operatorname{codim}\left(F_{\tau}\right)=\operatorname{dim}(\tau)$ and is inclusion-reversing:

$$
F_{\tau^{\prime}} \subseteq F_{\tau} \Longleftrightarrow \tau \leq \tau^{\prime}
$$

Proof. The boundary faces of each $U_{\sigma}=\mathbb{R}_{+}^{n}$ are, as follows from (3.1), precisely the $F_{\sigma, \tau}$ for all faces $\tau \leq \sigma$, since these correspond to all subsets of the coordinate functions. Under the transition maps $\chi_{12}$ defined above and corresponding to two monoids, $\sigma_{1}$ and $\sigma_{2}$, of maximal dimension, the interior of each $F_{\sigma_{1}, \tau}$ is mapped onto the interior of $F_{\sigma_{2}, \tau}$ if $\tau \leq \sigma_{1} \cap \sigma_{2}$. Otherwise if $\tau$ is not contained in either $\sigma_{1}$ or $\sigma_{2}$, then $F_{\sigma_{1}, \tau}$ does not meet the domain of definition of $\chi_{12}$ or does not meet the range.

Thus the interiors of the $F_{\sigma, \tau}$ are globally well-defined subsets of $\left[\mathbb{R}_{+}^{n} ; R\right]$. For each $\tau \in R$, the closure in $\left[\mathbb{R}_{+}^{n} ; R\right]$ of the interior of $F_{\sigma, \tau}$ is the image of the union of the closures in the $U_{\sigma}$ that it meets. It is therefore everywhere locally a manifold with corners, and hence is globally a manifold with corners. In particular, each boundary face is embedded in $\left[\mathbb{R}_{+}^{n} ; R\right]$. Finally, the fact that $\tau \leq \tau^{\prime} \Longleftrightarrow F_{\tau^{\prime}} \subseteq F_{\tau}$ is evident in $U_{\sigma}$ for a maximal monoid $\sigma \geq \tau^{\prime} \geq \tau$.

Now we establish a local version of a result we shall prove in more generality in Section [ 6] about lifting b-maps to a generalized blow-up. Suppose

$$
f: O \subset \mathbb{R}_{+}^{m} \longrightarrow \mathbb{R}_{+}^{n}
$$

is an interior b-map with its domain an open set $O \subset \mathbb{R}_{+}^{m}$. Without loss of generality we may assume that $0 \in O$ and that $f(0)=0$. Then $f$ has the explicit coordinate expression

$$
f: x \longmapsto x^{\prime}=a(x) x^{\delta}=\left(a_{1}(x) \prod_{j=1}^{m} x_{j}^{\delta_{j 1}}, \ldots, a_{n}(x) \prod_{j=1}^{m} x_{j}^{\delta_{j n}}\right),
$$

where $\delta \in \operatorname{Mat}(m \times n, \mathbb{N})$ and $0<a_{i}(x) \in C^{\infty}\left(\mathbb{R}_{+}^{m}\right)$. Because of the non-negativity and integrality of the $\delta_{i j}$, the b-differential ${ }^{\mathrm{b}} f_{*}:{ }^{\mathrm{b}} N_{0}\{0\} \longrightarrow{ }^{\mathrm{b}} N_{0}\{0\}$ (which is represented by the matrix $\delta^{\mathrm{T}}$ with respect to the bases $\left\{x_{i} \partial_{x_{i}}\right\}$ and $\left.\left\{x_{i}^{\prime} \partial_{x_{i}^{\prime}}\right\}\right)$ restricts to a monoid homomorphism

$$
{ }^{\mathrm{b}} f_{*}=\delta^{\mathrm{T}}: \sigma_{\mathbb{R}_{+}^{m}} \longrightarrow \sigma_{\mathbb{R}_{+}^{n}} .
$$

(This is a preliminary version of the morphism of monoidal complexes associated to a b-map in Section [6]) 
Proposition 3.3. If $f$ is an interior b-map as above such that ${ }^{\mathrm{b}} f_{*}: \sigma_{\mathbb{R}_{+}^{m}} \longrightarrow \sigma_{\mathbb{R}_{+}^{n}}$ factors through a monoid homomorphism $\phi: \sigma_{\mathbb{R}_{+}^{m}} \longrightarrow \tau$ for some $\tau \in \stackrel{R}{R}$ where $\stackrel{+}{R}$ is a smooth refinement of $\sigma_{\mathbb{R}_{+}^{n}}$, then there exists a unique b-map $f^{\prime}: O \subset \mathbb{R}_{+}^{m} \longrightarrow$ $\left[\mathbb{R}_{+}^{n} ; R\right]$ such that

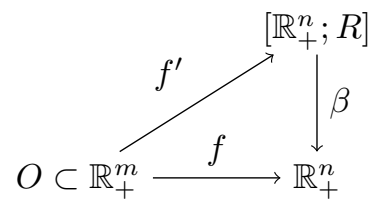

commutes and the range of $f^{\prime}$ is contained in the coordinate chart $U_{\tau} \subset\left[\mathbb{R}_{+}^{n} ; R\right]$. Moreover, this construction is functorial in that for a b-map $g: U \subset \mathbb{R}_{+}^{l} \longrightarrow O \subset$ $\mathbb{R}_{+}^{m},{ }^{\mathrm{b}}(f \circ g)_{*}$ factors through $\tau \in R$ if and only if ${ }^{\mathrm{b}} f_{*}$ does, and then $(f \circ g)^{\prime}=f^{\prime} \circ g$.

Proof. Taking a maximal dimension monoid containing $\tau$ if necessary, it suffices to assume that $\operatorname{dim}(\tau)=n$. Then let $\left(U_{\tau}, t=\left(t_{1}, \ldots, t_{n}\right)\right)$ be the coordinate chart associated to $\tau=\mathbb{N}\left\langle v_{1}, \ldots, v_{n}\right\rangle \in R$, and let $\nu \in \operatorname{GL}(n, \mathbb{N})$ denote the matrix such that $v_{i}=\sum_{j} \nu_{i j} x_{j}^{\prime} \partial_{x_{j}^{\prime}}$ as in the construction of generalized blow-up, so that the blow-down acts by $\beta: t \longmapsto x=t^{\nu}$ on $U_{\tau}$. Alternatively, one can view $\nu^{\mathrm{T}}$ as the matrix defining the monoid inclusion $\nu^{\mathrm{T}}: \tau \hookrightarrow \sigma_{\mathbb{R}_{+}^{n}}$ with respect to the bases $\left\{v_{i}\right\}$ and $\left\{x_{i}^{\prime} \partial_{x_{i}^{\prime}}\right\}$.

In a similar manner, let $\mu \in \operatorname{Mat}(m \times n, \mathbb{N})$ denote the matrix whose transpose represents the given homomorphism

$$
\phi=\mu^{\mathrm{T}}: \sigma_{\mathbb{R}_{+}^{m}} \longrightarrow \tau=\mathbb{N}\left\langle v_{1}, \ldots, v_{n}\right\rangle
$$

with respect to the bases $\left\{x_{i} \partial_{x_{i}}\right\}$ and $\left\{v_{i}\right\}$. From the assumption that ${ }^{\mathrm{b}} f_{*}$ factors through $\phi$ it follows that

$$
\delta^{\mathrm{T}}=\nu^{\mathrm{T}} \mu^{\mathrm{T}}=(\mu \nu)^{\mathrm{T}},
$$

where $\delta$ is the matrix of coefficients of ${ }^{\mathrm{b}} f_{*}$ as above.

Define $f^{\prime}: \mathbb{R}_{+}^{m} \longrightarrow U_{\tau}$ in coordinates by

$$
f^{\prime}: x \longmapsto a(x)^{\nu^{-1}} x^{\mu}=t .
$$

Then observe that $\beta \circ f^{\prime}$ has the form

$$
\beta \circ f^{\prime}: x \longmapsto\left(a(x)^{\nu^{-1}} x^{\mu}\right)^{\nu}=a(x) x^{\mu \nu}=a(x) x^{\delta}
$$

and is therefore equal to $f$. The form of any $f^{\prime}: \mathbb{R}_{+}^{m} \longrightarrow U_{\tau}$ such that $\beta \circ f^{\prime}=f$ is determined in these local coordinates, giving uniqueness.

To show functoriality, suppose that $g: \mathbb{R}_{+}^{l} \longrightarrow \mathbb{R}_{+}^{m}$ acts by $z \longmapsto b(z) z^{\kappa}=x$. We have the commutative diagram

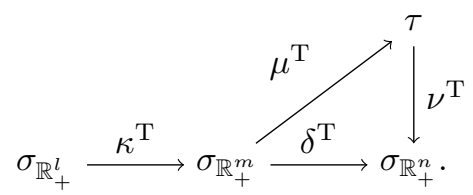

The composition $f \circ g$ acts by

$$
f \circ g: z \longmapsto a\left(b(z) z^{\kappa}\right) b^{\delta} z^{\kappa \delta}=: c(z) z^{\kappa \delta} .
$$


Then on one hand $(f \circ g)^{\prime}$ has the form

$$
(f \circ g)^{\prime}: z \longmapsto c(z)^{\nu^{-1}} z^{\kappa \mu}=a\left(b(z) z^{\kappa}\right)^{\nu^{-1}} b^{\delta \nu^{-1}} z^{\kappa \mu},
$$

while on the other hand $f^{\prime} \circ g$ acts by

$$
f^{\prime} \circ g: z \longmapsto a\left(b(z) z^{\kappa}\right)^{\nu^{-1}}\left(b z^{\kappa}\right)^{\mu},
$$

which is the same since $\delta \nu^{-1}=\mu$.

This result has an important corollary which is fundamental to the global construction of generalized blow-up in Section 6 .

Corollary 3.4. Any diffeomorphism of open submanifolds of $\mathbb{R}_{+}^{n}, f: O_{1} \subset \mathbb{R}_{+}^{n} \cong$ $\mathrm{O}_{2} \subset \mathbb{R}_{+}^{n}$, which maps boundary hypersurfaces onto themselves lifts to a unique diffeomorphism,

$$
f^{\prime}: O_{1}^{\prime} \subset\left[\mathbb{R}_{+}^{n} ; R\right] \longrightarrow O_{2}^{\prime} \subset\left[\mathbb{R}_{+}^{n} ; R\right]
$$

where $O_{i}^{\prime}=\beta^{-1}\left(O_{i}\right)$, such that the diagram

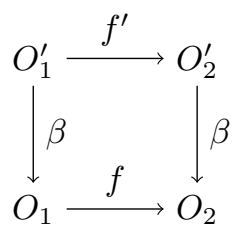

commutes and then $f^{\prime}$ also maps boundary hypersurfaces onto themselves.

Proof. The lift $f^{\prime}$ will be defined locally on coordinate patches, so let $\tau \in R$ be a maximal monoid and consider the b-map

$$
f \circ \beta: O_{1}^{\prime} \cap U_{\tau} \subset \mathbb{R}_{+}^{n} \longrightarrow \mathbb{R}_{+}^{n} .
$$

It is evident that ${ }^{\mathrm{b}}(f \circ \beta)_{*}$ sends $\tau$ (identified here with $\sigma_{\mathbb{R}_{+}^{n}}$ where $\mathbb{R}_{+}^{n}=U_{\tau}$ ) isomorphically to $\tau$, and so it follows from Proposition 3.3 that there is a unique

$$
f_{\tau}^{\prime}=(f \circ \beta)^{\prime}: O_{1}^{\prime} \cap U_{\tau} \longrightarrow U_{\tau}
$$

such that $\beta \circ f_{\tau}^{\prime}=f \circ \beta$. Proceeding this way for each maximal $\tau \in R$, we obtain b-maps (3.3) defined locally on the covering $\left\{O_{1}^{\prime} \cap U_{\tau}\right\}$ of $O_{1}^{\prime}$, and these maps patch together to give a map $f^{\prime}: O_{1}^{\prime} \longrightarrow\left[\mathbb{R}_{+}^{n} ; R\right]$ by uniqueness, with range contained in $\mathrm{O}_{2}^{\prime}$.

Before proving that $f^{\prime}$ is a diffeomorphism, consider the functoriality of these lifts. Thus if $g: O_{2} \subset \mathbb{R}_{+}^{n} \longrightarrow O_{3} \subset \mathbb{R}_{+}^{n}$ is another such diffeomorphism, it follows by the uniqueness and functoriality results in Proposition 3.3 that

$$
(g \circ f)^{\prime}=(g \circ f \circ \beta)^{\prime}=\left(g \circ \beta \circ f^{\prime}\right)^{\prime}=g^{\prime} \circ f^{\prime}: O_{1}^{\prime} \longrightarrow O_{3}^{\prime} .
$$

It is also evident from the proof of Proposition 3.3 that $\mathrm{Id}^{\prime}=\mathrm{Id}$, and it then follows that $f^{\prime}$ is a diffeomorphism with inverse $\left(f^{-1}\right)^{\prime}$.

The following is also a straightforward consequence of Proposition 3.3

Corollary 3.5. If $R(\tau)$ is the refinement of $\tau=\mathbb{N}\left\langle x_{i} \partial_{x_{i}}\right\rangle_{i \in I} \leq \sigma_{\mathbb{R}_{+}^{n}}$ obtained from a smooth refinement $R$ of $\sigma_{\mathbb{R}_{+}^{n}}$, where $I \subset\{1, \ldots, n\}$, and $\operatorname{dim}(\tau)=\# I=k$, there is an injection

$$
\left[\mathbb{R}_{+}^{k} ; R(\tau)\right] \times(0, \infty)^{n-k} \longleftrightarrow\left[\mathbb{R}_{+}^{n} ; R\right]
$$


giving a commutative diagram

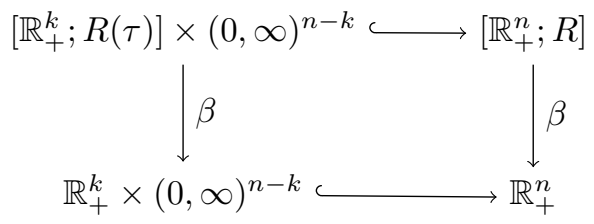

where the bottom map is the inclusion

$$
\mathbb{R}_{+}^{k} \times(0, \infty)^{n-k} \cong\left\{x_{i} \neq 0 ; i \notin I\right\} \subset \mathbb{R}_{+}^{n} .
$$

Proof. The proof is similar to the previous one: the composition of the blow-down and inclusion from $\left[\mathbb{R}_{+}^{k} ; R(\tau)\right] \times(0, \infty)^{n-k}$ to $\mathbb{R}_{+}^{n}$, considered locally on charts in the domain, sends monoids in $R(\tau)$ to the same monoids in $R$, and so factors uniquely (and injectively) through $\left[\mathbb{R}_{+}^{n} ; R\right]$.

Finally we note the following versions of Proposition 3.3 and Corollary 3.4 involving additional factors without boundary. The proofs differ only in notation, where the additional factors are simply carried along.

Proposition 3.6. If $f: O \subset \mathbb{R}_{+}^{m} \times \mathbb{R}^{m^{\prime}} \longrightarrow \mathbb{R}_{+}^{n} \times \mathbb{R}^{n^{\prime}}$ is an interior b-map from an open set containing 0 such that ${ }^{\mathrm{b}} f_{*}: \sigma_{\mathbb{R}_{+}^{m}} \longrightarrow \sigma_{\mathbb{R}_{+}^{n}}$ factors through a monoid homomorphism $\phi: \sigma_{\mathbb{R}_{+}^{m}} \longrightarrow \tau$ for some $\tau \in R$ where $R$ is a smooth refinement of $\sigma_{\mathbb{R}_{+}^{n}}$, then there exists a unique b-map $f^{\prime}$ lifting $f$ such that

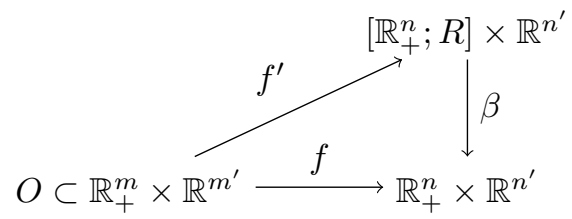

commutes, and these lifts are functorial.

Corollary 3.7. Any diffeomorphism of open submanifolds $f: O_{1} \subset \mathbb{R}_{+}^{n} \times \mathbb{R}^{n^{\prime}} \stackrel{\cong}{\longrightarrow}$ $O_{2} \subset \mathbb{R}_{+}^{n} \times \mathbb{R}^{n^{\prime}}$ which maps boundary hypersurfaces onto themselves lifts to a unique diffeomorphism

$$
f^{\prime}: O_{1}^{\prime} \subset\left[\mathbb{R}_{+}^{n} ; R\right] \times \mathbb{R}^{n^{\prime}} \longrightarrow O_{2}^{\prime} \subset\left[\mathbb{R}_{+}^{n} ; R\right] \times \mathbb{R}^{n^{\prime}},
$$

where $O_{i}^{\prime}=(\beta \times \mathrm{Id})^{-1}\left(O_{i}\right)$, and then $(\beta \times \mathrm{Id}) \circ f^{\prime}=f \circ(\beta \times \mathrm{Id})$.

\section{Monoidal COMPleXes}

In Section 6 we show how to associate a natural monoid to each boundary face of a manifold with corners, with the compatibility condition that whenever $F \subseteq G$, the monoid associated to $G$ is a face of the one associated to $F$, i.e. that face relations persist in an inclusion-reversing sense. We have already seen such an example, which is the association between monoids in $R$ and faces in $\left[\mathbb{R}_{+}^{n} ; R\right]$, where $R$ is a refinement of $\sigma_{\mathbb{R}_{+}^{n}}$. The notion of a refinement (where all the monoids reside in the same vector space) is not sufficiently flexible to capture the ways in which boundary faces may be related for general $X$, so we introduce the concept of a monoidal complex; this should be thought of by analogy to a simplicial or $\mathrm{CW}$ complex. It 
is a generalization of the structure of a refinement in which the monoids are still "attached together nicely along faces", but in which the monoids reside in separate vector spaces and there is no 'base' monoid (as when $R$ is a refinement "of $\sigma$ "). The contents of this section are all that is needed for the discussion of the generalized blow-up of a manifold in Section 6, apart from Theorem 6.5 which uses the fiber product of monoidal complexes discussed in the next section.

Consider a finite partially ordered set (poset) by $A=(A, \leq)$. It is convenient to think of $A$ as a category, with objects the elements $a \in A$ and morphisms given by the order relation: $a \rightarrow b \in \operatorname{Hom}(a, b) \Longleftrightarrow a \leq b$.

Definition 4.1. Let $(A, \leq)$ be a poset. A monoidal complex $\mathcal{Q}$ over $A$ is a covariant functor from $A$ to the category of monoids, where all morphisms are isomorphisms onto faces, so $\mathcal{Q}$ consists of a monoid $\sigma_{a}$ for each $a \in A$, along with isomorphisms called face maps,

$$
i_{a b}: \sigma_{a} \stackrel{\cong}{\longrightarrow} \tau \leq \sigma_{b} \text { whenever } a \leq b,
$$

for some $\tau \leq \sigma_{b}$. This relation will be denoted

$$
\sigma_{a} \leq \sigma_{b}, \text { for } a \leq b .
$$

We say $\mathcal{Q}$ is complete, respectively reduced, if for every $b \in A$ and every face $\tau \leq \sigma_{b}$ there exists at least one (resp. at most one) $a \in A$ such that $a \leq b$ and $i_{a b}: \sigma_{a} \cong \tau$.

Lemma 4.2. If $\mathcal{Q}$ is complete and reduced, then the posets $(A, \leq)$ and $\Gamma_{\mathcal{Q}}=$ $\{\tau \leq \sigma ; \sigma \in \mathcal{Q}\} / i_{*}$ with the order (4.2) are isomorphic.

Proof. Consider the composition of the map $A \ni a \longmapsto \sigma_{a} \in \mathcal{Q}$ with the quotient by the face maps (4.1). This map $A \longrightarrow \Gamma_{\mathcal{Q}}$ intertwines the orders by (4.2); it must be injective by the assumption that $\mathcal{Q}$ is reduced and be surjective by the assumption that it is complete.

From this it follows that for a complete, reduced monoidal complex, $A$ is entirely determined by the set of $\sigma \in \mathcal{Q}$ and the face relations (4.2). Nevertheless, it is often convenient to be able to refer explicitly to an indexing set $A$, as in the case $(A, \leq)=(\mathcal{M}(X), \leq)$ below, which we will most often encounter.

There are evidently some obstructions for a general poset to index a complete, reduced monoidal complex, but we shall not concern ourselves with them here; all complete reduced monoidal complexes will arise naturally.

From now on, a monoidal complex will mean a complete and reduced monoidal complex.

Example 4.3. The faces of a single monoid $\sigma$ form a monoidal complex over the poset $\{\tau ; \tau \leq \sigma\}$.

More generally, any refinement $R$ of $\sigma$ forms a monoidal complex over the set $R$ with the order coming from the face relations.

For an example which is not a refinement, consider the following.

Example 4.4. Let $\sigma_{0}=\{0\}, \sigma_{a}=\mathbb{N}(1,0) \subset \mathbb{R}^{2}, \sigma_{b}=\mathbb{N}(0,1) \subset \mathbb{R}^{2}$, and let $\sigma_{c}$ and $\sigma_{d}$ be two distinct copies of the monoid $\mathbb{N}\langle(1,0),(0,1)\rangle \subset \mathbb{R}^{2}$. Let the face maps 


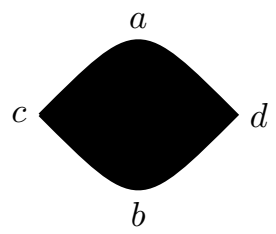

Figure 2. A manifold whose boundary face relations do not index a monoid refinement.

be the obvious ones in the diagram

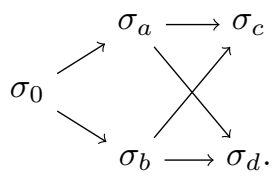

This complex cannot be realized as a refinement, since otherwise $\sigma_{c}$ and $\sigma_{d}$ would have to be identified; here they remain distinct. The poset underlying this complex is the same as the one given by the boundary faces (with the order of reverse inclusion) of the 2-dimensional manifold with corners pictured in Figure 2 .

Definition 4.5. A morphism of monoidal complexes $\phi: \mathcal{Q}_{A} \longrightarrow \mathcal{Q}_{B}$ consists of a map of posets $\phi_{\#}:(A, \leq) \longrightarrow(B, \leq)$ and monoid homomorphisms $\phi_{a b}: \mathcal{Q}_{A} \ni$ $\sigma_{a} \longrightarrow \sigma_{b} \in \mathcal{Q}_{B}$ for all $a \in A$, where $b=\phi_{\#}(a) \in B$. These are required to commute with the homomorphisms $i_{a a^{\prime}}$ for $a \leq a^{\prime}$, so that

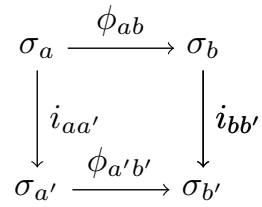

commutes, where $a \leq a^{\prime}, \phi_{\#}(a)=b, \phi_{\#}\left(a^{\prime}\right)=b^{\prime}$, and thus $b \leq b^{\prime}$. We say $\phi$ is injective if all the morphisms $\phi_{a b}$ are injective, though we do not necessarily require that $\phi_{\#}: A \longrightarrow B$ be injective.

An elementary example of a morphism is the inclusion of a subcomplex:

Definition 4.6. Let $\mathcal{Q}$ be a monoidal complex over $(A, \leq)$. A monoidal subcomplex of $\mathcal{Q}$ is a complex $\mathcal{Q}_{0}$ obtained by restricting $\mathcal{Q}$ to a subset $A_{0} \subset A$ :

$$
\mathcal{Q}_{0}=\left\{\sigma_{a} ; a \in A_{0}\right\}
$$

such that $\mathcal{Q}_{0}$ is complete and reduced. There is then an injective morphism

$$
\mathcal{Q}_{0} \longleftrightarrow \mathcal{Q}
$$

consisting of the identity homomorphisms over $A_{0} \subset A$.

In Section 6, we will show that a b-map $f: X \longrightarrow Y$ gives rise to a morphism $f_{\natural}: \mathcal{P}_{X} \longrightarrow \mathcal{P}_{Y}$ of the basic monoidal complexes associated to $X$ and $Y$.

Another important class of morphisms consists of refinements. 


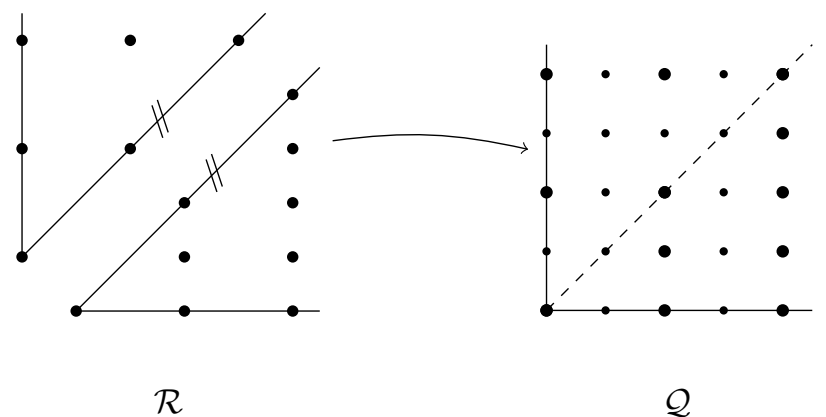

Figure 3. A refinement of monoidal complexes. Only maximal dimension monoids are pictured. In this example, $\mathcal{R} \rightarrow \mathcal{Q}$ is injective, though $\mathcal{R}$ is not smooth, and the individual monoid homomorphisms do not cover their targets.

Definition 4.7. Let $\mathcal{Q}$ be a monoidal complex. A refinement of $\mathcal{Q}$ is a morphism $\phi: \mathcal{R} \longrightarrow \mathcal{Q}$ all of whose homomorphisms are injective, and for all $\sigma \in \mathcal{Q}$,

(i) $\bigcup_{\tau \in \phi_{\#}^{-1}(\sigma)} \phi(\operatorname{supp}(\tau))=\operatorname{supp}(\sigma)$ and

(ii) for $\tau_{1}, \tau_{2} \in \phi_{\#}^{-1}(\sigma), \operatorname{relint}\left[\phi\left(\operatorname{supp}\left(\tau_{1}\right)\right)\right] \cap \operatorname{relint}\left[\phi\left(\operatorname{supp}\left(\tau_{2}\right)\right)\right]=\emptyset$ unless $\tau_{1}=$ $\tau_{2}$,

where the relative interior of a cone $C=\operatorname{span}_{\mathbb{R}_{+}}\left\{v_{1}, \ldots, v_{k}\right\}$ is the set relint $[C]=$ $\operatorname{span}_{(0, \infty)}\left\{v_{1}, \ldots, v_{k}\right\}$.

Condition (ii) requires that the support of each monoid in $\mathcal{Q}$ is covered by the supports of monoids in $\mathcal{R}$, and condition (iii) demands that these supports only intersect along mutual faces. These, along with our assumption that $\mathcal{R}$ is complete and reduced, are analogous to the conditions in Definition 2.2 for the refinement of a monoid. Figure 3 depicts a refinement.

Proposition 4.8. If $\phi: \mathcal{R} \longrightarrow \mathcal{Q}$ is a refinement, then for each $\sigma \in \mathcal{Q}$, the collection

$$
\mathcal{R}(\sigma):=\left\{\phi\left(\sigma^{\prime}\right) ; \sigma^{\prime} \in \phi_{\#}^{-1}(\tau), \tau \leq \sigma\right\}
$$

is a refinement of $\sigma$. Conversely, let $\{R(\sigma) ; \sigma \in \mathcal{Q}\}$ be a collection of refinements which are compatible in that whenever $\tau \leq \sigma$, the refinement $R(\tau)$ is identical to the induced refinement $(R(\sigma))(\tau)$ as in Lemma 2.4. Then the quotient $\mathcal{R}=\bigcup R(\sigma) / i_{*}$ of the set of all monoids in the collection by the face maps $i_{*}$ of $\mathcal{Q}$ forms a refinement $\phi: \mathcal{R} \longrightarrow \mathcal{Q}$ of monoidal complexes.

Proof. Let $\mathcal{R}(\sigma)$ be the set in (4.3). Certainly

$$
\mathcal{R}(\sigma) \supseteq\left\{\phi\left(\sigma^{\prime}\right) ; \sigma^{\prime} \in \phi_{\#}^{-1}(\sigma)\right\},
$$

and it follows that $\mathcal{R}(\sigma)$ satisfies property (iii) of Definition 2.2 for a refinement of $\sigma$ by property (ii) in Definition 4.7 above.

To see that $\mathcal{R}(\sigma)$ satisfies the other two properties of a refinement, let $\tau_{i}^{\prime} \in \mathcal{R}$, $i=1,2$, with $\phi\left(\tau_{i}^{\prime}\right) \in \mathcal{R}(\sigma)$, and for convenience of notation, identify these with their images in $\sigma$. Let $\tau \leq \sigma$ be the smallest face such that $\tau_{1}^{\prime} \cap \tau_{2}^{\prime} \subset \tau$. Since $\phi$ is 
a morphism it follows that each $\tau_{i}^{\prime} \cap \tau$ is a face of $\tau_{i}^{\prime}$, hence also in $\mathcal{R}$, and it must be that $\tau_{i}^{\prime} \cap \tau \in \phi_{\#}^{-1}(\tau)$ (or else there would be a smaller $\tau$ ), so it follows that the face $\tau_{i}^{\prime} \cap \tau \leq \tau$ is also in $\mathcal{R}(\sigma)$.

By property (iii) above for $\tau_{i}^{\prime} \cap \tau \in \phi_{\#}^{-1}(\tau)$, it follows that $\tau_{1}^{\prime} \cap \tau_{2}^{\prime}=\left(\tau_{1}^{\prime} \cap \tau\right) \cap$ $\left(\tau_{2}^{\prime} \cap \tau\right)$ must be a face of each. To see that $\mathcal{R}(\sigma)$ contains the faces of all its monoids, simply let $\tau_{1}^{\prime}$ be the face in question of $\tau_{2}^{\prime}$; we conclude that $\tau_{1}^{\prime}=\tau_{1}^{\prime} \cap \tau_{2}^{\prime} \in$ $\mathcal{R}(\sigma)$. Thus $\mathcal{R}(\sigma)$ is a refinement of $\sigma$.

For the converse, let $\mathcal{R}$ be the set of all monoids in the refinements $R(\sigma), \sigma \in \mathcal{Q}$, modulo the identification of monoids in $(R(\sigma))(\tau)$ with those in $R(\tau)$ for $\tau \leq \sigma$, as above. That $\mathcal{R}$ is a complex follows easily from the fact that each $R(\sigma)$ forms a monoidal complex.

For notational clarity, denote the posets for $\mathcal{R}$ and $\mathcal{Q}$ by $(A, \leq)$ and $(B, \leq)$, respectively. We take $\phi_{\#}: A \longrightarrow B$ to be the map which sends $a$ to the smallest $b \in B$ such that $\sigma_{a}^{\prime} \in \mathcal{R}$ is in the refinement $R_{\sigma_{b}}$, and then $\phi_{a b}: \sigma_{a}^{\prime} \longrightarrow \sigma_{b}$ is given by the inclusion $R_{\sigma_{b}} \ni \sigma_{a}^{\prime} \subset \sigma_{b}$ which is of course injective.

Finally, it follows directly from the fact that the $R(\sigma)$ are refinements that $\phi: \mathcal{R} \longrightarrow \mathcal{Q}$ satisfies the properties in Definition 4.7

The remainder of this section is devoted to specific algorithms for obtaining and extending various refinements of complexes.

A primary means of obtaining refinements is star subdivision; we extend this to a monoidal complex. The proof of the following follows directly from Propositions 4.8 and 2.5

Proposition 4.9 (Star subdivision). Let $\mathcal{Q}$ be a monoidal complex, $\sigma_{a} \in \mathcal{Q}$, and $v \in \sigma_{a}$. The monoids

$$
\mathcal{S}(\mathcal{Q}, v)\left(\sigma_{b}\right)= \begin{cases}S\left(\sigma_{b},\left(i_{a b} v\right)\right) & \text { if } \sigma_{a} \leq \sigma_{b} \text { and } \\ \sigma_{b} & \text { otherwise }\end{cases}
$$

form a monoidal complex which refines $\mathcal{Q}$. If $\mathcal{Q}$ is smooth, then $\mathcal{S}(\mathcal{Q}, v)$ is smooth.

Recall that in Section 2 an extension of star subdivision is discussed, giving a refinement $S(\sigma, \mu)$ of a smooth monoid $\sigma$ with respect to a monoid $\mu$ given by the intersection of $\sigma$ with an integral subspace. We now extend this to monoidal complexes, which will be of use in Section 10.

Proposition 4.10 (Planar refinement). Let $\mathcal{P}$ a smooth monoidal complex and suppose $i: \mathcal{Q} \longrightarrow \mathcal{P}$ is an injective morphism such that for all $\sigma \in \mathcal{P},\left(i_{\#}\right)^{-1}(\sigma) \subset$ $\mathcal{Q}$ contains at most one monoid $\mu$, and if $i(\mu) \subset \sigma$ is the intersection of $\sigma$ with an integral subspace, then

$$
\mathcal{S}(\mathcal{P}, \mathcal{Q})(\sigma)=S(\sigma, \mu), \quad \mu=\left(i_{\#}\right)^{-1}(\sigma),
$$

form a complex, containing $\mathcal{Q}$ as a subcomplex, which refines $\mathcal{P}$.

Proof. This follows directly from Proposition 2.7, using the fact that $S(\tau, \mu \cap \tau)=$ $S(\sigma, \mu)(\tau)$.

The smoothing of a simplicial monoid also extends to monoidal complexes.

Proposition 4.11 (Smoothing). Let $\mathcal{Q}$ be a simplicial complex. Then the collection of monoid refinements $\sigma_{\mathrm{sm}} \longrightarrow \sigma$, where $\sigma_{\mathrm{sm}}=\mathbb{N}\left\langle v_{1}, \ldots, v_{n}\right\rangle$ is freely generated by the extremals of $\sigma$ as in Example 2.3 , forms a smooth refinement $\mathcal{Q}_{\mathrm{sm}} \longrightarrow \mathcal{Q}$, in which the indexing posets are the same. 
Proof. This follows directly from the fact that the faces of $\sigma_{\mathrm{sm}}$, namely the monoids $\mathbb{N}\left\langle v_{i}\right\rangle_{i \in I}, I \subset\{1, \ldots, n\}$, are the smoothings of the corresponding faces of $\sigma$.

\section{Fiber PRODUCTS OF COMPLEXES}

Below we frequently need to address the problem of finding a refinement $\mathcal{R}_{1} \longrightarrow$ $\mathcal{Q}_{1}$ of a complex which is compatible with a given refinement $\mathcal{R}_{2} \longrightarrow \mathcal{Q}_{2}$ with respect to a morphism $\mathcal{Q}_{1} \longrightarrow \mathcal{Q}_{2}$, meaning that

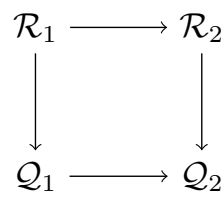

commutes. This section is therefore devoted to the basic constructions and properties of fiber products in the category of monoidal complexes. Apart from the proof of Theorem 6.5, the material here is not used until Section 10 .

First consider fiber products in the categories of posets and monoids. Thus, let $(A, \leq),(B, \leq)$ and $(C, \leq)$ be posets, with order preserving maps $\left(\phi_{1}\right)_{\#}: A \longrightarrow C$ and $\left(\phi_{2}\right)_{\#}: B \longrightarrow C$.

The product $A \times B$ is a poset with elements $(a, b): a \in A, b \in B$ and order given by

$$
\left(a_{1}, b_{1}\right) \leq\left(a_{2}, b_{2}\right) \Longleftrightarrow a_{1} \leq a_{2} \text { and } b_{1} \leq b_{2},
$$

and the fiber product is the subset

$$
A \times_{C} B=\left\{(a, b) \in A \times B ;\left(\phi_{1}\right)_{\#}(a)=\left(\phi_{2}\right)_{\#}(b)=c\right\} \subset A \times B,
$$

with the order induced from $A \times B$, which is well defined since the $\left(\phi_{i}\right)_{\#}$ are order preserving.

Proposition 5.1 (Fiber products of monoids). If $\phi_{i}: \sigma_{i} \longrightarrow \sigma, i=1,2$, are (toric) monoid homomorphisms, then there is a unique toric monoid fiber product $\sigma_{1} \times_{\sigma} \sigma_{2}$, with homomorphisms to $\sigma_{i}$ such that

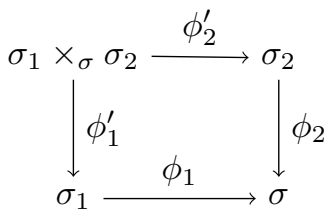

commutes, and which has the usual universal property.

Its faces can be described as follows. Let $A_{1}, A_{2}$ and $A$ be the posets whose elements are faces of $\sigma_{1}, \sigma_{2}$, and $\sigma$, respectively, and define $\left(\phi_{i}\right)_{\#}: A_{i} \longrightarrow A$ by taking $\left(\phi_{i}\right)_{\#}\left(\tau_{i}\right)$ to be the smallest $\tau \in A$ such that $\phi_{i}\left(\tau_{i}\right) \subset \tau$. Then faces of $\sigma_{1} \times_{\sigma} \sigma_{2}$ are indexed by an ordered subset of $A_{1} \times_{A} A_{2}$.

Faces of $\sigma_{1} \times{ }_{\sigma} \sigma_{2}$ are not necessarily in bijection with $A_{1} \times_{A} A_{2}$, since there will generally be distinct pairs $\left(\tau_{1}, \tau_{2}\right) \neq\left(\tau_{1}^{\prime}, \tau_{2}^{\prime}\right) \in A_{1} \times_{A} A_{2}$ (with $\tau_{i}^{\prime} \leq \tau_{i}$ and $\left.\phi_{\#}\left(\tau_{i}\right)=\phi_{\#}\left(\tau_{i}^{\prime}\right)=\tau, i=1,2\right)$ such that $\tau_{1} \times_{\tau} \tau_{2}=\tau_{1}^{\prime} \times_{\tau} \tau_{2}^{\prime}$. 
Proof. $\sigma_{1} \times \sigma_{2}$ is a toric monoid in $N_{\sigma_{1}} \times N_{\sigma_{2}}$, generated by $\left\{\left(v_{i}, 0\right),\left(0, w_{j}\right)\right\}$ where $\left\{v_{1}, \ldots, v_{n}\right\}$ generate $\sigma_{1}$ and $\left\{w_{1}, \ldots, w_{k}\right\}$ generate $\sigma_{2}$, and the set

$$
\sigma_{1} \times \sigma_{\sigma} \sigma_{2}=\left\{(v, w) \in \sigma_{1} \times \sigma_{2} ; \phi_{1}(v)=\phi_{2}(w)\right\}
$$

(which might be trivial) is evidently closed under addition. It is the intersection of $N_{\sigma_{1}} \times N_{\sigma_{2}}$ with the cone

$$
\operatorname{supp}\left(\sigma_{1}\right) \times_{\operatorname{supp}(\sigma)} \operatorname{supp}\left(\sigma_{2}\right)=\operatorname{supp}\left(\sigma_{1}\right) \times \operatorname{supp}\left(\sigma_{2}\right) \cap N_{\sigma_{1}}^{\mathbb{R}} \times_{N_{\sigma}^{\mathbb{R}}} N_{\sigma_{2}}^{\mathbb{R}},
$$

and hence is a toric monoid.

We will construct a map $I$ from faces of $\sigma_{1} \times_{\sigma} \sigma_{2}$ to $A_{1} \times_{A} A_{2}$. Let $\tau \leq \sigma_{1} \times_{\sigma} \sigma_{2}$ be a face. Since $\sigma_{1} \times{ }_{\sigma} \sigma_{2} \subset \sigma_{1} \times \sigma_{2}$, there is a smallest face of $\sigma_{1} \times \sigma_{2}$ containing $\tau$ which must be of the form $\tau_{1} \times \tau_{2}$ for some $\tau_{i} \leq \sigma_{i}, i=1,2$. Observe that $\left(\phi_{1}\right)_{\#}\left(\tau_{1}\right)=\left(\phi_{2}\right)_{\#}\left(\tau_{2}\right) \in A$ : indeed, $\left(\phi_{1}\right)_{\#}\left(\tau_{1}\right)$ and $\left(\phi_{2}\right)_{\#}\left(\tau_{2}\right)$ must have a common face (containing the image of $\tau$ ), and then passing to the inverse images of this face with respect to the $\left(\phi_{i}\right)_{\#}$ would give a smaller face of $\sigma_{1} \times \sigma_{2}$ containing $\tau$ unless $\left(\phi_{1}\right)_{\#}\left(\tau_{1}\right)=\left(\phi_{2}\right)_{\#}\left(\tau_{2}\right)$. Thus $\left(\tau_{1}, \tau_{2}\right) \in A_{1} \times_{A} A_{2}$ and we set $I(\tau)=\left(\tau_{1}, \tau_{2}\right)$.

Next observe that $\tau \leq \tau_{1} \times{ }_{\sigma} \tau_{2}$; however, $\tau$ meets the interior of $\tau_{1} \times \tau_{2}$ (otherwise $\tau_{1} \times \tau_{2}$ would not be minimal) and any proper face of $\tau_{1} \times_{\sigma} \tau_{2}$ would have to lie in a proper face of $\tau_{1} \times \tau_{2}$ (which follows from the fact that $\tau_{1} \times_{\sigma} \tau_{2}$ is the intersection of the lattice $N_{\tau_{1}} \times N_{\tau_{2}}$, the proper convex cone $C=\operatorname{supp}_{\tau_{1}} \times \operatorname{supp}_{\tau_{2}}$ and the subspace $N_{\tau_{1}}^{\mathbb{R}} \times_{N_{\sigma}^{\mathbb{R}}} N_{\tau_{2}}^{\mathbb{R}}$ ), so in fact $\tau=\tau_{1} \times_{\sigma} \tau_{2}$. It follows that $I$ is injective.

The universal property of $\sigma_{1} \times_{\sigma} \sigma_{2}$ in the category of monoids follows from the analogous property in the category of sets, since the maps factoring through $\sigma_{1} \times{ }_{\sigma} \sigma_{2}$ are additive.

Note that $\sigma_{1} \times_{\sigma} \sigma_{2}$ need not be smooth (or even simplicial) even if $\sigma_{1}$ and $\sigma_{2}$ are, as the example $\phi_{1}=\phi_{2}: \mathbb{N}^{2} \longrightarrow \mathbb{N},(m, n) \longmapsto m+n$ shows.

Proposition 5.2 (Fiber product of monoidal complexes). Products and fiber products exist in the category of monoidal complexes. Thus if $\mathcal{Q}_{1}, \mathcal{Q}_{2}$ are monoidal complexes over $A$ and $B$, respectively, and if $\phi_{i}: \mathcal{Q}_{i} \longrightarrow \mathcal{Q}$ are morphisms to a complex $\mathcal{Q}$ over $C$, then there exist (complete and reduced) monoidal complexes $\mathcal{Q}_{1} \times \mathcal{Q}_{2}$ over $A \times B$ and $\mathcal{Q}_{1} \times{ }_{\mathcal{Q}} \mathcal{Q}_{2}$ over $A \times{ }_{C} B$ with the requisite universal properties.

Proof. To define $\mathcal{Q}_{1} \times \mathcal{Q}_{2}$, let $\sigma_{(a, b)}=\sigma_{a} \times \sigma_{b}$ for each $(a, b) \in A \times B$. Likewise, define $\mathcal{Q}_{1} \times{ }_{\mathcal{Q}} \mathcal{Q}_{2}$ to consist of the distinct $\sigma_{(a, b)}=\sigma_{a} \times_{\sigma_{c}} \sigma_{b}$ for $(a, b) \in A \times{ }_{C} B$. The only issue is to show that these are complete and reduced.

Consider first $\mathcal{Q}_{1} \times \mathcal{Q}_{2}$. The faces of $\sigma_{a} \times \sigma_{b}$ are the monoids $\sigma_{a^{\prime}} \times \sigma_{b^{\prime}}$ where $\sigma_{a^{\prime}} \leq \sigma_{a}$ and $\sigma_{b^{\prime}} \leq \sigma_{b}$. Since the $\mathcal{Q}_{i}$ are complete and reduced, these are in bijection with the elements $\left(a^{\prime}, b^{\prime}\right) \in A \times B$ such that $\left(a^{\prime}, b^{\prime}\right) \leq(a, b)$.

Next, it follows from Proposition 5.1 that the faces of $\sigma_{a} \times_{\sigma_{c}} \sigma_{b}$ are monoids of the form $\sigma_{a^{\prime}} \times_{\sigma_{c}} \sigma_{b^{\prime}}=\sigma_{a^{\prime}} \times_{\sigma_{c^{\prime}}} \sigma_{b^{\prime}}$ where $\left(\phi_{1}\right)_{\#}\left(a^{\prime}\right)=\left(\phi_{2}\right)_{\#}\left(b^{\prime}\right)=c^{\prime} \leq c$, and for such a face $\left(a^{\prime}, b^{\prime}\right) \leq(a, b) \in A \times_{C} B$ is unique, so $\mathcal{Q}_{1} \times_{\mathcal{Q}} \mathcal{Q}_{2}$ is complete and reduced.

The universal properties of $\mathcal{Q}_{1} \times \mathcal{Q}_{2}$ and $\mathcal{Q}_{1} \times{ }_{\mathcal{Q}} \mathcal{Q}_{2}$ follow from the corresponding universal properties of $A \times B$ and $A \times{ }_{C} B$ among posets, and of $\sigma_{1} \times \sigma_{2}$ and $\sigma_{1} \times{ }_{\sigma} \sigma_{2}$ among monoids. 
Proposition 5.3 (Refinements pull-back). If $\phi: \mathcal{R} \longrightarrow \mathcal{Q}$ is a refinement and $\psi: \mathcal{Q}_{1} \longrightarrow \mathcal{Q}$ is any morphism of complexes, then

$$
\phi^{\prime}: \mathcal{Q}_{1} \times{ }_{\mathcal{Q}} \mathcal{R} \longrightarrow \mathcal{Q}_{1} \text { is a refinement. }
$$

In particular, the fiber product $\mathcal{R}_{1} \times{ }_{\mathcal{Q}} \mathcal{R}_{2}$ of two refinements is a mutual refinement of each.

Proof. Fix $\sigma \in \mathcal{Q}$, and consider $\tau \in \mathcal{R}(\sigma)$ and $\sigma_{1} \in \mathcal{Q}_{1}$ such that $\psi: \sigma_{1} \longrightarrow \sigma$. If we identify $\tau$ with its image in $\sigma$, then as noted above,

$$
\phi^{\prime}: \sigma_{1} \times_{\sigma} \tau \cong \psi^{-1}(\tau) \longleftrightarrow \sigma_{1}
$$

is identified with an inclusion and therefore injective. From the identity

$$
\psi^{-1}\left(\tau_{1} \cap \tau_{2}\right)=\psi^{-1}\left(\tau_{1}\right) \cap \psi^{-1}\left(\tau_{2}\right)
$$

it then follows that $\sigma_{1} \times_{\sigma} \mathcal{R}(\sigma)=\left\{\sigma_{1} \times_{\sigma} \tau ; \tau \in \mathcal{R}(\sigma)\right\}$ is a refinement of $\sigma_{1}$ and that by commutativity of $\phi$ and $\psi$ with the face maps $\mathcal{Q}_{1} \times{ }_{\mathcal{Q}} \mathcal{R} \longrightarrow \mathcal{Q}_{1}$ forms a refinement of complexes.

The complex $\mathcal{Q}_{1} \times{ }_{\mathcal{Q}} \mathcal{Q}_{2}$ need not be smooth, even if $\mathcal{Q}_{1}$ and $\mathcal{Q}_{2}$ are smooth and even if they are refinements. For this and other reasons, it is desirable to know that smooth refinements exist.

Like the classical algorithms for obtaining smooth refinements in toric geometry (Ful93], [DCP83]), the following algorithm consists of two steps. The first step results in a simplicial refinement and is similar in spirit to the usual algorithms except for the novelty of subdividing monoids from largest dimension downwards rather than the other way - this strategy has the desirable feature of being functorial with respect to the inclusion of complexes. The second step is to produce a smooth refinement from a simplicial one, and here we make use of the smoothing operation of Proposition 4.11 which, as previously noted, is not available in the conventional algebraeo-geometric settings.

Theorem 5.4 (Natural smooth refinement). Let $\mathcal{Q}$ be a monoidal complex. Then there exists a natural smooth refinement $\mathrm{NS}(\mathcal{Q}) \longrightarrow \mathcal{Q}$ with the following properties:

(i) If $\mathcal{Q}$ is smooth, then $\operatorname{NS}(\mathcal{Q})=\mathcal{Q}$.

(ii) If $\mathcal{Q}_{0} \subset \mathcal{Q}$ is a subcomplex, then the corresponding subcomplex of $\mathrm{NS}(\mathcal{Q})$ is the natural smooth refinement of $\mathcal{Q}_{0}$, i.e.

$$
\operatorname{NS}\left(\mathcal{Q}_{0}\right)=\operatorname{NS}(\mathcal{Q})_{0}:=\left\{\operatorname{NS}(\mathcal{Q})(\sigma) ; \sigma \in \mathcal{Q}_{0}\right\}
$$

Proof. Consider a monoid $\sigma$ with extremals $V=\left\{v_{1}, \ldots, v_{n}\right\}$. Let $\Lambda$ be the set of those $v_{i}$ which are linearly independent from all of the others, i.e. such that $\operatorname{span}\left\{v_{i}\right\} \cap \operatorname{span}\left(V \backslash v_{i}\right)=\{0\}$. Then the monoid $\tau$ which is the largest face of $\sigma$ lying in the span of $V \backslash \Lambda$ is uniquely determined and thus is either $\{0\}$ or nonsimplicial. It represents the 'essential' non-simpliciality of $\sigma$, since $\sigma$ consists of the join of $\tau$ and the smooth face generated by $\Lambda$.

We define the non-simplicial dimension of $\sigma$ by

$$
\operatorname{nsdim}(\sigma)=\operatorname{dim}(\tau)
$$

so in particular, $\sigma$ is simplicial if and only if $\operatorname{nsdim}(\sigma)=0$. If $\operatorname{nsdim}(\sigma)=\operatorname{dim}(\sigma)$ (i.e. $\Lambda=\emptyset$, so $\sigma=\tau$ ), we call $\sigma$ fully non-simplicial.

For $j \in \mathbb{N}$, let

$$
M_{j}(\mathcal{Q})=\#\{\sigma ; \operatorname{nsdim}(\sigma)=j\}
$$


Since $\mathcal{Q}$ has a finite number of monoids which are each finite dimensional, each $M_{j}(\mathcal{Q})$ is finite, and $M_{j}(\mathcal{Q})=0$ for all $j \geq N$, for some $N$.

Proceeding by induction, assume that $M_{j}(\mathcal{Q})=0$ for $j>k$. If there are no fully non-simplicial monoids of dimension $k$, then $M_{k}(\mathcal{Q})=0$ (indeed, if $\operatorname{nsdim}(\sigma)=k$, then it has a fully non-simplicial face $\tau \leq \sigma$ as above of dimension $k$ ), and the induction is complete. Otherwise, let $\sigma$ with extremals $\left\{v_{1}, \ldots, v_{n}\right\}$ be a fully nonsimplicial monoid of dimension $k$, and consider the star subdivision $\mathcal{S}(\mathcal{Q}, v)$, where $v=v_{1}+\cdots+v_{n}$.

If $v$ lies in a monoid $\mu \in \mathcal{Q}$, then necessarily $\sigma \leq \mu$, and by the induction hypothesis $k \geq \operatorname{nsdim}(\mu) \geq \operatorname{nsdim}(\sigma)=k$, so equality holds. Moreover, $\sigma$ must be the unique fully non-simplicial face of $\mu$ with dimension $k$, so any face $\tau \leq \mu$ with $\operatorname{nsdim}(\tau)=k$ must have $\sigma \leq \tau$. Thus if $v \notin \tau \leq \mu$, then $\sigma \not \leq \tau$, and therefore $\operatorname{nsdim}(\tau+\mathbb{N} v)<k$ since $v$ is independent from the generators of $\tau$ and $\operatorname{nsdim}(\tau)<k$.

Since all monoids in $\mathcal{S}(\mathcal{Q}, v)$ which are not in $\mathcal{Q}$ are of the form $\tau+\mathbb{N} v$ where $v \notin \tau$ and $\tau \leq \mu \ni v$, it follows that $M_{j}(\mathcal{S}(\mathcal{Q}, v)) \leq M_{j}(\mathcal{Q})$ for $j \geq k$. On the other hand, $M_{k}$ must actually decrease by at least one, since $\mathcal{Q}$ contains a monoid of non-simplicial dimension $k$ which is not in $\mathcal{S}(\mathcal{Q}, v)$, namely $\sigma$. Thus

$$
M_{k}(\mathcal{S}(\mathcal{Q}, v))<M_{k}(\mathcal{Q}),
$$

and since $M_{k}(\mathcal{Q})<\infty$, after a finite number of such subdivisions, we obtain a complex $\mathcal{Q}^{\prime}$ for which $M_{j}\left(\mathcal{Q}^{\prime}\right)=0$ for $j \geq k$ and $M_{j}\left(\mathcal{Q}^{\prime}\right)<\infty$ for $j<k$, completing the inductive step.

Note that the inductive step does not depend on the order of the subdivisions. Indeed, the result of subdividing $\sigma_{1}$ and $\sigma_{2}$ of dimension $k$ in either order is the same, unless there is a monoid $\mu$ with $\sigma_{1} \leq \mu$ and $\sigma_{2} \leq \mu$. But such a $\mu$ would then have $\operatorname{nsdim}(\mu)>k$, contradicting the induction hypothesis. Upon completion of the induction, we obtain a complex $\operatorname{NS}^{\prime}(\mathcal{Q})$ such that $M_{j}\left(\mathrm{NS}^{\prime}(\mathcal{Q})\right)$ vanishes for all $j$; thus $\operatorname{NS}^{\prime}(\mathcal{Q})$ is simplicial. Since star subdivisions are refinements and refinements compose, $\operatorname{NS}^{\prime}(\mathcal{Q}) \longrightarrow \mathcal{Q}$ is a simplicial refinement.

Finally set $\operatorname{NS}(\mathcal{Q})=\operatorname{NS}^{\prime}(\mathcal{Q})_{\mathrm{sm}}$. This involves a local operation on each monoid and does not depend on any choice of order of the monoids. The first property of $\operatorname{NS}(\mathcal{Q})$ is clear, since if $\mathcal{Q}$ is smooth, then $M_{j}(\mathcal{Q})=0$ for all $j$, so $\operatorname{NS}^{\prime}(\mathcal{Q})=\mathcal{Q}$ and $\mathcal{Q}_{\mathrm{sm}}=\mathcal{Q}$.

The second property follows from the fact that either $(\mathcal{S}(\mathcal{Q}, v))_{0}=\mathcal{Q}_{0}$ if $v \in$ $\sigma \notin \mathcal{Q}_{0}$, or $(\mathcal{S}(\mathcal{Q}, v))_{0}=\mathcal{S}\left(\mathcal{Q}_{0}, v\right)$ otherwise, along with the fact that $\operatorname{NS}^{\prime}(\mathcal{Q})$ depends only on the set of fully non-simplicial monoids, independent of any choice of order.

The following is an immediate corollary of Proposition 5.2 and Theorem 5.4 .

Corollary 5.5. Let $\mathcal{R}_{i} i=1,2$, be refinements of $\mathcal{Q}$. Then a mutual smooth refinement exists, namely $\mathrm{NS}\left(\mathcal{R}_{1} \times{ }_{\mathcal{Q}} \mathcal{R}_{2}\right) \longrightarrow \mathcal{Q}$.

Finally, we include here a lemma which will be of use in Section 9 .

Lemma 5.6. Let $\mathcal{Q}_{0} \subset \mathcal{Q}$ be a monoidal subcomplex and $\mathcal{R}_{0} \longrightarrow \mathcal{Q}_{0}$ a refinement. Then there exists a refinement $\mathcal{R} \longrightarrow \mathcal{Q}$ which extends $\mathcal{R}_{0}$, meaning that it contains $\mathcal{R}_{0}$ as a subcomplex. If $\mathcal{R}_{0}$ is smooth, then a smooth extension exists.

Proof. Say a monoid $\tau \in \mathcal{Q}$ is "refined by $\mathcal{R}_{0}$ " if $\tau \in \mathcal{Q}_{0}$ and $\mathcal{R}_{0}(\tau) \longrightarrow \tau$ is a non-trivial refinement (i.e. $\mathcal{R}_{0}(\tau) \neq \tau$ ). We then say that $\sigma \in \mathcal{Q}$ is "damaged by 
$\mathcal{R}_{0}$ " if some face $\tau \leq \sigma$ is refined by $\mathcal{R}_{0}$, but $\sigma$ itself is not. In particular, note that if $\sigma$ is damaged, then $\sigma \notin \mathcal{Q}_{0}$; otherwise $\mathcal{R}_{0} \longrightarrow \mathcal{Q}_{0}$ would fail to be a refinement.

If there are no monoids which are damaged by $\mathcal{R}_{0}$, then

$$
\mathcal{R}:=\mathcal{R}_{0} \cup \mathcal{Q} \backslash \mathcal{Q}_{0} \longrightarrow \mathcal{Q}
$$

is an extension of $\mathcal{R}_{0}$.

Let $d\left(\mathcal{Q}, \mathcal{R}_{0}\right)$ denote the minimum dimension of $\sigma \in \mathcal{Q}$ such that $\sigma$ is damaged by $\mathcal{R}_{0}$. We will produce a refinement $\mathcal{R}_{1}$ of a subcomplex $\mathcal{Q}_{1} \subset \mathcal{Q}$ which extends $\mathcal{R}_{0}$ and for which $d\left(\mathcal{Q}, \mathcal{R}_{1}\right)>d\left(\mathcal{Q}, \mathcal{R}_{0}\right)$. Proceeding by induction, we eventually obtain a refinement $\mathcal{R}_{k}$ of $\mathcal{Q}_{k} \subset \mathcal{Q}$ extending $\mathcal{R}_{0}$ which damages no monoids on $\mathcal{Q}$, since $d\left(\mathcal{Q}, \mathcal{R}_{k}\right)$ is bounded by the maximum dimension of a monoid in $\mathcal{Q}$, and then we can take $\mathcal{R}=\mathcal{R}_{k} \cup \mathcal{Q} \backslash \mathcal{Q}_{k}$ as above.

For the induction, assume a refinement $\mathcal{R}_{j-1} \longrightarrow \mathcal{Q}_{j-1} \subset \mathcal{Q}$ is given which extends $\mathcal{R}_{0}$. Let $\mathcal{Q}_{j}$ be the subcomplex of $\mathcal{Q}$ consisting of $\mathcal{Q}_{j-1}$ and all monoids of dimension $d\left(\mathcal{Q}, \mathcal{R}_{j-1}\right)$ which are damaged by $\mathcal{R}_{j-1}$ along with their faces. Note that none of their proper faces are damaged by definition of $d\left(\mathcal{Q}, \mathcal{R}_{j-1}\right)$. Let $\Lambda_{j}$ be the set of all monoids of the form $\tau+\mathbb{N} v$, where $v=v_{1}+\cdots+v_{n}$ is the sum over extremals of a damaged monoid $\sigma$ in $\mathcal{Q}_{j}$, and either

(i) $\tau \leq \sigma$, such that $\tau \notin \mathcal{Q}_{j-1}$, or

(ii) $\tau \in \mathcal{R}_{j-1}\left(\tau^{\prime}\right)$ for some $\tau^{\prime} \leq \sigma$.

Then set

$$
\mathcal{R}_{j}=\mathcal{R}_{j-1} \cup \Lambda_{j}
$$

We claim $\mathcal{R}_{j}$ refines $\mathcal{Q}_{j}$ and $d\left(\mathcal{Q}, \mathcal{R}_{j}\right)>d\left(\mathcal{Q}, \mathcal{R}_{j-1}\right)$.

Indeed, identifying monoids in $\mathcal{R}_{j}$ with their images in monoids in $\mathcal{Q}$, the intersection of a monoid in $\mathcal{R}_{j-1}$ and one in $\Lambda_{j}$ must be a face of each in $\mathcal{R}_{j-1}$; the intersection of two monoids in $\Lambda_{j}$ is a face of each in $\Lambda_{j}$; and it is clear that the support of any $\sigma \in \mathcal{Q}_{j}$ is covered by the supports of monoids in $\mathcal{R}_{j}$, so $\mathcal{R}_{j} \longrightarrow \mathcal{Q}_{j}$ is a refinement.

For the second claim, suppose there was a monoid $\sigma \in \mathcal{Q}$ with $\operatorname{dim}(\sigma) \leq$ $d\left(\mathcal{Q}, \mathcal{R}_{j-1}\right)$ which was damaged by $\mathcal{R}_{j}$. As noted above, $\sigma \notin \mathcal{Q}_{j}$, which means that $\sigma$ is not damaged by $\mathcal{R}_{j-1}$ and therefore must be damaged by $\mathcal{R}_{j} \backslash \mathcal{R}_{j-1}=\Lambda_{j}$. In other words, $\sigma$ has a proper face $\tau$ which is non-trivially refined by $\Lambda_{j}$, but then $\tau \in \mathcal{Q}_{j}$ and $\operatorname{dim}(\sigma)>\operatorname{dim}(\tau)=d\left(\mathcal{Q}, \mathcal{R}_{j-1}\right)$, a contradiction.

Finally, if $\mathcal{R}_{0}$ is smooth, then we can replace $\mathcal{R}$ by $\mathrm{NS}(\mathcal{R})$ since $(\mathrm{NS}(\mathcal{R}))_{0}=$ $\operatorname{NS}\left(\mathcal{R}_{0}\right)=\mathcal{R}_{0}$ by Theorem 5.4 .

\section{Generalized BlOW-Up of A MANifold With CORNERS}

We first describe a functor $X \longmapsto \mathcal{P}_{X}$ which assigns to a manifold with corners its 'basic smooth monoidal complex' over the poset of the boundary faces of $X$ and also assigns to a b-map $f: X \longrightarrow Y$ a morphism $f_{\natural}: \mathcal{P}_{X} \longrightarrow \mathcal{P}_{Y}$. Next we show that for any smooth refinement $\mathcal{R} \longrightarrow \mathcal{P}_{X}$, the local construction of a generalized blow-up in Section 3 extends to give a new manifold with corners $[X ; \mathcal{R}]$ with a basic complex realizing $\mathcal{R}$.

Let $X$ be a compact manifold with corners, and consider the set $\mathcal{M}(X)=$ $\bigcup_{k=0}^{n} \mathcal{M}_{k}(X)$ of its boundary faces. It is partially ordered with respect to inclusion, but here we will equip it with the reverse $\operatorname{order}(\mathcal{M}(X), \leq)$, in which

$$
(\mathcal{M}(X), \leq) \ni G \leq F \Longleftrightarrow G \supseteq F .
$$


Definition 6.1 (Basic monoidal complex). The basic monoidal complex of $X$, denoted $\mathcal{P}_{X}$, consists of the smooth monoids

$$
\sigma_{F}=\bigoplus_{\substack{G \in \mathcal{M}_{1}(X) \\ F \leq G}} \mathbb{N} e_{G}, \quad F \in \mathcal{M}(X),
$$

freely generated by the boundary hypersurfaces containing a given face, with the obvious morphisms

$$
\sigma_{F^{\prime}} \hookrightarrow \sigma_{F}, \quad F^{\prime} \leq F \in \mathcal{M}(X) .
$$

It is smooth, complete and reduced, and indexed by the poset $(\mathcal{M}(X), \leq)$.

If $f: X \longrightarrow Y$ is a b-map, then

$$
f_{\#}:(\mathcal{M}(X), \leq) \longrightarrow(\mathcal{M}(Y), \leq)
$$

is order preserving, and $f$ induces a morphism of monoidal complexes

$$
f_{\natural}: \mathcal{P}_{X} \longrightarrow \mathcal{P}_{Y}
$$

where $f_{\natural}: \sigma_{F} \longrightarrow \sigma_{f_{\#}(F)}$ is generated by the boundary exponents (1.4) of $f$ :

$$
\begin{gathered}
f_{\natural}: \mathbb{N}\left\langle e_{G_{1}}, \ldots, e_{G_{k}}\right\rangle \longrightarrow \mathbb{N}\left\langle e_{H_{1}}, \ldots, e_{H_{l}}\right\rangle, \\
e_{G_{i}} \longmapsto \sum_{j} \alpha\left(G_{i}, H_{j}\right) e_{H_{j}} .
\end{gathered}
$$

The complex $\mathcal{P}_{X}$ has a canonical embedding into global sections of the b-normal bundles over the faces of $X$ as follows. If $F \in \mathcal{M}_{k}(X)$, recall that ${ }^{\mathrm{b}} N F \longrightarrow F$ is canonically trivialized by the frame $\left(x_{1} \partial_{x_{1}}, \ldots, x_{k} \partial_{x_{k}}\right)$, where $x_{i}$ are any boundary defining functions for the hypersurfaces $G_{i}$ containing $F$. These elements are well defined independent of the choices of the $x_{i}$, and they therefore generate a smooth monoid which may be identified with $\sigma_{F}$ :

$$
\sigma_{F} \cong \mathbb{N}\left\langle x_{1} \partial_{x_{1}}, \ldots, x_{k} \partial_{x_{k}}\right\rangle \subset{ }^{\mathrm{b}} N F, \quad F \in \mathcal{M}(X) .
$$

If $F \subseteq F^{\prime}$, then ${ }^{\mathrm{b}} N F^{\prime}$ is trivialized by a frame $\left(x_{i} \partial_{x_{i}}\right)_{i \in I}, I \subset\{1, \ldots, k\}$, and at any $p \in F$ there is a natural inclusion ${ }^{\mathrm{b}} N_{p} F^{\prime} \subset{ }^{\mathrm{b}} N_{p} F$ which induces over $F^{\prime} \leq F$ the natural homomorphism

$$
i_{F^{\prime} F}: \sigma_{F^{\prime}} \cong \mathbb{N}\left\langle x_{i} \partial_{x_{i}}\right\rangle_{i \in I} \hookrightarrow \sigma_{F} \cong \mathbb{N}\left\langle x_{1} \partial_{x_{1}}, \ldots, x_{k} \partial_{x_{k}}\right\rangle,
$$

which is an isomorphism onto the corresponding face of $\sigma_{F}$ and agrees with the previous definition. Furthermore, if $f: X \longrightarrow Y$ is a b-map, then under this identification (6.4) is given by the b-differential

$$
f_{\natural}={ }^{\mathrm{b}} f_{*}: \sigma_{F} \subset{ }^{\mathrm{b}} N F \longrightarrow \sigma_{f_{\#}(F)} \subset{ }^{\mathrm{b}} N f_{\#}(F)
$$

since the latter is integral with respect to the bases $\left\{x_{i} \partial_{x_{i}}\right\}$ for ${ }^{\mathrm{b}} N F$ and $\left\{x_{j}^{\prime} \partial_{x_{j}^{\prime}}\right\}$ for ${ }^{\mathrm{b}} N f_{\#}(F)$, and ${ }^{\mathrm{b}} f_{*}$ intertwines the inclusion ${ }^{\mathrm{b}} N F^{\prime} \subseteq{ }^{\mathrm{b}} N F$ (generating the face map $\left.i_{F^{\prime} F}\right)$ with the corresponding inclusion ${ }^{\mathrm{b}} N f_{\#}\left(F^{\prime}\right) \subseteq{ }^{\mathrm{b}} N f_{\#}(F)$.

Hereafter we will identify $\mathcal{P}_{X}$ with its image in the b-normal spaces of $X$.

Theorem 6.2 (Generalized blow-up of a manifold). If $X$ is a manifold with corners, then any smooth refinement, $\mathcal{R}$ of $\mathcal{P}_{X}$, defines a manifold $[X ; \mathcal{R}]$, the generalized blow-up of $X$ with respect to $\mathcal{R}$, equipped with a blow-down map

$$
\beta:[X ; \mathcal{R}] \longrightarrow X,
$$


restricting to a diffeomorphism of the interiors and such that $\beta_{\sharp}: \mathcal{P}_{[X ; \mathcal{R}]} \longrightarrow \mathcal{P}_{X}$ factors through an isomorphism

$$
\mathcal{P}_{[X ; \mathcal{R}]} \stackrel{\cong}{\longrightarrow} \mathcal{R}
$$

of monoidal complexes.

In particular, $\mathcal{M}([X ; \mathcal{R}])$ is determined as a poset by $\mathcal{R}$, so $[X ; \mathcal{R}]$ has a unique boundary face $F_{\tau}$ for each $\tau \in \mathcal{R}$, with $\operatorname{codim}\left(F_{\tau}\right)=\operatorname{dim}(\tau)$ and

$$
F_{\sigma} \subseteq F_{\tau} \Longleftrightarrow \tau \leq \sigma .
$$

Proof. As a manifold $X=\bigcup W_{i}$ has a locally finite open covering by coordinate charts $\phi_{i}: W_{i} \cong V_{i} \subset \mathbb{R}_{+}^{k(i)} \times \mathbb{R}^{n-k(i)}$. It can further be arranged that the cover is 'good' in the sense that all intersections of the coordinate sets are contractible and that the origin is in the image of each coordinate chart, so that the codimension $k(i)$ is achieved. Then the image of each coordinate chart is actually diffeomorphic to $\mathbb{R}_{+}^{k(i)} \times \mathbb{R}^{n-k(i)}$, so, by composing with such a diffeomorphism, it can be assumed that each coordinate chart is surjective. The manifold is then recovered, up to global diffeomorphism, by gluing

$$
X \cong \bigsqcup_{i} V_{i} / \sim,
$$

where the equivalence relation is generated by the transition maps: $p \sim q$ if and only if $f_{i j}(p)=q$ for some $i$ and $j$. Here, $f_{i j}=\phi_{j} \circ \phi_{i}^{-1}: O_{i j} \longrightarrow O_{j i}$ is a diffeomorphism on the sets $O_{i j}=\phi_{i}\left(W_{i} \cap W_{j}\right)$ whenever $W_{i} \cap W_{j} \neq \emptyset$.

In essence the blown-up manifold is obtained by blowing up each coordinate chart and showing that the transition maps lift to be smooth.

For each $i$, let $F_{i} \in \mathcal{M}_{k(i)}(X)$ be the unique boundary face of (maximal) codimension $k(i)$ such that $F_{i} \cap W_{i} \neq \emptyset$ and let $\mathcal{R}\left(F_{i}\right)=\mathcal{R}\left(\sigma_{F_{i}}\right)$ be the induced refinement of $\sigma_{F_{i}}$, interpreted as a refinement of the basic monoid $\sigma_{F_{i}} \cong \sigma_{\mathbb{R}_{+}^{n}}$ of $\mathbb{R}_{+}^{n}$. Whenever $W_{i} \cap W_{j} \neq \emptyset$, there is a unique smallest boundary face $G_{i j} \in \mathcal{M}(X)$ which meets $O_{i j}$ and contains both $F_{i}$ and $F_{j}$, so that $\mathcal{R}\left(F_{i}\right)=\left(\mathcal{R}\left(G_{i j}\right)\right)\left(\sigma_{F_{i}}\right)$. Then set

$$
V_{i}^{\prime}=\left[\mathbb{R}_{+}^{k(i)} ; \mathcal{R}\left(F_{i}\right)\right] \times \mathbb{R}^{n-k(i)},
$$

and for each pair $(i, j)$, set

$$
O_{i j}^{\prime}=\left(\beta_{i} \times \mathrm{Id}\right)^{-1}\left(O_{i j}\right) \subset\left[\mathbb{R}_{+}^{k(i j)} ; \mathcal{R}\left(G_{i j}\right)\right] \times \mathbb{R}^{n-k(i j)} \subset\left[\mathbb{R}_{+}^{k(i)} ; \mathcal{R}\left(F_{i}\right)\right] \times \mathbb{R}^{n-k(i)} .
$$

In light of Corollary 3.7, there are unique diffeomorphisms

$$
f_{i j}^{\prime}: O_{i j}^{\prime} \stackrel{\cong}{\longrightarrow} O_{j i}^{\prime}
$$

lifting $f_{i j}$, and we can therefore define

$$
[X ; \mathcal{R}]=\bigsqcup_{i} V_{i}^{\prime} / \sim
$$

where $p \sim q \Longleftrightarrow f_{i j}^{\prime}(p)=q$ for some pair $(i, j)$.

Since they commute with the transition maps, $f_{i j}^{\prime}$, the local blow-down maps $\beta_{i}: V_{i}^{\prime} \longrightarrow V_{i}$ patch together to define the global blow-down map

$$
\beta:[X ; \mathcal{R}] \longrightarrow X \text {. }
$$

Clearly $[X ; \mathcal{R}]$ is paracompact. To verify that it is Hausdorff, let $p$ and $q$ be distinct points. If $\beta(p) \neq \beta(q)$, then they can be separated by sets of the form 
$\beta^{-1}\left(O_{i}\right)$, where $O_{i}$ for $i=1,2$ are open sets in $X$ separating $\beta(p)$ and $\beta(q)$. On the other hand, if $\beta(p)=\beta(q)$, then $p$ and $q$ can be separated inside some set $V_{i}^{\prime}$, as in the proof of Proposition 3.1

For a fixed $F \in \mathcal{M}(X)$, it follows from Proposition 3.1 that each $V_{i}^{\prime}$ for which $F_{i}=F$ has boundary faces $\left(F_{i}\right)_{\sigma}$ in correspondence with monoids $\sigma \in \mathcal{R}(F)$. These are connected in $[X ; \mathcal{R}]$ for adjacent pairs such that $V_{i} \cap V_{j} \cap F \neq \emptyset$ since the diffeomorphisms $f_{i j}^{\prime}$ preserve the identification of boundary hypersurfaces with monoids in $\mathcal{R}(F)$. Thus for each $\sigma \in \mathcal{R}$ there is a unique boundary face $F_{\sigma} \in$ $\mathcal{M}([X ; \mathcal{R}])$ given by the quotient of the union of the local boundary faces $\left(F_{i}\right)_{\sigma}$, and hence the blow-down maps give an identification

$$
\mathcal{P}_{[X ; \mathcal{R}]} \cong \mathcal{R} \text {. }
$$

In fact, the blow-up can be defined globally near each boundary face since $F \in$ $\mathcal{M}_{k}(X)$ has a neighborhood in $X$ which is diffeomorphic to $\mathbb{R}_{+}^{k} \times F$, and then the preimage of this open set in $[X ; \mathcal{R}]$ is diffeomorphic to $\left[\mathbb{R}_{+}^{k} ; \mathcal{R}(F)\right] \times F$ obtained by localizing the resolution to boundary faces containing $F$.

If $f: X \longrightarrow Y$ is a b-map and $\beta:[Y ; \mathcal{R}] \longrightarrow Y$ is a generalized blow-down map, we say $f$ is compatible with $\beta$ if the morphism $f_{\natural}: \mathcal{P}_{X} \longrightarrow \mathcal{P}_{Y}$ factors through a morphism $\phi: \mathcal{P}_{X} \longrightarrow \mathcal{R}$ :

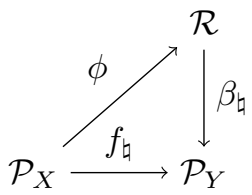

Theorem 6.3 (Lifting b-maps). If $f: X \longrightarrow Y$ is an interior b-map compatible with a generalized blow-up in the sense of (6.5), then $f$ lifts uniquely to a b-map $f^{\prime}: X \longrightarrow[Y ; \mathcal{R}]$ such that

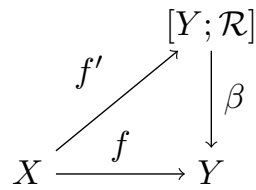

commutes and such that $f_{\natural}^{\prime}=\phi: \mathcal{P}_{X} \longrightarrow \mathcal{R} \cong \mathcal{P}_{[Y ; \mathcal{R}]}$.

Proof. Again, the construction is local. We consider a covering of $Y$ by coordinate charts $V_{i} \cong \mathbb{R}_{+}^{k(i)} \times \mathbb{R}^{n-k(i)}$ and, refining if necessary, a covering of $X$ by coordinate charts $W_{j} \cong \mathbb{R}_{+}^{l(j)} \times \mathbb{R}^{m-l(j)}$ such that for all $j, f\left(W_{j}\right) \subset V_{i}$ for some $i$. As in the previous proof, for each $i$ there is a maximal codimension face $F_{i} \in \mathcal{M}_{k(i)}(Y)$ such that $F_{i} \cap V_{i} \neq \emptyset$, and similarly for each $j$ a face $G_{j} \in \mathcal{M}_{l(j)}(X)$ such that $W_{j} \cap G_{j} \neq \emptyset$. Shrinking the coordinate charts if necessary, we can assume without loss of generality that $f_{\#}\left(G_{j}\right)=F_{i}$.

Locally, $f$ has the form

$$
f_{j}=f_{\mid W_{j}}: \mathbb{R}_{+}^{l(j)} \times \mathbb{R}^{m-l(j)} \longrightarrow \mathbb{R}_{+}^{k(i)} \times \mathbb{R}^{n-k(i)}
$$

which lifts by Proposition 3.6 to a b-map

$$
f_{j}^{\prime}: W_{j} \cong \mathbb{R}_{+}^{l(j)} \times \mathbb{R}^{m-l(j)} \longrightarrow V_{i}^{\prime} \cong\left[\mathbb{R}_{+}^{k(i)} ; \mathcal{R}\left(F_{i}\right)\right] \times \mathbb{R}^{n-k(i)} .
$$


It follows from the functoriality of these local lifted maps that they are compatible with the transition maps $g_{i j}^{\prime}: O_{i j}^{\prime} \longrightarrow O_{j i}^{\prime}$ used to construct $[Y ; \mathcal{R}]$, and so the $f_{j}^{\prime}$ patch together to form a b-map

$$
f^{\prime}: X \longrightarrow[Y ; \mathcal{R}]
$$

as claimed.

Corollary 6.4. If $\mathcal{R}_{i}, i=1,2$, are two refinements of $\mathcal{P}_{X}$, then $\left[X ; \mathcal{R}_{1}\right] \cong\left[X ; \mathcal{R}_{2}\right]$ over $X$ if and only if $\mathcal{R}_{1} \cong \mathcal{R}_{2}$ as refinements of $\mathcal{P}_{X}$.

Proof. The 'if' direction is clear. For the converse, suppose $\phi:\left[X ; \mathcal{R}_{1}\right] \longrightarrow\left[X ; \mathcal{R}_{2}\right]$ is a diffeomorphism (which is necessarily a b-map) which intertwines the blow-down maps to $X$. Since $P_{\left[X ; \mathcal{R}_{i}\right]}=\mathcal{R}_{i}$ it follows that $\phi_{\#}: \mathcal{R}_{1} \longrightarrow \mathcal{R}_{2}$ is an isomorphism of complexes which intertwines the refinement morphisms $\mathcal{R}_{i} \longrightarrow \mathcal{P}_{X}$.

Even if $f: X \longrightarrow Y$ is not necessarily compatible with the refinement giving the blow-up $[Y ; \mathcal{R}]$, there are generalized blow-ups of $X$ through which $f$ does lift. Indeed, from Theorem 6.3 above a generalized blow-up $[X ; \mathcal{S}]$ admits a map to $[Y ; \mathcal{R}]$ over $f: X \longrightarrow Y$ precisely when $f_{\natural} \circ \beta_{\natural}: \mathcal{S} \longrightarrow \mathcal{P}_{Y}$ factors through $\mathcal{R} \longrightarrow \mathcal{P}_{Y}$. Such a blow-up always exists.

Theorem 6.5 (Blowing up the domain). Let $f: X \longrightarrow Y$ be an interior $b$-map and $[Y ; \mathcal{R}] \longrightarrow Y$ a generalized blow-up. Then there exists a generalized blow-up $[X ; \mathcal{S}] \longrightarrow X$ and a map $f^{\prime}:[X ; \mathcal{S}] \longrightarrow[Y ; \mathcal{R}]$ such that

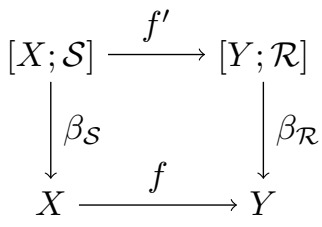

commutes.

Proof. First we consider $\mathcal{P}_{X} \times_{\mathcal{P}_{Y}} \mathcal{R} \longrightarrow \mathcal{P}_{X}$, which is a refinement by Proposition 5.3. If it is a smooth refinement, then we take $\mathcal{S}=\mathcal{P}_{X} \times_{\mathcal{P}_{Y}} \mathcal{R}$ and we are done. Note that in this case $[X ; \mathcal{S}]$ is the unique "minimal" blow-up, meaning it is universal among blow-ups of $X$ which lift $f$ : any other blow-up which lifts $f$ must factor through $[X ; \mathcal{S}]$ by the universality of fiber products of monoidal complexes and Theorem 6.3. In general however, $\mathcal{P}_{X} \times{ }_{\mathcal{P}_{Y}} \mathcal{R}$ is not smooth. We let $\mathcal{S}$ be any smooth refinement of $\mathcal{P}_{X} \times \mathcal{P}_{Y} \mathcal{R}$, for instance the natural smooth refinement $\operatorname{NS}\left(\mathcal{P}_{X} \times_{\mathcal{P}_{Y}} \mathcal{R}\right)$ of Theorem 5.4. There are many other choices, none of which is universal.

Note that this includes Theorem 6.3 as a special case, as follows from the fact that $f_{\natural}: \mathcal{P}_{X} \longrightarrow \mathcal{P}_{Y}$ is compatible with $\mathcal{R} \longrightarrow \mathcal{P}_{Y}$ if and only if $\mathcal{P}_{X} \times_{\mathcal{P}_{Y}} \mathcal{R} \longrightarrow \mathcal{P}_{X}$ is an isomorphism, which we leave as an exercise for the reader.

\section{ORDINARY BLOW-UP AND EXAMPLES}

Recall the ordinary blow-up of $[X ; F]$ of a boundary face $F \in \mathcal{M}_{k}(X)$. As a set this is

$$
[X ; F]=X \backslash F \cup S N_{+} F,
$$

where $S N_{+} F \stackrel{\pi}{\longrightarrow} F$ denotes the inward-pointing spherical normal bundle and the blow-down map $\beta:[X ; F] \longrightarrow X$ is given by the identity on $X \backslash F$ and by $\pi$ on 
$S N_{+} F$ (see Figure 4). The smooth structure on $[X ; F]$ is generated by $\beta^{*} C^{\infty}(X)$ as well as the quotients $x_{i} / x_{j}$ (where they are finite) of boundary defining functions for the boundary hypersurfaces $H_{i}$ such that $F$ is a component of $\bigcap_{i} H_{i}$.

Proposition 7.1. The ordinary blow-up of $X$ at the boundary face $F$ is the generalized blow-up corresponding to the star subdivision along the sum over generators of $\sigma_{F}$. That is,

$$
[X ; F] \cong\left[X ; \mathcal{S}\left(\mathcal{P}_{X}, v_{F}\right)\right]
$$

where $v_{F}=\sum_{i} x_{i} \partial_{x_{i}}$ and $\sigma_{F}=\mathbb{N}\left\langle x_{i} \partial_{x_{i}}\right\rangle_{1 \leq i \leq \operatorname{codim}(F)}$.

Proof. With the smooth structure above, the "front face" $S N_{+} F \in \mathcal{M}([X ; F])$ is a boundary hypersurface which is fibered over $F$, whose fiber is a $(k-1)$-simplex where $k=\operatorname{codim}(F)$. In fact, for any coordinate chart $\left(x, x^{\prime}, y\right): U \cong \mathbb{R}_{+}^{k} \times \mathbb{R}_{+}^{l-k} \times \mathbb{R}^{n-l}$ in $X$ such that $F \cap U \cong\left\{x_{1}=\cdots=x_{k}=0\right\}$, there are $k$ coordinate charts $\widetilde{U}_{i}$, $i=1, \ldots, k$, covering $\beta^{-1}(U)$ in $[X ; F]$ :

$$
\left(t_{i}, x^{\prime}, y\right): \widetilde{U}_{i} \cong \mathbb{R}_{+}^{k} \times \mathbb{R}_{+}^{l-k} \times \mathbb{R}^{n-l}
$$

where

$$
t_{i, j}= \begin{cases}x_{i} & \text { if } i=j \\ x_{j} / x_{i} & \text { otherwise }\end{cases}
$$

Thus $\beta: \widetilde{U}_{i} \longrightarrow U$ has the form

$$
\beta:\left(t_{i}, x^{\prime}, y\right) \longmapsto\left(t_{i}^{\mu_{i}}, x^{\prime}, y\right)=\left(x, x^{\prime}, y\right)
$$

where $\mu_{i}$ is the identity matrix with its $i$ th row replaced by ones:

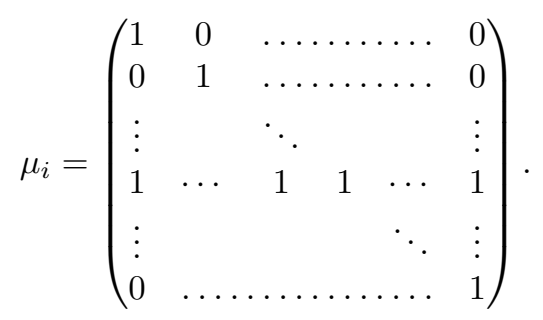

Observe that $\mu_{i}^{\mathrm{T}}$ represents the monoid inclusion

$$
\mathbb{N}\left\langle x_{1} \partial_{x_{1}}, \ldots, x_{i-1} \partial_{x_{i-1}}, v_{F}, \ldots, x_{k} \partial_{x_{k}}\right\rangle \longleftrightarrow \mathbb{N}\left\langle x_{1} \partial_{x_{1}}, \ldots, x_{k} \partial_{x_{k}}\right\rangle,
$$

where $v_{F}=x_{1} \partial_{x_{1}}+\cdots+x_{k} \partial_{x_{k}}$. The collection of these $\mu_{i}^{\mathrm{T}}, i=1, \ldots, k$, therefore gives the monoid homomorphisms from the maximal dimension $\tau \in \mathcal{S}\left(\mathcal{P}, v_{F}\right)\left(\sigma_{F}\right)$ to $\sigma_{F}$.

All coordinate charts meeting $F$ are blown up in this way, according to the star subdivision $\mathcal{S}\left(\mathcal{P}_{X}, v_{F}\right)$, and this is precisely the construction of the generalized blow-up $\left[X ; \mathcal{S}\left(\mathcal{P}_{X}, v_{F}\right)\right] \longrightarrow X$.

The inhomogeneous blow-up of $F \in \mathcal{M}(X)$ is similar to ordinary blow-up but is more general. It consists again of the set $X \backslash F \cup S N_{+} F$, but the smooth structure is generated over $\beta^{*} C^{\infty}(X)$ by quotients of the form $x_{i}^{1 / n(i)} / x_{j}^{1 / n(j)}$, where the $n(i) \in \mathbb{N}$ are consistently associated with the boundary hypersurfaces through $F$.

The proof of Proposition 7.1 can be modified in a straightforward manner to give the following result. 


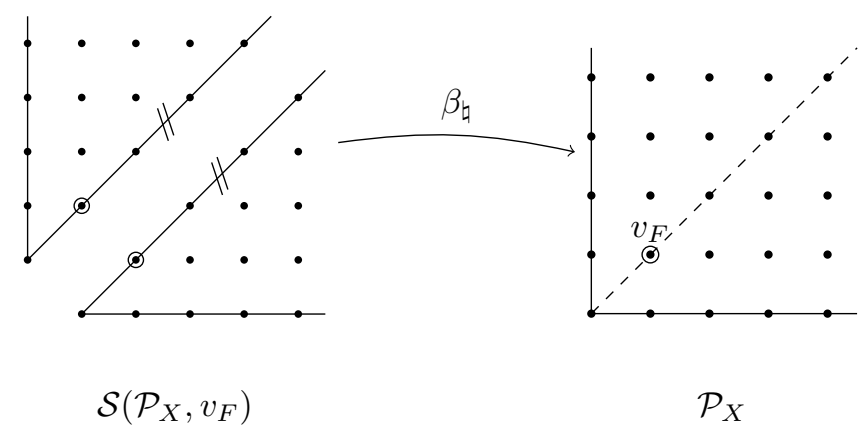

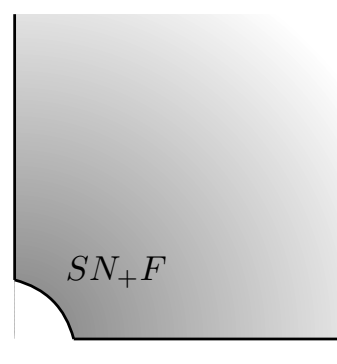

$[X ; F]$

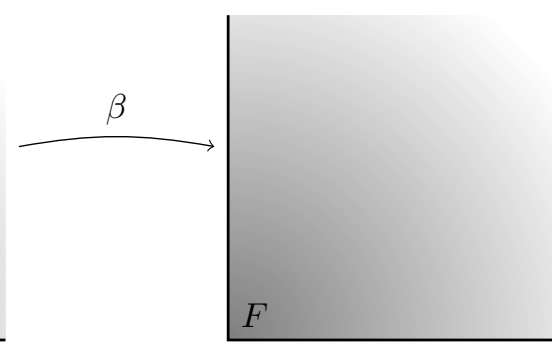

$X$

Figure 4. The ordinary blow-up of $F$ in $X$, and the associated star subdivision. Only the monoids of maximal dimension are pictured.

Proposition 7.2. Let $n:\left\{H \in \mathcal{M}_{1}(X) ; F \subseteq H\right\} \longrightarrow \mathbb{N}$ be an assignment of integer roots to the boundary hypersurfaces through $F$. Then the inhomogeneous blow-up of $F$ with respect to $n$ is realized by the generalized blow-up by the weighted star subdivision $\left[X ; \mathcal{S}\left(\mathcal{P}_{X}, v_{F, n}\right)\right]$, where

$$
v_{F, n}=\sum_{i} n(i) x_{i} \partial_{x_{i}}
$$

is the corresponding weighted sum of the generators of $\sigma_{F}=\mathbb{N}\left\langle x_{1} \partial_{x_{1}}, \ldots, x_{n} \partial_{x_{n}}\right\rangle$.

Iterating either of these constructions, we find that iterated boundary blow-up is also a special case of generalized blow-up. Recall that the lift (or proper transform) of a submanifold $Y \subset X$ under a blow-up $\beta:[X ; F] \longrightarrow X$ is the set

$$
\beta^{\#}(Y)= \begin{cases}\beta^{-1}(Y) & \text { if } Y \subseteq F, \\ \operatorname{clos}\left(\beta^{-1}(Y \backslash F)\right), & \text { otherwise. }\end{cases}
$$

The iterated boundary blow-up $\left[X ; F_{N}, \ldots, F_{1}\right], F_{i} \in \mathcal{M}(X)$, is defined by successive lifting:

$$
\left[X ; F_{1}, \ldots, F_{N}\right]=\left[\cdots\left[\left[X ; F_{1}\right], \beta_{1}^{\#}\left(F_{2}\right)\right], \cdots, \beta_{1}^{\#} \circ \cdots \circ \beta_{N-1}^{\#}\left(F_{N}\right)\right],
$$

where $\beta_{i}:\left[X ; F_{1}, \ldots, F_{i}\right] \longrightarrow\left[X ; F_{1}, \ldots, F_{i-1}\right]$, and can be extended similarly to the case of inhomogeneous blow-up. 
Observe that the lift of a boundary face $G \in \mathcal{M}(X)$ to the blow-up $\beta:[X ; F] \longrightarrow$ $X$ is again a boundary face of $[X ; F]$. In light of the identification of boundary faces of a generalized blow-up with monoids in the refinement, it follows directly that iterated boundary blow-up is realized by iterated star subdivisions.

Corollary 7.3. Let $F_{1}, \ldots, F_{N} \in \mathcal{M}(X)$, and let $\mathcal{R}_{1}, \ldots, \mathcal{R}_{N}$ be the sequence of refinements of $\mathcal{P}_{X}$ obtained by iteratively defining $\mathcal{R}_{j}=\mathcal{S}\left(\mathcal{R}_{j-1}, v_{\widetilde{F}_{j}}\right)$, where $\widetilde{F}_{j}=\beta_{1}^{\#} \circ \cdots \circ \beta_{j-1}^{\#}\left(F_{j}\right)$ :

$$
\left[X ; F_{N}, \ldots, F_{1}\right]=\left[X ; \mathcal{R}_{N}\right] .
$$

One might wonder at this point if there are generalized blow-ups of $X$ which are not of the above type. In fact we will prove in Section 8 that generalized blow-ups are determined uniquely up to diffeomorphism by their refinements. It follows that any smooth refinement of $\mathcal{P}_{X}$ which cannot be obtained by iterated (possibly weighted) star subdivision gives a generalized blow-up which is not of this classical type. Examples of these are easy to construct provided $X$ has corners with codimension at least 3 .

\section{Characterization of generalized Blow-DOWn maps}

In this section, which is independent from the remainder of the paper, we complete our treatment of generalized blow-up, showing that blow-down maps from a generalized blow-up of the target are characterized analytically among b-maps in general by two properties.

Definition 8.1. A generalized blow-down map between manifolds with corners, $\beta: X \longrightarrow Y$, is a proper b-map which is a diffeomorphism of the interiors and which has b-differential, ${ }^{\mathrm{b}} \beta_{*}:{ }^{\mathrm{b}} T_{x} X \longrightarrow{ }^{\mathrm{b}} T_{\beta(x)} Y$, an isomorphism for each $x \in X$.

We prove below that such a map induces a smooth refinement as its associated morphism of monoidal complexes.

The range of a continuous proper map is closed, so it follows directly from the definition that a generalized blow-down map is surjective. It is convenient, and there is no restriction, to assume in the subsequent discussion that $X$, and hence $Y$, is connected. We continue to follow the convention for local coordinates $(x, y)$, where $x_{i} \in \mathbb{R}_{+}$are local boundary defining functions and $y_{i} \in \mathbb{R}$ are tangential variables.

First we find a local normal form for a generalized blow-down map.

Lemma 8.2. Let $\beta: X \longrightarrow Y$ be a generalized blow-down map between compact manifolds, $p \in G \backslash \partial G$ a point in the interior of a boundary face $G \in \mathcal{M}_{k}(X)$, and $\left(x^{\prime}, y^{\prime}\right)=\left(x_{1}^{\prime}, \ldots, x_{k^{\prime}}^{\prime}, y_{1}^{\prime}, \ldots, y_{n-k^{\prime}}^{\prime}\right)$ local coordinates near $q=f(p) \in F=$ $\beta_{\#}(G) \in \mathcal{M}_{k^{\prime}}(Y)$. Then there exist local coordinates $(x, y, z)$ near $p$, with the $z_{i}>0$, such that, after perhaps renumbering the $x^{\prime}$ coordinates near $q, \beta$ has the local form

$$
\beta(x, z, y)=\left(x^{\nu_{1}}, \ldots, x^{\nu_{k}}, z_{1} x^{\nu_{k+1}}, \ldots, z_{k^{\prime}-k} x^{\nu_{k^{\prime}}}, y_{1}, \ldots, y_{n-k^{\prime}}\right)=\left(x^{\prime}, y^{\prime}\right),
$$

where the first $k$ of the $\nu_{i} \in \mathbb{Z}^{k}$ are linearly independent.

Proof. Let $\bar{x}_{j}, j=1, \ldots, k$, be local boundary defining functions for hypersurfaces through $p$. Then, since $\beta$ is an interior b-map,

$$
\beta^{*}\left(x_{i}^{\prime}\right)=a_{i} \bar{x}^{\nu_{i}}=a_{i} \prod_{j=1}^{k} \bar{x}_{j}^{\nu_{j i}}, 0<a_{i} \in C^{\infty}(X),
$$


where $\nu_{j i} \in \mathbb{N}$. Indeed, $\nu^{\mathrm{T}} \in \operatorname{Mat}\left(k^{\prime} \times k, \mathbb{N}\right)$ is the matrix representing ${ }^{\mathrm{b}} \beta_{*}:{ }^{\mathrm{b}} N G \rightarrow$ ${ }^{\mathrm{b}} N F$ with respect to the bases $\left\{\bar{x}_{i} \partial_{\bar{x}_{i}}\right\}$ and $\left\{x_{j}^{\prime} \partial_{x_{j}^{\prime}}\right\}$.

Since $\nu$ must have full rank (or else ${ }^{\mathrm{b}} \beta_{*}$ could not be bijective), relabeling the $x_{i}^{\prime}$ appropriately ensures that the $k \times k$ matrix formed by the first $k$ entries in the $\nu_{j i}$ is invertible and these entries give the vectors $\nu_{i}:=\left(\nu_{1 i}, \ldots, \nu_{k i}\right)$ for $i=1, \ldots, k$.

Changing the $\bar{x}_{j}$ by positive smooth factors, $x_{j}=b_{j} \bar{x}_{j}, 0<b_{j} \in C^{\infty}(X)$, multiplies the coefficient functions $a_{i}$ in (8.2) by the monomials $b^{\nu_{i}}$. The independence of $\nu_{i}, i \leq k$, means that the $b_{j}$ can be chosen so each $a_{i} \equiv 1$ for $i \leq k$. This gives the first $k$ equations in (8.1).

The tangential coordinates $y_{i}^{\prime}$ pull back under $\beta$ to be smooth and independent at $p$, so we take $y_{i}=\beta^{*} y_{i}^{\prime}$ for $i=1, \ldots, n-k^{\prime}$, ensuring the last $n-k^{\prime}$ equations in (8.1) and without affecting the first $k$.

Finally, then consider the pull-back of the last $k^{\prime}-k$ boundary defining functions. The logarithmic differentials, ${ }^{\mathrm{b}} \beta^{*}\left(d x_{i}^{\prime} / x_{i}^{\prime}\right)$, must be independent at $p$ and be independent of the $d x_{j} / x_{j}, j=1, \ldots, k$, and $d y_{l}, l=1, \ldots, n-k^{\prime}$. In view of (8.2) this means precisely that the smooth differentials $d a_{i} / a_{i}$ must be linearly independent at $p$ for $i=k+1, \ldots, k^{\prime}$, and since the $a_{i}>0$ this in turn is equivalent to the independence of the corresponding $d a_{i}$, so $z_{i}:=a_{k-i}, i=1, \ldots, k-k^{\prime}$, can be introduced as additional tangential coordinates giving (8.1).

In fact, one can take the $x_{i}^{\prime}$ to be globally defined boundary defining functions on a neighborhood of $F \backslash \partial F$, and then it follows from the proof that the $x_{i}$ can be taken to be global on a neighborhood of $G \backslash \partial G$. It follows similarly that the $y_{i}$ and $z_{j}$ are globally defined on each fiber $\beta^{-1}\left(q^{\prime}\right) \cap G \backslash \partial G$ for $q^{\prime}$ in a neighborhood of $q$, and it follows that these fibers are contractible. In fact, more is true.

Lemma 8.3. The functions $z_{i}, i=1, \ldots, k^{\prime}-k$, in (8.1) are globally defined on the fibers $\beta^{-1}\left(q^{\prime}\right) \cap G \backslash \partial G$ for $q^{\prime}$ in a neighborhood of $q$, and the map

$$
z: \beta^{-1}(q) \cap G \backslash \partial G \longrightarrow(0, \infty)^{k^{\prime}-k}
$$

is surjective.

Proof. As noted, $z$ is a globally defined map on the fiber in light of (8.2) and the fact that $z_{i}=a_{k-i}$. Suppose then that (8.3) is not surjective, and consider a point in the closure of the image under $z$ of a component of $\beta^{-1}(q) \cap G \backslash \partial G$. By compactness of $G$, this must be the image of a point $p^{\prime}$ in $\partial G$, in the interior of a boundary face $H \subseteq G$ with $H \in \mathcal{M}_{r}(X)$, say. The assumption that $z$ is not surjective means that $z\left(p^{\prime}\right)$ lies inside $(0, \infty)^{k-k^{\prime}}$, so that $0<z_{i}\left(p^{\prime}\right)<\infty$ for all $i \leq k-k^{\prime}$.

By continuity $p^{\prime}$ is mapped by $\beta$ to $q$. Consider the construction of the $x_{i}$ and $z_{i}$ in the proof of Lemma 8.2, where we now begin with additional boundary defining functions $\bar{x}_{i}^{\prime}, i=k+1, \ldots, r \leq k^{\prime}$, for $H$. Equations (8.2) become

$$
\beta^{*}\left(x_{i}^{\prime}\right)=a_{i} \bar{x}^{\nu_{j}}=a_{i}^{\prime} \bar{x}^{\prime \gamma_{i}} \bar{x}^{\nu_{i}}, 0<a_{i}^{\prime},
$$

where not all the $\gamma_{j} \in \mathbb{N}^{r-k}$ can vanish without violating the isomorphism condition on ${ }^{\mathrm{b}} \beta_{*}$. It follows by going through the construction in the proof that $z_{i}=e_{i} \bar{x}_{i}{ }_{i}^{\mu}$, where $\mu_{i} \in \mathbb{Q}^{r}$ cannot all vanish (though $\mu_{j i}$ may be non-integral and/or negative since the $b_{j}$ may now involve the $\bar{x}_{i}^{\prime}$ ), so at least one $z_{i}$ tends to either zero or infinity at $H$, giving a contradiction. 
From the normal form (8.1) we derive the following path lifting result, which determines in which face $G \in \beta^{-1}(F)$ a lifted path will hit the boundary of $X$ from its b-tangent at $t=0$.

Lemma 8.4. Let $\beta: X \longrightarrow Y$ be a generalized blow-down, $G \in \mathcal{M}(X), F=$ $\beta_{\#}(G) \in \mathcal{M}(Y)$ and $q \in F \backslash \partial F$, with coordinates $\left(x^{\prime}, y^{\prime}\right)$ centered at $q$. If

$$
\gamma:[0, \epsilon) \ni t \longmapsto\left(t^{\kappa}, 0\right) \in Y
$$

is a path with endpoint at $q$ and initial b-tangent vector $\kappa=\sum \kappa_{i} x_{i}^{\prime} \partial_{x_{i}^{\prime}} \in{ }^{\mathrm{b}} N_{+} F$, with all $\kappa_{i}>0$, then the lift $\cos \left(\beta^{-1}(\gamma((0, \epsilon)))\right.$ of the image of $\gamma$ to $X$ meets $\beta^{-1}(q) \cap G \backslash \partial G$ if and only if there exists $\lambda=\sum_{i} \lambda_{i} x_{i} \partial_{x_{i}} \in{ }^{\mathrm{b}} N_{+} G$ such that ${ }^{\mathrm{b}} f_{*}(\lambda)=\kappa$.

Proof. Since $\beta$ is a diffeomorphism on the interiors, the smooth path $\gamma:(0, \epsilon) \longrightarrow$ $Y \backslash \partial Y$ lifts to $X \backslash \partial X$. If such a $\lambda \in{ }^{\mathrm{b}} N_{+} G$ does exist, then near a component of $\beta^{-1}(q) \cap G \backslash \partial G$ it follows directly that $\gamma$ has a lift

$$
[0, \epsilon) \ni t \longmapsto\left(t^{\lambda}, 0,1\right)=(x, y, z)
$$

extending to $t=0$, with endpoint $p=(0,0,1) \in G \backslash \partial G$. (Observe that $p$ lies in the domain of the coordinates $(x, y, z)$ in light of Lemma 8.3.)

If no such $\lambda$ exists, then every point $p$ of $\beta^{-1}(q) \cap G \backslash \partial G$ has a neighborhood

$$
D(\epsilon, p)=\left\{(x, y, z) ; x_{i}<\epsilon, i=1, \ldots, k,|y|<\epsilon\right\}, \epsilon>0,
$$

which does not meet the lift of $\gamma$ to $X \backslash \partial X$.

Indeed, the image of such a neighborhood under $\beta$ contains a point of $\gamma$ if and only if

$$
\left(t^{\kappa_{1}}, \ldots, t^{\kappa_{k^{\prime}}}, 0\right)=\left(x^{\nu_{1}}, \ldots, x^{\nu_{k}}, z_{1} x^{\nu_{k+1}}, \ldots, z_{k^{\prime}-k} x^{\nu_{k^{\prime}}}, y\right) .
$$

Taking the logarithm of the first $k$ conditions gives

$$
\kappa_{i} \log t=\sum_{j} \nu_{j i} \log x_{j}=\left({ }^{\mathrm{b}} \beta_{*} \log x\right)_{i}
$$

since $\nu^{\mathrm{T}}$ is the matrix representing ${ }^{\mathrm{b}} \beta_{*}$. Now, in $D(\epsilon, p)$, the vector $(\log x) \epsilon$ $(-A,-\infty)^{k}$ where $A=-\log \epsilon>0$. Thus, the right side of (8.4) lies in $-{ }^{\mathrm{b}} \beta_{*}\left({ }^{\mathrm{b}} N_{+} G\right)$, but by assumption $\kappa \notin{ }^{\mathrm{b}} \beta_{*}\left({ }^{\mathrm{b}} N_{+} G\right)$, so (8.4) can have no solution with $0<t<$ 1 .

In fact, though we do not use this directly below, Lemmas 8.2 and 8.4 show that for all $G$ such that $\beta_{\#}(G)=F$, the map $\beta: G \backslash \partial G \longrightarrow F \backslash \partial F$ is a fibration, with fibers diffeomorphic to $(0, \infty)^{k^{\prime}-k}$.

To see this note that since $\beta: G \longrightarrow F$ is an interior b-map it follows from (8.1) that

$$
\beta: G \backslash \partial G \longrightarrow F \backslash \partial F
$$

is a submersion. By compactness of $G$, any limit point of the range of 8.5) must be the image of a point in $G$, so it must be surjective, or else an interior point of $F$ would only meet the boundary of $G$, which is inconsistent with the defining properties of an interior b-map. By Lemma 8.2 it follows that $z: \beta^{-1}(q) \cap G \backslash$ $\partial G \longrightarrow(0, \infty)^{k^{\prime}-k}$ is a covering map, hence the fibers of (8.5) are a disjoint union of components diffeomorphic to the latter space. By Lemma 8.4 these fibers must be connected, since otherwise there would be multiple preimages of $\gamma(t)$ for small $t$. 
Proposition 8.5. If $\beta: X \longrightarrow Y$ is a generalized blow-down map, then the morphism of monoidal complexes

$$
\beta_{\natural}: \mathcal{P}_{X} \longrightarrow \mathcal{P}_{Y}
$$

is a smooth refinement.

Proof. Since ${ }^{\mathrm{b}} \beta_{*}$ is bijective, $\beta_{\natural}$ is necessarily injective, and it suffices to verify that the cones

$$
\left\{\operatorname{supp}\left(\beta_{\natural}\left(\sigma_{G}\right)\right)=\beta_{*}\left({ }^{\mathrm{b}} N_{+} G\right) ; \beta_{\#}(G)=F\right\} \subset \operatorname{supp}\left(\sigma_{F}\right)={ }^{\mathrm{b}} N_{+} F
$$

have union equal to ${ }^{\mathrm{b}} N_{+} F$ and have no common interior vectors.

First observe that the union of these cones is indeed ${ }^{\mathrm{b}} N_{+} F$. If not, the complement, which is open, would contain an interior point of ${ }^{\mathrm{b}} N_{+} F$. Thus, proceeding by contradiction, we can suppose that there is a vector $\kappa=\left(\kappa_{1}, \ldots, \kappa_{k}\right) \in{ }^{\mathrm{b}} N_{+} F$, with positive integer entries, which is disjoint from all the $\beta_{*}\left({ }^{\mathrm{b}} N_{+} G\right)$. By Lemma 8.4 there is a path $\gamma(t) \in Y$ with an initial b-differential equal to $\kappa$ and an endpoint at $q \in F \backslash \partial F$ whose lift to $X$ does not meet any $G$ with $\beta_{\#}(G)=F$. On the other hand, by the properness of $\beta$ there is a sequence $0<t_{j} \rightarrow 0$ such that the points $\beta^{-1}\left(\gamma\left(t_{j}\right)\right)$ converge in $X$. By continuity, the limit must be in $\beta^{-1}(q)$. However, since $q$ lies in the interior of $F$, such a point must lie in the interior of one of the $G$ with $\beta_{\#}(G)=F$, which is a contradiction.

Next consider two of the cones $\beta_{*}\left({ }^{\mathrm{b}} N_{+} G_{i}\right), i=1,2$, which contain an interior vector $\kappa$ of ${ }^{\mathrm{b}} N_{+} F$ in both their relative interiors, i.e. this point is the image of an interior vector $\lambda_{i}$ of each of the ${ }^{\mathrm{b}} N_{+} G_{i}$. Lemma 8.4 applies to both faces, hence the curve $\gamma$ with endpoint $q \in F \backslash \partial F$ and initial b-tangent vector $\kappa$ has a lift with endpoint in the interior of each boundary face. The assumption that $\beta$ is a diffeomorphism in the interior therefore ensures that these two boundary faces have interiors which intersect and that they are therefore equal.

We conclude that $\beta_{\natural}: \mathcal{P}_{X} \rightarrow \mathcal{P}_{Y}$ is a refinement, which must be smooth since each $\sigma_{G} \in \mathcal{P}_{X}$ is smooth.

Proposition 8.6. A generalized blow-down map $\beta: X \longrightarrow Y$ is a diffeomorphism if and only if

$$
\beta_{\natural}: \mathcal{P}_{X} \longrightarrow \mathcal{P}_{Y}
$$

is invertible.

Proof. That $\mathcal{P}_{Y} \cong \beta_{\natural}\left(\mathcal{P}_{X}\right)$ if $\beta$ is a diffeomorphism is clear, since $\beta_{\natural}^{-1}$ furnishes an inverse.

Assume then that $\beta_{\natural}: \mathcal{P}_{X} \cong \mathcal{P}_{Y}$. For any $F \in \mathcal{M}_{k^{\prime}}(Y)$, there is therefore a unique $G \in \mathcal{M}_{k}(X)$ with $\beta_{\#}(G)=F$ and $\beta_{\natural}: \sigma_{G} \cong \sigma_{F}$ (it follows that $k=k^{\prime}$ ). Since the $\nu_{j}, j=1, \ldots, k$, in Lemma 8.2 are precisely the coordinates for the generators of $\sigma_{G}$ in terms of those of $\sigma_{F}$, we can arrange that $\nu=\mathrm{Id}$, and there are therefore local coordinates as in (8.1) near each point in which $\beta=$ Id.

Theorem 8.7. If $\beta: X \longrightarrow Y$ is a generalized blow-down map, then

$$
X \cong\left[Y ; \mathcal{P}_{X}\right]
$$

with respect to the simplicial refinement $\beta_{\natural}: \mathcal{P}_{X} \longrightarrow \mathcal{P}_{Y}$. 
Proof. Let $Y_{0}=\left[Y ; \mathcal{P}_{X}\right]$, and let $\beta_{0}: Y_{0} \longrightarrow Y$ be the blow-down. The morphism $\beta_{\natural}: \mathcal{P}_{X} \longrightarrow \mathcal{P}_{Y}$ is tautologically compatible with the refinement $\mathcal{P}_{X} \longrightarrow \mathcal{P}_{Y}$, since $\beta_{\natural}$ factors through the identity morphism Id $: \mathcal{P}_{X} \longrightarrow \mathcal{P}_{X}$.

From Theorem 6.3 then, $\beta$ lifts to a b-map

$$
\beta^{\prime}: X \longrightarrow Y_{0}
$$

which is easily seen to be a generalized blow-down since $\beta: X \backslash \partial X \longrightarrow Y \backslash \partial Y$ factors through $\beta^{\prime}: X \backslash \partial X \longrightarrow Y_{0} \backslash \partial Y_{0}$, which must therefore be a diffeomorphism. Since the lifted map on monoidal complexes is just the identity,

$$
\beta_{\natural}^{\prime}=\mathrm{Id}: \mathcal{P}_{X} \longrightarrow \mathcal{P}_{X},
$$

$X \cong Y_{0}$ by Proposition 8.6 .

\section{BinOMial SUBVARIETIES}

We consider subvarieties of a manifold which near the boundary have the local form

$$
a_{i} x^{\alpha_{i}}=b_{i} x^{\beta_{i}}, y_{j}=0, a_{i}, b_{i}>0,
$$

which is to say they are given by the vanishing of some binomial equations with smooth positive coefficients in the boundary defining variables and by the vanishing of some interior variables. Such objects occur naturally in the setting of fiber products and in other contexts, for instance the embedding under a b-map of one manifold into another.

After verifying that such subvarieties are well defined and have a standard form, we show that, although they are not in general smoothly embedded manifolds with corners, they have enough structure to support the machinery of monoidal complexes and b-maps. This allows us to develop their resolution theory in the next section. While it would be possible to define an intrinsic category of abstract 'binomial varieties', since it suffices in our later treatment of fiber products, attention here is restricted to the case of a binomial variety explicitly embedded in a manifold.

Observe in (9.1) that by dividing by $b_{i} x^{\beta_{i}}$ and also exponentiating the interior coordinates, the equations take the unified form $a_{i}^{\prime} x^{\gamma_{i}}=1$, with $\gamma_{i}=\alpha_{i}-\beta_{i}$, $a_{i}^{\prime}=a_{i} / b_{i}>0$ in the first case and $\gamma_{i}=0, a_{j}=\exp \left(y_{j}\right)$ in the second. This may involve the cancellation of factors of $x_{i}$ which appear on both sides of the equation and hence the loss of some solutions contained entirely in the boundary. In view of this we will define binomial structures on sets which are the closure of their intersection with the interior as in (9.4) below.

With this motivation in mind, on any manifold consider the set of functions

$$
\begin{gathered}
\mathcal{G}(X)=\bigcup_{\gamma: \mathcal{M}_{1}(X) \rightarrow \mathbb{Z}} \mathcal{G}_{\gamma}(X), \text { where } \\
\mathcal{G}_{\gamma}(X)=\left\{u \in C^{\infty}(X \backslash \partial X) ; u=a \rho^{\gamma}, 0<a \in C^{\infty}(X)\right\} ;
\end{gathered}
$$

here $\rho=\left(\rho_{H}\right)_{H \in \mathcal{M}_{1}(X)}$ is a collection of global boundary defining functions. Observe that $\gamma: \mathcal{M}_{1}(X) \longrightarrow \mathbb{Z}$ induces a functional ${ }^{\mathrm{b}} \gamma:{ }^{\mathrm{b}} N G \longrightarrow \mathbb{C}$ for any $G \in \mathcal{M}(X)$ by setting

$$
{ }^{\mathrm{b}} \gamma=\sum_{\mathcal{M}_{1}(X) \ni H \supseteq G} \gamma(H) \frac{d \rho_{H}}{\rho_{H}}
$$


The $\mathcal{G}_{\gamma}(X)$ are independent of the defining functions used in (9.2) and pull back under any interior b-map $f: Z \longrightarrow Y$ giving an inclusion $f^{*} \mathcal{G}(Y) \subset \mathcal{G}(X)$. Clearly $\mathcal{G}(X)$ is an abelian group under pointwise multiplication, and $\mathcal{G}_{\gamma_{1}}(X) \cdot \mathcal{G}_{\gamma_{2}}(X) \subset$ $\mathcal{G}_{\gamma_{1}+\gamma_{2}}(X)$.

A function $f \in \mathcal{G}(X)$ can be extended by continuity to points in $\partial X$ at which it has finite limits, but the logarithmic differential, $d f / f$, extends by continuity to a smooth global section of ${ }^{\mathrm{b}} T^{*} X$ since near the boundary it reduces to

$$
\frac{d f}{f}=d \log a+\sum_{H} \gamma(H) \frac{d \rho_{H}}{\rho_{H}} .
$$

Definition 9.1. A local binomial structure on a closed subset $D \subset X$ near a point $p \in D$ consists of a coordinate neighborhood $U \ni p$ and functions $f_{i} \in \mathcal{G}_{\gamma_{i}}(U)$, $i=1, \ldots, d$, which have independent logarithmic differentials $d f_{i} / f_{i} \in{ }^{\mathrm{b}} T_{q}^{*} U$ at each $q \in D \cap U$, and which define $D$ locally in the sense that

$$
D \cap U=\cos _{U}\left\{q \in U \backslash \partial U ; f_{i}(q)=1, i=1, \ldots, d\right\} .
$$

The codimension of $D$ at $p$ is $d$.

An exponent vector $\gamma \in \mathcal{M}_{1}(X) \longrightarrow \mathbb{Z}$ is said to be non-negative (resp. nonpositive) if $\gamma(H) \geq 0$ (resp. $\leq 0$ ) for all $H \in \mathcal{M}_{1}(X)$, and $\gamma$ is indefinite if it is non-zero, and neither non-negative nor non-positive, so an indefinite $\gamma$ must have at least one positive and at least one negative coefficient. More locally, $\gamma$ is non-negative with respect to $F \in \mathcal{M}(X)$ if $\gamma(H) \geq 0$ for all $H \in \mathcal{M}_{1}(X)$ such that $F \subseteq H$, and non-positivity and indefiniteness with respect to $F$ are defined similarly.

For a local binomial structure $U$ on $D \subset X$, the boundary faces of $X \cap U$ which are met by $D$ can be seen by examining the indefiniteness of the exponent vectors.

Lemma 9.2. If $U, f_{i} \in \mathcal{G}_{\gamma_{i}}(U)$, is a local binomial structure on $D$ near $p$ and $U$ only meets boundary hypersurfaces which pass through $p$, then every exponent vector $\gamma_{i}$ is either zero or indefinite. Similarly, if $D$ meets the interior of $F \in \mathcal{M}(U)$, then each $\gamma_{i}$ is either zero or indefinite with respect to $F$.

Proof. By assumption all the local boundary defining functions vanish at $p$, so if $\gamma_{i}$ is non-zero and has all entries of a fixed sign, then $a_{i} x^{\gamma_{i}}$ cannot be equal to 1 near $p$. The same argument applies to other boundary points of $D$ where fewer of the boundary defining functions vanish.

Next we establish a local normal form for local binomial structures.

Lemma 9.3. If $U, f_{i} \in \mathcal{G}_{\delta_{i}}(U)$, is a local binomial structure on $D$, then near any boundary point $q \in D$ in $U$ there are local coordinates $(x, y)$ in a (possibly smaller) neighborhood $U^{\prime}$ of $q$ in terms of which

$$
D \cap U^{\prime}=\left\{x^{\gamma_{i}}=1, i=1, \ldots, d^{\prime}, y_{j}=0, j=d^{\prime}+1, \ldots, d\right\},
$$

where the $\gamma_{i}$ are linearly independent and indefinite vectors with respect to the maximal codimension boundary face through $q$.

Proof. By definition of local binomial structure, $D \cap U$ is the closure of its intersection with the interior, so there are interior points of $D$ near $q$. Let $F$ be the boundary face of maximal codimension containing $q$; so $q$ lies in the interior of $F$. The b-cotangent space ${ }^{\mathrm{b}} T_{q}^{*} U$, of which the $d \log f_{i}$ are sections, has a 'smooth 
subspace' ${ }^{\mathrm{b}} N_{q}^{\perp} F$ consisting of the differentials of smooth functions. It is the image in ${ }^{\mathrm{b}} T_{q}^{*} U$ of the natural map $T_{q}^{*} U \longrightarrow{ }^{\mathrm{b}} T_{q}^{*} U$ and is the annihilator of the b-normal space ${ }^{\mathrm{b}} N_{q} F$.

The assumed independence of the logarithmic differentials $d f_{i} / f_{i}$ in $U$ ensures that they span a linear space of dimension $d$ at $q$,

$$
A_{q}(D):=\operatorname{span}\left\{d f_{i} / f_{i}\right\} \subset{ }^{\mathrm{b}} T_{q}^{*} U .
$$

Within this space consider the intersection

$$
A_{q}^{\mathrm{sm}}(D)=A_{q}(D) \cap{ }^{\mathrm{b}} N_{q}^{\perp} F
$$

with the smooth subspace and set $d-d^{\prime}=\operatorname{dim}\left(A_{q}^{\mathrm{sm}}(D)\right)$. In terms of local coordinates $(x, y)$ at $q$, the functions $f_{i}=a_{i} x^{\delta_{i}^{\prime}}$, where the $\delta_{i}^{\prime}$ are the restrictions of the $\delta_{i}$ to the boundary hypersurfaces through $q$ and ${ }^{\mathrm{b}} N_{q}^{\perp} F$ is the span of the $d y_{k}$. Thus $A_{q}^{\mathrm{sm}}(D)$ is spanned by those linear combinations of logarithmic differentials

$$
\sum_{i} t_{i} d f_{i} / f_{i}=\sum_{i, j} t_{i} \delta_{i j}^{\prime} d x_{j} / x_{j}+\sum_{i} t_{i} d \log a_{i}
$$

for which $\sum_{i} t_{i} \delta_{i}^{\prime} \equiv 0$.

Thus, after some renumbering, an independent set of the $\delta_{i}^{\prime}$ can be chosen and renamed $\gamma_{i}, i=1, \ldots, d^{\prime}$. The remaining $f_{j}, j=d^{\prime}+1, \ldots, d$, can be replaced by products $f_{j}^{\prime}=\prod_{i} f_{i}^{t_{i}}$ corresponding to non-trivial independent relations $\sum_{i} t_{i} \delta_{i}^{\prime}=$ 0 . Then the set $\left\{d f_{i} / f_{i}, d f_{j}^{\prime} / f_{j}^{\prime}\right\}$ is independent and spans $A_{q}(D)$ and $D$ is given locally by the equations $f_{i}=f_{j}^{\prime}=1$, but the $f_{j}^{\prime}=a_{j}^{\prime} x^{0}$ are smooth and positive.

The functions $\log a_{j}^{\prime}, j=d^{\prime}+1, \ldots, d$, can then be introduced in place of some of the tangential variables $y_{j}$, and their differentials span $A_{q}^{\mathrm{sm}}(D)$. Since the $\gamma_{i}$ are now linearly independent, after changing the boundary variables from $x_{k}$ to $g_{k} x_{k}$ with $g_{k}>0$ and renumbering, $a_{i} x^{\gamma_{i}}$ is reduced to the desired form $x^{\gamma_{i}}$ giving (9.5).

Definition 9.4. A connected, closed subset $D \subset X$ of a manifold with corners is an interior binomial subvariety if it has a covering by local binomial structures.

The codimension of $D$ is well defined as the local codimension by connectivity, and $D \cap(X \backslash \partial X)$ is a smooth manifold of dimension $\operatorname{dim}(D)=\operatorname{dim}(X)-\operatorname{codim}(D)$.

It follows from the proof of Lemma 9.3 that the 'b-conormal spaces' $A_{q}(D) \subset$ ${ }^{\mathrm{b}} T_{q}^{*} X$ in (9.6) are well defined at each point $q \in D$ and independent of the local binomial structure used - for points in the interior $A_{q}(D)$ is just the ordinary conormal space to the smooth manifold $D \cap X \backslash \partial X$, and its extension by continuity to $D \cap \partial X$ is unique. So we may proceed as for a smooth submanifold of a manifold and set

$$
\begin{gathered}
{ }^{\mathrm{b}} T_{q} D=\left(A_{q}(D) \subset{ }^{\mathrm{b}} T_{q}^{*} X\right)^{\perp} \subset{ }^{\mathrm{b}} T_{q} X, \\
{ }^{\mathrm{b}} N_{q} D_{G}={ }^{\mathrm{b}} T_{q} D \cap{ }^{\mathrm{b}} N_{q} G, \quad p \in D \cap G, G \in \mathcal{M}(X) .
\end{gathered}
$$

The b-tangent bundle ${ }^{\mathrm{b}} T D \longrightarrow D$ is actually independent of the 'binomial embedding' of $D$ in $X$ once this is understood correctly, but here we persist with the extrinsic discussion. Observe that each ${ }^{\mathrm{b}} N_{q} D_{G}$ is just the nullspace of the maps ${ }^{\mathrm{b}} \gamma_{i}$ as in (9.3) for the $\gamma_{i}$ occuring in any local binomial structure for $D$ near $p$. In particular, it is invariant with respect to replacing the $\gamma_{i}$ by linear combinations with the same span. 
From the local normal form (9.5) it follows that the intersection of $D$ with a boundary face through $p$ is again a binomial subvariety, as we now show. By way of motivation, notice the behavior of the b-tangent spaces on passage to a boundary face of $X$ : for $p \in G \in \mathcal{M}(X),{ }^{\mathrm{b}} T_{p} G={ }^{\mathrm{b}} T_{p} X /{ }^{\mathrm{b}} N_{p} G$.

Lemma 9.5. Suppose $D \subset X$ is an interior binomial variety with codimension $d$. Then for any $G \in \mathcal{M}(X)$, such that $D \cap(G \backslash \partial G) \neq \emptyset$, a component $G^{\prime}$ of the closure $D_{G}:=\operatorname{clos}(D \cap G \backslash \partial G)$ is an interior binomial subvariety of $G$ with codimension $d-\operatorname{dim}\left({ }^{\mathrm{b}} N_{p} D_{G}\right)$, for any $p \in G^{\prime}$ and

$$
{ }^{\mathrm{b}} T_{p} D_{G}={ }^{\mathrm{b}} T_{p} D /{ }^{\mathrm{b}} N_{p} D_{G} .
$$

It may well happen that $D \cap(G \backslash \partial G)=\emptyset$ but $D \cap G \neq \emptyset$, in which case we do not regard $D \cap G$ as an interior binomial subvariety of $G$ since it lies only in the boundary.

Proof. Let $p$ be a limit point of $G^{\prime} \cap G \backslash \partial G$, and consider the local form for $D$ near $p$ given on a neighborhood $U^{\prime}$ by (9.5); we can assume without loss of generality that $U^{\prime}$ does not meet any other component of $D \cap G$. Thus the $x$ 's are local defining functions for the boundary face $F \subseteq G$ of maximal codimension through $p$. Divide them as $x=\left(x^{\prime}, x^{\prime \prime}\right)$, where the $x^{\prime \prime}$ define $G$ locally.

Following the proof of Lemma 9.3 above, a maximal subset of the $\gamma_{i}$ may be chosen so that their restrictions to the boundary faces containing $G$ are independent, i.e. the projections $\gamma_{i}=\left(\gamma_{i}^{\prime}, \gamma_{i}^{\prime \prime}\right) \longmapsto \gamma_{i}^{\prime \prime}$ onto the subspace corresponding to the $x^{\prime \prime}$ are independent. The remaining $\gamma_{j}$ may be replaced by independent linear combinations $\delta_{j}=\sum_{i} t_{i} \gamma_{i}$ which are non-zero only with respect to the boundary hypersurfaces which do not contain $G$, i.e. their projections $\delta_{j}=\left(\delta_{j}^{\prime}, \delta_{j}^{\prime \prime}\right) \longmapsto \delta_{j}^{\prime \prime}$ vanish. The defining conditions (9.5) may therefore be rewritten, after renumbering,

$$
\begin{gathered}
\left(x^{\prime}\right)^{\gamma_{i}^{\prime}}\left(x^{\prime \prime}\right)^{\gamma_{i}^{\prime \prime}=1, i}=1, \ldots, k,\left(x^{\prime}\right)^{\delta_{i}^{\prime}}=1, i=k+1, \ldots, d^{\prime}, \\
y_{j}=0, j=d^{\prime}+1, \ldots, d,
\end{gathered}
$$

where the $\gamma_{i}^{\prime \prime}$ and $\delta_{i}^{\prime}$ are separately independent, and the $\gamma_{i}^{\prime \prime} d x_{i}^{\prime \prime} / x_{i}^{\prime \prime}$ span ${ }^{\mathrm{b}} N_{p} D_{G}$.

Then

$$
G^{\prime} \cap U^{\prime}=\left\{\left(x^{\prime}\right)^{\gamma_{i}^{\prime}}=1, i=k+1, \ldots, d^{\prime}, y_{j}=0, j=d^{\prime}+1, \ldots, d\right\}
$$

is a local binomial structure on $G^{\prime}$. Certainly the right side of (9.10) is included in the left. To see the converse, observe that, by the assumption that $D$ meets the interior of $G$ near $p$, the system (9.9) must have a sequence of solutions with $x^{\prime \prime} \rightarrow 0$ and all entries of $x^{\prime}$ positive but small.

Writing the first set of equations as the linear system $\gamma_{i}^{\prime \prime} \cdot \log x^{\prime \prime}=c_{i}$ for the vector $\log x^{\prime \prime}$, with entries the logarithms of the $x^{\prime \prime}$, we see that this system must, for each $N \in \mathbb{R}$, have a solution with all entries less than $N$ with $c_{i}$ bounded. Since $\gamma_{i}^{\prime \prime}$ are linearly independent, it follows that the same is true for any $c_{i}$. Thus in fact any solution of the equations in (9.10) corresponds to a point in $G^{\prime}$.

In light of this result, we define the boundary faces of $D$ to be the components $G^{\prime}$ of the non-empty $D_{G}=\operatorname{clos}(D \cap G \backslash \partial G)$. The linear space ${ }^{\mathrm{b}} N_{p} G^{\prime}:={ }^{\mathrm{b}} N_{p} D_{G}=$ Null $\left\{{ }^{\mathrm{b}} \gamma_{i}\right\} \subset{ }^{\mathrm{b}} N_{p} G$ as in (9.7) can then be identified as the 'b-normal space to $G^{\prime}$ as a boundary face of $D^{\prime}$, consistent with the smooth case. The codimension of a boundary face $G^{\prime} \subset D_{G}$ with respect to $D$ is given by $\operatorname{dim}\left({ }^{\mathrm{b}} N G^{\prime}\right)$ as expected, 
and we let $\mathcal{M}_{k}(D)$ be the boundary faces of codimension $k$ in this sense, equipping $\mathcal{M}(D)=\bigcup_{k} \mathcal{M}_{k}(D)$ with the order of reverse inclusion.

The intersection of the rational subspaces ${ }^{\mathrm{b}} N G^{\prime}, G^{\prime} \in \mathcal{M}(D)$, with the monoids $\sigma_{G}, G \in \mathcal{M}(X)$, gives the 'basic monoidal complex' of $D$.

Proposition 9.6. If $D \subset X$ is an interior binomial subvariety the monoids

$$
\sigma_{G^{\prime}}={ }^{\mathrm{b}} N G^{\prime} \cap \sigma_{G}, G^{\prime} \in \mathcal{M}(D),
$$

where $G^{\prime}$ is a component of $\cos (D \cap G \backslash \partial G)$, form a (not necessarily smooth) complex $\mathcal{P}_{D}$ over $(\mathcal{M}(D), \leq)$ and there is a natural, injective morphism of complexes

$$
i_{\natural}: \mathcal{P}_{D} \longrightarrow \mathcal{P}_{X}
$$

over $i_{\#}: \mathcal{M}(D) \longrightarrow \mathcal{M}(X)$, where $i_{\#}\left(G^{\prime}\right)=G$ such that $G^{\prime} \subset D_{G}$ as above.

Proof. The monoids $\sigma_{G^{\prime}}$ are clearly well defined and toric; it suffices to verify that they form a complex. If $F^{\prime} \subseteq G^{\prime} \in \mathcal{M}(D)$ and $p \in F^{\prime}$, then $F \subseteq G \in \mathcal{M}(X)$. Since ${ }^{\mathrm{b}} N_{p} G^{\prime}={ }^{\mathrm{b}} T_{p} D \cap{ }^{\mathrm{b}} N_{p} G$ and similarly for ${ }^{\mathrm{b}} N_{p} F^{\prime}$, and since ${ }^{\mathrm{b}} N_{p} G \subseteq{ }^{\mathrm{b}} N_{p} F$, it follows that ${ }^{\mathrm{b}} N_{p} G^{\prime} \subseteq{ }^{\mathrm{b}} N_{p} F^{\prime}$, giving an inclusion $\sigma_{G^{\prime}} \subset \sigma_{F^{\prime}}$, which must be an isomorphism onto a face since it is the intersection of $\sigma_{F}$ with a subspace. Thus

$$
\mathcal{P}_{D}=\left\{\sigma_{G} ; G \in \mathcal{M}(D)\right\}
$$

is a (complete, reduced) monoidal complex over $\mathcal{M}(D)$, and the inclusions $\sigma_{G} \subset$ $\sigma_{i_{\#}(G)}$ produce a necessarily injective morphism $i: \mathcal{P}_{D} \longrightarrow \mathcal{P}_{X}$.

A very special instance of an interior binomial subvariety is a 'product-' or $p$ submanifold (see Mel for background information). This is a smooth submanifold $D \subset X$ which meets all boundary faces of $X$ transversally and which is covered by coordinate neighborhoods $(U,(x, y))$ in $X$ such that

$$
D \cap U=\left\{y_{j}=0 ; j=1, \ldots, \operatorname{codim}(Y)\right\} .
$$

For a p-submanifold, it is evident that ${ }^{\mathrm{b}} N_{p} G^{\prime} \equiv{ }^{\mathrm{b}} N_{p} G$ whenever $G^{\prime} \in \mathcal{M}(D)$ is a component of $D_{G}, G \in \mathcal{M}(X)$, and so the morphism $i_{\natural}: \mathcal{P}_{D} \longrightarrow \mathcal{P}_{X}$ consists of monoid isomorphisms $\sigma_{G^{\prime}} \stackrel{\cong}{\longrightarrow} \sigma_{G}$. In other words $i_{\natural}$ is a local isomorphism, though it need not be a global one, since the $D_{G}$ may consist of multiple components, and therefore $i_{\#}: \mathcal{M}(D) \longrightarrow \mathcal{M}(X)$ need not be injective.

In fact this global issue of the failure of $i_{\#}: \mathcal{M}(D) \longrightarrow \mathcal{M}(X)$ to be injective arises as a technical obstruction to the resolution of a general interior binomial variety $D \subset X$ by the generalized blow-up of $X$ in the next section. Indeed, if there are multiple components $G_{i}^{\prime} \subset D_{G}$, there may be no way to refine $\sigma_{G} \in \mathcal{P}_{X}$ in a way which appropriately resolves the images $i_{\natural}\left(\sigma_{G_{i}^{\prime}}\right)$. Fortunately, one can always pass to a 'collar neighborhood' $X^{\prime} \supset D$ in $X$ for which this obstruction does not arise. The following lemma guarantees the existence of such a neighborhood; the proof follows directly from the local normal form (9.5).

Lemma 9.7. If $D \subset X$ is an interior binomial subvariety, then there exists an open submanifold $X^{\prime} \subset X$ containing $D$ such that $i_{\#}: \mathcal{M}(D) \longrightarrow \mathcal{M}\left(X^{\prime}\right)$ is injective so that for each $G \in \mathcal{M}\left(X^{\prime}\right)$ there is at most one connected component of $D_{G}$.

Finally, observe that other concepts can be extended from the 'smooth' case of manifolds to binomial subvarieties. 
Definition 9.8. If $D \subset X$ is a binomial subvariety and $Y$ a manifold with corners, then a map $f: Y \longrightarrow D$ is a b-map if it is a b-map in the smooth sense, i.e. as a map $f: Y \longrightarrow X$ with $f(Y) \subset D$.

For such a b-map, the range of the b-differential ${ }^{\mathrm{b}} f_{*}$ will lie in ${ }^{\mathrm{b}} T D$, and if $G^{\prime} \in \mathcal{M}(D)$ is the highest codimension face such that $f(F) \subset G^{\prime}$, it follows that

$$
{ }^{\mathrm{b}} f_{*}:{ }^{\mathrm{b}} N_{p} F \longrightarrow{ }^{\mathrm{b}} N_{f(p)} G^{\prime} .
$$

Just as in Definition 6.1 there is an induced map

$$
f_{\natural}: \mathcal{P}_{Y} \longrightarrow \mathcal{P}_{D}
$$

of monoidal complexes.

\section{Resolution of BinOMial SUbVARIETIES}

In this section we show that a carefully chosen smooth refinement of the monoidal complex of the ambient manifold $X$ which also refines the monoidal complex of an interior binomial subvariety $D \subset X$ leads to a blow-up under which $D$ lifts to a p-submanifold; in particular, this resolves $D$. The resolution of $D$ so obtained depends essentially only on the choice of the smooth refinement of the monoidal complex $\mathcal{P}_{D}$.

The notion of a resolution is analogous to that of a generalized blow-up defined earlier.

Definition 10.1. If $D \subset X$ is an interior binomial subvariety, then a manifold $Y$ with a b-map $f: Y \longrightarrow X$ with $f(Y) \subset D$ is a resolution of $D$ if ${ }^{\mathrm{b}} f_{*}:{ }^{\mathrm{b}} T_{p} Y \longrightarrow$ ${ }^{\mathrm{b}} T_{f(p)} D$ is a bijection for all $p \in Y$ and $f: Y \backslash \partial Y \longrightarrow D \backslash \partial D$ is a diffeomorphism.

Proposition 10.2. If $\beta: X_{1} \longrightarrow X$ is a generalized blow-down map between manifolds and $D \subset X$ is an interior binomial subvariety, then the lift (or proper transform)

$$
\beta^{\#}(D)=\operatorname{clos}_{X_{1}}\left(\beta^{-1}(D \backslash \partial X)\right)
$$

is an interior binomial subvariety of $X_{1}$.

Proof. Certainly $\beta^{\#}(D) \subset X_{1}$ is a closed subset. It is a smooth embedded submanifold in the interior, so it suffices to show that it has a local binomial structure at each boundary point. If $p \in \partial X_{1} \cap \beta^{\#}(D)$, then by definition, $\beta(p) \in \partial X \cap D$ and $D$ has a local binomial structure given by $f_{i} \in \mathcal{G}(X)$. The pull-backs $f^{*} f_{i} \in \mathcal{G}\left(X_{1}\right)$ define $\beta^{\#}(D)$ locally, and their logarithmic differentials are independent since the b-differential of $\beta$ is an isomorphism at each point.

Let $\mathcal{R} \longrightarrow \mathcal{P}_{X}$ be a smooth refinement. If $D \subset X$ is an interior binomial subvariety, then $\mathcal{R}$ is compatible with $\mathcal{P}_{D}$ if there is a subcomplex $\mathcal{R}_{D} \subset \mathcal{R}$ such that $\mathcal{R}_{D} \longrightarrow \mathcal{P}_{X}$ factors through $\mathcal{P}_{D}$ giving a commutative diagram

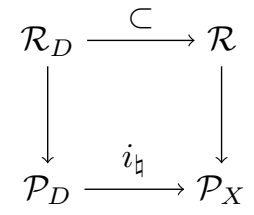

where the vertical arrows are smooth refinements and the top is the inclusion of a subcomplex. 
Proposition 10.3. If $\mathcal{R} \longrightarrow \mathcal{P}_{X}$ is a smooth refinement which is compatible with an interior binomial subvariety $D$ in the sense of (10.2), then $D$ lifts to a p-submanifold $\widetilde{D}=\beta^{\#}(D)$ of the generalized blow-up

$$
\beta:[X ; \mathcal{R}] \longrightarrow X
$$

In particular, $\beta: \widetilde{D} \longrightarrow D$ is a resolution of $D$ and $\beta_{\natural}: \mathcal{P}_{\widetilde{D}} \longrightarrow \mathcal{P}_{D}$ factors through an isomorphism $\mathcal{P}_{\widetilde{D}} \cong \mathcal{R}_{D}$ of monoidal complexes.

Proof. Consider an arbitrary $p^{\prime} \in \widetilde{D}=\beta^{\#}(D)$, and let $p=\beta\left(p^{\prime}\right) \in D$. There is a unique face $G^{\prime} \in \mathcal{M}(D)$ such that $p$ lies in the interior of $G^{\prime}$, and we set $G=i_{\#}\left(G^{\prime}\right) \in \mathcal{M}(X)$. Since $i_{\natural}: \mathcal{P}_{D} \rightarrow \mathcal{P}_{X}$ in (9.12) and $\beta_{\natural}: \mathcal{R} \longrightarrow \mathcal{P}_{X}$ are both injective, we identify monoids $\sigma_{G^{\prime}}$ and $\tau \in \mathcal{R}(G)$ with their respective images in $\sigma_{G}$.

The point $p^{\prime} \in \widetilde{D} \subset[X ; \mathcal{R}]$ lies in some coordinate chart $U_{\tau} \subset \mathbb{R}_{+}^{k} \times(0, \infty)^{n-k}$ with coordinates $(t, y)=\left(t_{1}, \ldots, t_{k}, y_{1}, \ldots, y_{n-k}\right)$, for some $\tau \in \mathcal{R}(G)$, and we can assume that the coordinates $y$ are pulled back identically from those on $X$ in which $D$ has the local normal form (9.5). Thus the equations $\left\{y_{j}=0 ; j=d^{\prime}+1, \ldots, d\right\}$ are the same in $U_{\tau}$. We consider the lift of the other equations, $x^{\gamma_{i}}=1$ under $\beta$. On $U_{\tau}, \beta$ has the form $\beta: t \longrightarrow t^{\mu}=x$, and we obtain

$$
\beta^{*}\left(x^{\gamma_{i}}\right)=\left(t^{\mu}\right)^{\gamma_{i}}=t^{\beta_{i}}=1,
$$

where $\beta_{i}=\mu \gamma_{i}$. Thus near $p^{\prime}, \widetilde{D} \subset[X ; \mathcal{R}]$ has the local binomial structure

$$
\widetilde{D}=\left\{t^{\beta_{i}}=1, y_{j}=0\right\} .
$$

We will show that the $\beta_{i}$ are each non-negative or non-positive.

Indeed, the compatibility assumption implies that, for all $\tau \in \mathcal{R}(G)$, either $\tau \in \mathcal{R}_{D}\left(G^{\prime}\right)$ and hence $\tau \subset \sigma_{G^{\prime}}$, or $\tau \in \mathcal{R} \backslash \mathcal{R}_{D}$; in either case the intersection $\tau \cap \sigma_{G^{\prime}}$ must be a face of $\tau$. Since $\sigma_{G^{\prime}}=\sigma_{G} \cap \operatorname{Null}\left\{{ }^{\mathrm{b}} \gamma_{i}\right\}$, it follows that

$$
\left\langle\gamma_{i} d x / x, \tau\right\rangle \geq 0 \text { or }\left\langle\gamma_{i} d x / x, \tau\right\rangle \leq 0, \quad \text { for each } \gamma_{i} \text {. }
$$

In other words, each vector $\gamma_{i} d x / x$ is either non-positive or non-negative with respect to $\tau \in \mathcal{R}(G)$. Let us assume non-negative; the other case is similar.

From this it follows that no $\beta_{i}$ is indefinite, since for any $a \in \mathbb{R}^{k}, a_{i}>0$,

$$
\left\langle\beta_{i}, a\right\rangle=\left\langle\beta_{i} d t / t, a t \partial_{t}\right\rangle=\left\langle\mu \gamma_{i} d x / x, a t \partial_{t}\right\rangle=\left\langle\gamma_{i} d x / x, \mu^{\mathrm{T}}\left(a t \partial_{t}\right)\right\rangle \geq 0
$$

as $\mu^{\mathrm{T}}\left(a t \partial_{t}\right) \in \tau$. In light of Lemma 9.2, $\widetilde{D}$ only meets boundary faces with respect to which the $\beta_{i}$ are zero; hence $\widetilde{D}$ has a covering by binomial structures such that

$$
\widetilde{D}=\left\{y_{j}=0 ; j=1, \ldots, d\right\}
$$

and is therefore a p-submanifold.

Since $\widetilde{D}$ is a p-submanifold, for any $\widetilde{G} \in \mathcal{M}(\widetilde{D}), \sigma_{\widetilde{G}} \cong \tau$ for some $\tau \in \mathcal{R} \cong \mathcal{P}_{[X ; \mathcal{R}]}$. Finally, it follows from the fact that $\widetilde{D}$ is the lift of $D$ that $\sigma_{\widetilde{G}}=\tau$ is actually in $\mathcal{R}_{D}$; hence $\mathcal{P}_{\widetilde{D}} \longrightarrow \mathcal{R}_{D}$ is a local isomorphism onto its image and $\beta: \widetilde{D} \longrightarrow D$ is a resolution since it is a diffeomorphism on interiors.

The resolution of binomial ideals in polynomial and power series rings by toric methods are well known; see Tei] for a good overview. The previous proposition can be seen as an extension of this theory to the interior binomial subvarieties we have been discussing. 
Next we show that we can obtain a unique resolution of $D$ realizing any smooth refinement $\mathcal{R}_{D} \longrightarrow \mathcal{P}_{D}$; in particular, the resolution so obtained is essentially independent of the ambient manifold $X$.

Theorem 10.4 (Resolution of binomial varieties). If $D \subset X$ is an interior binomial subvariety, then for every smooth refinement $\mathcal{R}_{D} \longrightarrow \mathcal{P}_{D}$ there exists a resolution

$$
\beta:\left[D ; \mathcal{R}_{D}\right] \longrightarrow D
$$

which realizes the refinement in the sense that

$$
\beta_{\natural}: \mathcal{P}_{\left[D ; \mathcal{R}_{D}\right]} \cong \mathcal{R}_{D} \longrightarrow \mathcal{P}_{D},
$$

and $\left[D ; \mathcal{R}_{D}\right]$ is unique up to diffeomorphism.

If $f: Y \longrightarrow D$ is a b-map from a smooth manifold, and if $f_{\mathfrak{\natural}}: \mathcal{P}_{Y} \longrightarrow \mathcal{P}_{D}$ factors through $\mathcal{R}_{D}$, then $f$ factors through a unique b-map $\tilde{f}: Y \longrightarrow\left[D ; \mathcal{R}_{D}\right]$.

The notation is meant to suggest that this is in some sense the generalized blow-up in the category of (differentiable) 'binomial varieties' where the objects are treated intrinsically. Indeed, we believe that such a category exists and that generalized blow-up extends to include arbitrary refinements, not necessarily smooth.

Proof. Assume that $i_{\#}: \mathcal{M}(D) \longrightarrow \mathcal{M}(X)$ is injective, passing if necessary to a collar neighborhood $X^{\prime} \subset D$ as in Lemma 9.7. By Proposition [10.3 it suffices to show that, given $\mathcal{R}_{D} \longrightarrow \mathcal{P}_{D}$, there exists a refinement $\mathcal{R}_{X} \longrightarrow \mathcal{P}_{X}$ extending $\mathcal{R}_{D}$ (that is, containing $\mathcal{R}_{D}$ as a subcomplex), and that the resulting resolution $\beta^{\#}(D) \subset\left[X ; \mathcal{R}_{X}\right]$ is well defined, independent of the choice of such an extension.

For the first step the planar refinement of Proposition 4.10, $\mathcal{S}\left(\mathcal{P}_{X}, \mathcal{P}_{D}\right) \longrightarrow \mathcal{P}_{X}$, is a (not necessarily smooth) refinement containing $\mathcal{P}_{D}$ as a subcomplex. Then by Lemma 5.6 the refinement $\mathcal{R}_{D} \longrightarrow \mathcal{P}_{D}$ can be extended to a smooth refinement $\mathcal{R}_{X} \longrightarrow \mathcal{S}\left(\mathcal{P}_{X}, \mathcal{P}_{D}\right)$, and the composition $\mathcal{R}_{X} \rightarrow \mathcal{S}\left(\mathcal{P}_{X}, \mathcal{P}_{D}\right) \rightarrow \mathcal{P}_{X}$ is therefore an extension of $\mathcal{R}_{D}$.

Next suppose $\mathcal{R}_{1}$ and $\mathcal{R}_{2}$ are smooth refinements of $\mathcal{P}_{X}$ extending $\mathcal{R}_{D}$, and set

$$
\widetilde{D}_{i}:=\beta_{i}^{\#}(D) \subset\left[X ; \mathcal{R}_{i}\right], \quad i=1,2 .
$$

The blow-down $\beta_{1}: \widetilde{D}_{1} \longrightarrow D$, considered as a map to $X$, lifts by Theorem 6.3 to a b-map to $\left[X ; \mathcal{R}_{2}\right]$ whose range lies in $\widetilde{D}_{2}$, and vice versa. Thus we obtain b-maps

$$
\widetilde{D}_{1} \leftrightarrow \widetilde{D}_{2}
$$

which are generalized blow-down maps and in fact diffeomorphisms by Proposition 8.6 since $\mathcal{P}_{\widetilde{D}_{1}} \cong \mathcal{R}_{D} \cong \mathcal{P}_{\widetilde{D}_{2}}$.

Thus the lift $\widetilde{D} \subset\left[X ; \mathcal{R}_{X}\right]$ is independent of the extension $\mathcal{R}_{X}$ of $\mathcal{R}_{D}$ up to diffeomorphism, and we define $\left[D ; \mathcal{R}_{D}\right]=\widetilde{D}$ to be any such lift.

Recall that a b-map $f: Y \longrightarrow D$ induces a monoidal complex morphism $f_{\natural}$ :

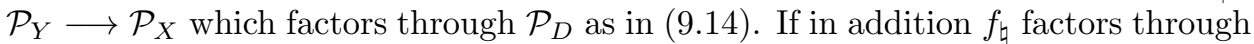
$\mathcal{R}_{D} \longrightarrow \mathcal{P}_{D}$, then it follows that $f_{\natural}: \mathcal{P}_{Y} \longrightarrow \mathcal{P}_{X}$ factors through any extension $\mathcal{R}_{X}$, and $f$ admits a unique lift $f^{\prime}: Y \longrightarrow\left[X ; \mathcal{R}_{X}\right]$ by Theorem 6.3 . The image of $Y \backslash \partial Y$ under $f^{\prime}$ lies in $\widetilde{D} \backslash \partial\left[X ; \mathcal{R}_{X}\right]$, and therefore by continuity $f^{\prime}(Y) \subset \widetilde{D}$, so $f^{\prime}: Y \longrightarrow \widetilde{D}$ is a b-map. Since the resolutions coming from different extensions are diffeomorphic, it follows from the naturality of the lifted b-maps that

$$
f^{\prime}: Y \longrightarrow\left[D ; \mathcal{R}_{D}\right]
$$

is well defined, independent of the choice of extension. 
Observe that if $\mathcal{P}_{D}$ is already smooth, then the above suggests that $D$ is in some sense already a smooth manifold, though it may not be nicely embedded in $X$. Indeed, it follows from the proof of Theorem 10.4 that there exists a refinement $\mathcal{R}_{X} \longrightarrow \mathcal{P}_{X}$ which is trivial on $\mathcal{P}_{D}$, and this gives a 'minimal' resolution of $D$ which is universal in this case.

Theorem 10.5. If $D \subset X$ is an interior binomial variety and $\mathcal{P}_{D}$ is smooth, then there exists a universal resolution $\left[D ; \mathcal{P}_{D}\right] \longrightarrow D$, with the property that any b-map $f: Y \longrightarrow D$ factors uniquely through $\left[D ; \mathcal{P}_{D}\right]$. In particular, any other resolution of $D$ is a generalized blow-up of this universal resolution.

Proof. From Theorem 10.4 there exists a unique resolution $\left[D ; \mathcal{P}_{D}\right]$ coming from the trivial refinement Id $: \mathcal{P}_{D} \longrightarrow \mathcal{P}_{D}$. Since any b-map $f: Y \longrightarrow D$ induces a morphism $f_{\natural}: \mathcal{P}_{Y} \longrightarrow \mathcal{P}_{D}$ which necessarily factors through this trivial refinement, $f$ necessarily factors through $\left[D ; \mathcal{P}_{D}\right]$. If $f$ itself is a resolution, it follows that the lift $f^{\prime}: Y \longrightarrow\left[D ; \mathcal{P}_{D}\right]$ is also diffeomorphic on the interiors, with bijective b-differential; in other words, it is a generalized blow-down map onto the manifold $\left[D ; \mathcal{P}_{D}\right]$, and hence a blow-up of this space by Theorem 8.7

In fact, though we shall not use this below, it is possible to show that in case $\mathcal{P}_{D}$ is smooth, the spaces $D$ and $\left[D ; \mathcal{P}_{D}\right]$ are actually homeomorphic. Indeed, it is straightforward to show that the map $\left[D ; \mathcal{P}_{D}\right]$ is a diffeomorphism on the interiors of boundary faces and hence globally bijective. Then since it is continuous and proper it has a continuous inverse. Thus one can regard Theorem 10.5 as giving a natural smooth structure on $D$ itself, though this is not generally equal to the restriction of the smooth structure on $X$, as illustrated by the example $D=\left\{x_{1}^{2}=x_{2}^{3}\right\} \subset \mathbb{R}_{+}^{2}$, whose universal resolution is the usual one: $\left[D ; \mathcal{P}_{D}\right]=\mathbb{R}_{+} \ni t \longmapsto\left(t^{3}, t^{2}\right) \subset \mathbb{R}_{+}^{2}$.

\section{FIBER PRODUCTS}

We next bring the theory of the last two sections to bear on the question of fiber products of manifolds with corners. Recall that in the category of manifolds without boundary, smooth fiber products do not generally exist. A sufficient condition in this context is transversality; namely, two smooth maps $f_{i}: X_{i} \longrightarrow Y, i=1,2$, are transversal if $\left(f_{1}\right)_{*}\left(T_{p_{1}} X_{1}\right)+\left(f_{2}\right)_{*}\left(T_{p_{2}} X_{2}\right)=T_{q} Y$ for all pairs $\left(p_{1}, p_{2}\right) \in X_{1} \times X_{2}$ such that $f_{1}\left(p_{1}\right)=f_{2}\left(p_{2}\right)=q$, and then

$$
X_{1} \times_{Y} X_{2}=\left\{\left(p_{1}, p_{2}\right) \in X_{1} \times X_{2} ; f_{1}\left(p_{1}\right)=f_{2}\left(p_{2}\right)\right\} \subset X_{1} \times X_{2}
$$

is a smooth manifold.

We show that the analogous condition of b-transversality in the category of manifolds with corners implies that $X_{1} \times_{Y} X_{2} \subset X_{1} \times X_{2}$ is a union of binomial subvarieties, each of which is interior to some product of faces. The theory we have developed then gives sufficient conditions for a fiber product (with the required universal properties) to exist in the category of manifolds with corners, and gives a coherent system of resolution by generalized blow-up even when these conditions are not fully satisfied.

Definition 11.1. Two interior b-maps $f_{i}: X_{i} \longrightarrow Y, i=1,2$, are $b$-transversal if for all points $p_{i} \in X_{i}$ such that $f_{1}\left(p_{1}\right)=f_{2}\left(p_{2}\right)=q \in Y$,

$$
{ }^{\mathrm{b}}\left(f_{1}\right)_{*}\left({ }^{\mathrm{b}} T_{p_{1}} X_{1}\right)+{ }^{\mathrm{b}}\left(f_{2}\right)_{*}\left({ }^{\mathrm{b}} T_{p_{2}} X_{2}\right)={ }^{\mathrm{b}} T_{q} Y .
$$


If the $f_{i}$ are not interior, then set $Y_{i}=\left(f_{i}\right)_{\#}\left(X_{i}\right) \in \mathcal{M}(Y)$ for $i=1,2$, so that $f_{i}: X_{i} \longrightarrow Y_{i}$ are interior b-maps. Consider the (possibly disconnected) manifold $Y_{1} \cap Y_{2}$. The preimages may be written

$$
f_{i}^{-1}\left(Y_{1} \cap Y_{2}\right)=\bigcup_{l} H_{i, l}
$$

$H_{i, l} \in \mathcal{M}\left(X_{i}\right)$ and $\left(f_{i}\right)_{\#}\left(H_{i, l}\right)$ is a component of $Y_{1} \cap Y_{2}$.

In particular, for each $H_{i, l}$ in (11.2), the induced b-map $f_{i}: H_{i, l} \longrightarrow Y_{1} \cap Y_{2}$ is interior.

Definition 11.2. Two general b-maps $f_{i}: X_{i} \longrightarrow Y, i=1,2$, are $b$-transversal if, in terms of the notation above, for all points $p_{i} \in H_{i, l_{i}}$ such that $f_{1}\left(p_{1}\right)=f_{2}\left(p_{2}\right)=$ $q \in Y$

$$
{ }^{\mathrm{b}}\left(f_{1}\right)_{*}\left({ }^{\mathrm{b}} T_{p_{1}} H_{1, l_{1}}\right)+{ }^{\mathrm{b}}\left(f_{2}\right)_{*}\left({ }^{\mathrm{b}} T_{p_{2}} H_{2, l_{2}}\right)={ }^{\mathrm{b}} T_{q}\left(Y_{1} \cap Y_{2}\right) .
$$

Thus in the general case the set-theoretic fiber product (11.1) of b-transversal maps is the union of the fiber products of interior b-maps.

Proposition 11.3 (Iterated transversality). If $f_{i}: X_{i} \longrightarrow Y, i=1,2$, are $b$ transversal, then for every pair of $F_{i} \in \mathcal{M}\left(X_{i}\right)$ such that $\left(f_{1}\right)_{\#}\left(F_{1}\right)=\left(f_{2}\right)_{\#}\left(F_{2}\right)=$ $F \in \mathcal{M}(Y)$, the induced b-maps

$$
\left(f_{i}\right)_{\mid F_{i}}: F_{i} \longrightarrow F
$$

are b-transversal.

Proof. The $F_{i}$ necessarily lie in $f_{i}^{-1}\left(Y_{1} \cap Y_{2}\right)$, and replacing the $X_{i}$ by maximal dimension faces $H_{i, l}$ of the latter such that $F_{i} \leq H_{i, l}$, we may assume that the $f_{i}$ are interior. Choose $p_{i} \in F_{i}$ such that $f_{1}\left(p_{1}\right)=f_{2}\left(p_{2}\right)=q$. Suppose $G_{i} \subseteq F_{i}$ are the maximal codimension boundary faces containing $p_{i}$ (in particular, $p_{i} \in G_{i} \backslash \partial G_{i}$ ), and set ${ }^{\mathrm{b}} N_{p_{i}}\left(G_{i} ; F_{i}\right)={ }^{\mathrm{b}} N_{p_{i}} G_{i} /{ }^{\mathrm{b}} N_{p_{i}} F_{i}$. These are the b-normal bundles to the $G_{i}$ in the manifolds $F_{i}$. The b-tangent spaces of the $G_{i}$ are the quotients ${ }^{\mathrm{b}} T_{p_{i}} G_{i}=$ ${ }^{\mathrm{b}} T_{p_{i}} X_{i} /{ }^{\mathrm{b}} N_{p_{i}} G_{i}$ with respect to the natural inclusions ${ }^{\mathrm{b}} N_{p_{i}} G_{i} \longleftrightarrow{ }^{\mathrm{b}} T_{p_{i}} X_{i}$, and since the $p_{i}$ lie in the interior of the $G_{i},{ }^{\mathrm{b}} T_{p_{i}} G_{i}=T_{p_{i}} G_{i}$.

Using a metric to replace the quotients by orthogonal decompositions,

$$
{ }^{\mathrm{b}} T_{p_{i}} X_{i}=T_{p_{i}} G_{i} \oplus{ }^{\mathrm{b}} N_{p_{i}}\left(G_{i} ; F_{i}\right) \oplus{ }^{\mathrm{b}} N_{p_{i}} F_{i} .
$$

The last two factors constitute ${ }^{\mathrm{b}} N_{p_{i}} G_{i}$, while the first two constitute ${ }^{\mathrm{b}} T_{p_{i}} F_{i}$. The b-differentials ${ }^{\mathrm{b}}\left(f_{i}\right)_{*}$ have the form

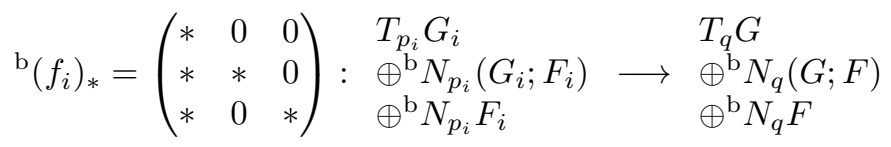

with respect to the quotients, where $G=\left(f_{i}\right)_{\#}\left(G_{i}\right), i=1,2$. In particular, the only vectors with image in ${ }^{\mathrm{b}} T_{q} F=T_{q} G \oplus{ }^{\mathrm{b}} N_{q}(G ; F)$ must lie in the first two factors, namely $T_{p_{i}} G_{i} \oplus{ }^{\mathrm{b}} N_{p_{i}}\left(G_{i} ; F_{i}\right) \equiv{ }^{\mathrm{b}} T_{p_{i}} F_{i}$. Thus if

$$
{ }^{\mathrm{b}}\left(f_{1}\right)_{*}+{ }^{\mathrm{b}}\left(f_{2}\right)_{*}:{ }^{\mathrm{b}} T_{p_{1}} X_{1} \times{ }^{\mathrm{b}} T_{p_{2}} X_{2} \longrightarrow T_{q} Y
$$

is surjective, then

$$
{ }^{\mathrm{b}}\left(\left(f_{1}\right)_{\mid F_{1}}\right)_{*}+{ }^{\mathrm{b}}\left(\left(f_{2}\right)_{\mid F_{2}}\right)_{*}:{ }^{\mathrm{b}} T_{p_{1}} F_{1} \times{ }^{\mathrm{b}} T_{p_{2}} F_{2} \longrightarrow{ }^{\mathrm{b}} T_{q} F
$$

must be surjective. 
Proposition 11.4. If $f_{i}: X_{i} \longrightarrow Y, i=1,2$, are $b$-transversal maps, then the set-theoretic fiber product (11.1) is a union of interior binomial subvarieties

$$
D\left(F_{1}, F_{2}\right)=\operatorname{clos}\left(F_{1} \times_{Y} F_{2} \backslash \partial\left(F_{1} \times F_{2}\right)\right) \subset F_{1} \times F_{2}, \quad F_{i} \in \mathcal{M}\left(X_{i}\right) .
$$

Proof. Every point in $X_{1} \times_{Y} X_{2}$ lies in the interior of some $F_{1} \times F_{2}$ and hence in $D\left(F_{1}, F_{2}\right)$. It suffices to verify that the $D\left(F_{1}, F_{2}\right) \subset F_{1} \times F_{2}$ are either empty or interior binomial subvarieties. So we may restrict attention to the case $F_{i}=X_{i}$, and assume that $D\left(X_{1}, X_{2}\right)$ is nonempty (in particular, the $f_{i}$ are interior).

Suppose $X_{i} \ni p_{i} \longmapsto q \in Y$, and choose coordinates $\left(x^{\prime}, y^{\prime}\right)$ centered at $p_{1}$, $\left(x^{\prime \prime}, y^{\prime \prime}\right)$ centered at $p_{2}$, and $(\bar{x}, \bar{y})=\left(\bar{x}_{1}, \ldots, \bar{x}_{k}, \bar{y}_{k+1}, \ldots, \bar{y}_{n}\right)$ centered at $q$. The maps $f_{i}$ have the local form

$$
\begin{aligned}
& f_{1}:\left(x^{\prime}, y^{\prime}\right) \longmapsto\left(a_{1}\left(x^{\prime}, y^{\prime}\right)\left(x^{\prime}\right)^{\nu_{1}}, b_{1}\left(x^{\prime}, y^{\prime}\right)\right)=(\bar{x}, \bar{y}), \text { and } \\
& f_{2}:\left(x^{\prime \prime}, y^{\prime \prime}\right) \longmapsto\left(a_{2}\left(x^{\prime \prime}, y^{\prime \prime}\right)\left(x^{\prime \prime}\right)^{\nu_{2}}, b_{2}\left(x^{\prime \prime}, y^{\prime \prime}\right)\right)=(\bar{x}, \bar{y}) .
\end{aligned}
$$

Near $\left(p_{1}, p_{2}\right) \in X_{1} \times X_{2},(x, y)=\left(x^{\prime}, x^{\prime \prime}, y^{\prime}, y^{\prime \prime}\right)$ are local coordinates in terms of which

$$
D\left(X_{1}, X_{2}\right) \subset\left\{a_{1}\left(x^{\prime}, y^{\prime}\right)\left(x^{\prime}\right)^{\nu_{1}}=a_{2}\left(x^{\prime \prime}, y^{\prime \prime}\right)\left(x^{\prime \prime}\right)^{\nu_{2}}, b_{1}\left(x^{\prime}, y^{\prime}\right)=b_{2}\left(x^{\prime \prime}, y^{\prime \prime}\right)\right\},
$$

which can be written in the form

$$
D\left(X_{1}, X_{2}\right) \subset\left\{c_{i} x^{\gamma_{i}}=1 ; i=1, \ldots, n\right\}, \quad 0<c_{i} \in C^{\infty}\left(X_{1} \times X_{2}\right),
$$

where

$$
\left(c_{i}, \gamma_{i}\right)= \begin{cases}\left(\left(a_{1}\right)_{i} /\left(a_{2}\right)_{i},\left(\nu_{1} \oplus 0\right)_{i}-\left(0 \oplus \nu_{2}\right)_{i}\right), & i=1, \ldots, k \\ \left(\exp \left(\left(b_{1}\right)_{i}-\left(b_{2}\right)_{i}\right), 0\right), & i=k+1, \ldots, n .\end{cases}
$$

Thus it only remains to check the independence of the logarithmic differentials of the $c_{i} x^{\gamma_{i}}$. Consider the b-map $f_{1} \times f_{2}: X_{1} \times X_{2} \longrightarrow Y \times Y$. There is an exact sequence

$$
0 \rightarrow{ }^{\mathrm{b}} T_{q} Y \stackrel{\mathrm{b}^{\mathrm{b}}}{\rightarrow}{ }^{\mathrm{b}} T_{(q, q)}(Y \times Y) \rightarrow{ }^{\mathrm{b}} T_{q} Y \rightarrow 0
$$

where $\Delta: Y \longrightarrow Y \times Y$ is the diagonal inclusion and the subsequent map is the difference from ${ }^{\mathrm{b}} T_{(q, q)}(Y \times Y)={ }^{\mathrm{b}} T_{q} Y \times{ }^{\mathrm{b}} T_{q} Y$. The b-transversality condition means that ${ }^{\mathrm{b}}\left(f_{1} \times f_{2}\right)^{*}$ is injective as a map

$$
{ }^{\mathrm{b}}\left(f_{1} \times f_{2}\right)^{*}:{ }^{\mathrm{b}} T_{q}^{*} Y \longrightarrow{ }^{\mathrm{b}} T_{\left(p_{1}, p_{2}\right)}^{*}\left(X_{1} \times X_{2}\right) .
$$

This can be identified with the map

$$
{ }^{\mathrm{b}} f_{1}^{*}-{ }^{\mathrm{b}} f_{2}^{*}:{ }^{\mathrm{b}} T_{q}^{*} Y \longrightarrow{ }^{\mathrm{b}} T_{\left(p_{1}, p_{2}\right)}^{*}\left(X_{1} \times X_{2}\right),
$$

which is similarly injective. Taking the coordinate basis $\left\{d \bar{x}_{i} / \bar{x}_{i}, d \bar{y}_{j}\right\}$ for ${ }^{\mathrm{b}} T_{q}^{*} Y$, we obtain that

$$
\left\{\left({ }^{\mathrm{b}} f_{1}^{*}-{ }^{\mathrm{b}} f_{2}^{*}\right) d \bar{x}_{i} / \bar{x}_{i},\left({ }^{\mathrm{b}} f_{1}^{*}-{ }^{\mathrm{b}} f_{2}^{*}\right) d \bar{y}_{j}\right\}
$$

is independent. Observe however that

$$
\begin{aligned}
\left({ }^{\mathrm{b}} f_{1}^{*}-{ }^{\mathrm{b}} f_{2}^{*}\right) d \bar{x}_{i} / \bar{x}_{i} & =d \log \left(\left(a_{1} x^{\prime \nu_{1}}\right)_{i}\right)-d \log \left(\left(a_{2} x^{\prime \prime \nu_{2}}\right)_{i}\right) \\
& =d \log \left(c_{i} x^{\gamma_{i}}\right), \quad i=1, \ldots, k,
\end{aligned}
$$


and

$$
\begin{aligned}
\left({ }^{\mathrm{b}} f_{1}^{*}-{ }^{\mathrm{b}} f_{2}^{*}\right) d \bar{y}_{i} & =d\left(b_{1}\right)_{i}-d\left(b_{2}\right)_{i} \\
& =d \log \left(\exp \left(\left(b_{1}\right)_{i}-\left(b_{2}\right)_{i}\right)\right) \\
& =d \log \left(c_{i} x^{\gamma_{i}}\right), \quad i=k+1, \ldots, n .
\end{aligned}
$$

We conclude that $D\left(X_{1}, X_{2}\right)$ has a covering by local binomial structures and is therefore an interior binomial subvariety.

While $X_{1} \times_{Y} X_{2}$ may therefore be a complicated and quite singular space, any smooth maps factoring through it must actually factor through one of the subvarieties $D\left(F_{1}, F_{2}\right)$.

Proposition 11.5. If $g_{i}: Z \longrightarrow X_{i}$ are b-maps from a connected smooth manifold such that $f_{1} \circ g_{1}=f_{2} \circ g_{2}$, then the maps $g_{i}: Z \longrightarrow X_{i}$ factor through a canonical b-map $h: Z \longrightarrow D\left(F_{1}, F_{2}\right)$ for some $D\left(F_{1}, F_{2}\right)$ :

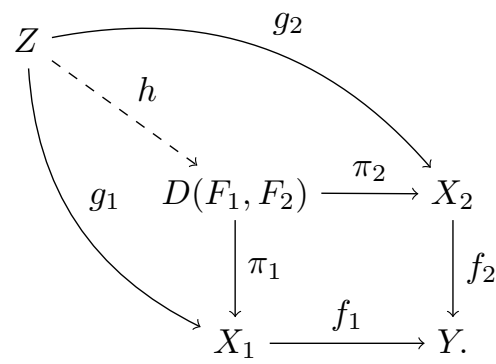

While the map $h$ exists and may be chosen canonically, it is not necessarily unique, since there may be several pairs $\left(F_{1}, F_{2}\right)$ such that $Z$ factors through $D\left(F_{1}, F_{2}\right)$.

Proof. Let $F_{1}, F_{2}$ be the minimal (largest codimension) faces such that $g_{i}: Z \longrightarrow F_{i}$ are interior b-maps. Then $g_{1} \times g_{2}: Z \longrightarrow F_{1} \times F_{2} \subset X_{1} \times X_{2}$ is an interior b-map, and as such

$$
g_{1} \times g_{2}: Z \backslash \partial Z \longrightarrow F_{1} \times F_{2} \backslash \partial\left(F_{1} \times F_{2}\right) .
$$

On the other hand, as a map of sets, $g_{1} \times g_{2}$ factors through (the set-theoretic) $F_{1} \times_{Y} F_{2}$ by the assumption that $f_{1} \circ g_{1}=f_{2} \circ g_{2}$. Finally, it follows by continuity and taking the closure of the intersection with the interior of $F_{1} \times F_{2}$ that

$$
h=g_{1} \times g_{2}: Z \longrightarrow D\left(F_{1}, F_{2}\right) \subset X_{1} \times X_{2},
$$

which is by definition a b-map into an interior binomial subvariety.

Note that the monoidal complex $\mathcal{P}_{D\left(F_{1}, F_{2}\right)}$ consists of monoids of the form $\sigma_{G_{1}} \times_{\sigma_{G}} \sigma_{G_{2}}$, where $\sigma_{G_{i}} \in \mathcal{P}_{F_{i}}$, and $G=\left(f_{1}\right)_{\#}\left(G_{1}\right) \cap\left(f_{2}\right)_{\#}\left(G_{2}\right)$. Indeed, if $F$ is a boundary face of $D\left(F_{1}, F_{2}\right)$, given by a component of $D\left(F_{1}, F_{2}\right) \cap G_{1} \times G_{2}$ for $G_{1} \times G_{2} \in \mathcal{M}\left(X_{1} \times X_{2}\right)$, then

$$
\sigma_{F}=\sigma_{G_{1} \times G_{2}} \cap{ }^{\mathrm{b}} N F=\sigma_{G_{1}} \times_{\sigma_{G}} \sigma_{G_{2}} .
$$

It is generally not true that $\mathcal{P}_{D\left(F_{1}, F_{2}\right)}$ is equal to $\mathcal{P}_{F_{1}} \times_{\mathcal{P}_{Y}} \mathcal{P}_{F_{2}}$ as might at first be expected, since $D\left(F_{1}, F_{2}\right) \cap G_{1} \times G_{2}$ may be empty or may have multiple components, while $\sigma_{G_{1}} \times_{\sigma_{G}} \sigma_{G_{2}}$ is non-trivial and appears exactly once in $\mathcal{P}_{F_{1}} \times_{\mathcal{P}_{Y}} \mathcal{P}_{F_{2}}$. 
We next present two results guaranteeing the existence of smooth fiber products in certain circumstances, under the assumption that the $\mathcal{P}_{D\left(F_{1}, F_{2}\right)}$ are smooth. Recall that a b-map $f: X \longrightarrow Y$ is said to be b-normal if for all $F \in \mathcal{M}(X)$, the b-differential

$$
{ }^{\mathrm{b}} f_{*}:{ }^{\mathrm{b}} N F \longrightarrow{ }^{\mathrm{b}} N f_{\#}(F)
$$

is surjective. In particular the codimension of $f_{\#}(F)$ can be at most that of $F$.

We also note that the set $\mathcal{B}=\left\{D\left(F_{1}, F_{2}\right) \neq \emptyset\right\}$ of non-empty varieties inherits an order from the one on $\mathcal{M}\left(X_{1}\right) \times \mathcal{M}\left(X_{2}\right)$, where $D\left(F_{1}, F_{2}\right) \leq D\left(G_{1}, G_{2}\right)$ if $F_{i} \leq G_{i}$ for $i=1,2$. In particular if $D\left(X_{1}, X_{2}\right) \neq \emptyset$, then it is a maximal element of $\mathcal{B}$.

Theorem 11.6 (Existence of smooth fiber products). Let $f_{i}: X_{i} \longrightarrow Y, i=1,2$, be b-transversal, and suppose that $\sigma_{F_{1}} \times_{\sigma_{G}} \sigma_{F_{2}}$ is smooth for all $F_{i} \in \mathcal{M}\left(X_{i}\right)$ where $G=\left(f_{1}\right)_{\#}\left(F_{1}\right) \cap\left(f_{2}\right)_{\#}\left(F_{2}\right) \in \mathcal{M}(Y)$.

(a) If the $f_{i}$ are interior b-maps, then $\left[D\left(X_{1}, X_{2}\right) ; \mathcal{P}_{D\left(X_{1}, X_{2}\right)}\right]$ is a smooth fiber product in the category of manifolds with corners and interior b-maps.

(b) If $\mathcal{B}=\left\{D\left(F_{1}, F_{2}\right) \neq \emptyset\right\}$ has a maximal element $D\left(G_{1}, G_{2}\right)$ (in particular if $D\left(X_{1}, X_{2}\right)$ is nonempty $)$, and if the $f_{i}$ are b-normal, then $\left[D\left(G_{1}, G_{2}\right) ; \mathcal{P}_{D\left(G_{1}, G_{2}\right)}\right]$ is a smooth fiber product in the category of manifolds with corners and general b-maps.

As noted in the previous section, even when the smooth fiber product exists, it may not be smoothly embedded in $X_{1} \times X_{2}$. The assumption that $\mathcal{B}$ have a maximal element is important; without this condition it is easy to construct counterexamples where the $f_{i}: X_{i} \longrightarrow Y$ are b-transversal embeddings whose fiber product (which is just the intersection in this case) is a union of proper boundary faces.

Proof. The monoidal condition ensures that each of the complexes $\mathcal{P}_{D\left(F_{1}, F_{2}\right)}$ is smooth. For (国), interior b-maps must factor uniquely through $D\left(X_{1}, X_{2}\right)$, and hence by Theorem 10.5 through the universal resolution $\left[D\left(X_{1}, X_{2}\right), \mathcal{P}_{D\left(X_{1}, X_{2}\right)}\right]$. If $D\left(X_{1}, X_{2}\right)$ is empty, then there are no non-empty manifolds $Z$ mapping through the $X_{i}$ to $Y$.

For (b) we show, using b-normality, that each non-empty binomial variety $D\left(F_{1}, F_{2}\right)$ is contained in the maximal one; it then follows that any $Z \longrightarrow X_{i}$ must factor uniquely through the smooth manifold $\left[D\left(G_{1}, G_{2}\right) ; \mathcal{P}_{D\left(G_{1}, G_{2}\right)}\right]$ by Theorem 10.5. It is sufficient to show that the interior $\left(F_{1} \times_{Y} F_{2}\right) \backslash \partial\left(F_{1} \times F_{2}\right)$ is contained in $D\left(G_{1}, G_{2}\right)$ since the latter is closed, and also to assume that the $f_{i}$ are interior b-maps, replacing the $X_{i}$ by $G_{i}$ and $Y$ by $\left(f_{1}\right)_{\#}\left(G_{1}\right)=\left(f_{2}\right)_{\#}\left(G_{2}\right)$ if necessary.

Thus suppose $\left(p_{1}, p_{2}\right) \in\left(F_{1} \times_{Y} F_{2}\right) \backslash \partial\left(F_{1} \times F_{2}\right)$, so in particular $p_{i} \in \stackrel{\circ}{F}_{i}$ for $i=1,2$, and that $D\left(X_{1}, X_{2}\right)$ is non-empty, hence maximal. The b-normality condition ensures that there exist local boundary defining functions $\left(x_{1}^{\prime}, \ldots, x_{k_{1}}^{\prime}\right)$ and $\left(x_{1}^{\prime \prime}, \ldots, x_{k_{2}}^{\prime \prime}\right)$ for $F_{1}$ and $F_{2}$, respectively, near $p_{1}$ and $p_{2}$ so that, if $\left(\bar{x}_{1}, \ldots, \bar{x}_{k}\right)$ are boundary defining functions for $F=\left(f_{1}\right)_{\#}\left(F_{1}\right)=\left(f_{2}\right)_{\#}\left(F_{2}\right)$ near $q=f_{1}\left(p_{1}\right)=$ $f_{2}\left(p_{2}\right)$, then

$$
f_{1}^{*}(\bar{x})=x^{\prime \nu_{1}}, \quad f_{2}^{*}(\bar{x})=x^{\prime \prime \nu_{2}} .
$$

Indeed, as in the proof of Lemma 8.2 we may take any set $x^{\prime}=\left(x_{1}^{\prime}, \ldots, x_{k_{1}}^{\prime}\right)$ of boundary defining functions for $F_{1}$, for which $f_{1}^{*}(\bar{x})=a_{1} x^{\prime \nu_{1}}$ initially, with $0<a_{1} \in\left(C^{\infty}\left(X_{1}\right)\right)^{k}$. Then by surjectivity of ${ }^{\mathrm{b}}\left(f_{1}\right)_{*}$ as represented by the matrix $\nu_{1}^{\mathrm{T}}$, we may replace these by $b_{1}^{-1} x^{\prime}$ where $0<b_{1} \in\left(C^{\infty}(Y)\right)^{k_{1}}$ satisfies $a_{1}=b_{1}^{\nu_{1}}$. 
A similar procedure gives the coordinates $x^{\prime \prime}$ near $p_{2}$. It follows that, locally, the defining equations for the fiber product have the form

$$
\begin{aligned}
x^{\prime\left(\nu_{1}\right)_{i}} & =x^{\prime \prime\left(\nu_{2}\right)_{i}}, & & i=1, \ldots, k, \\
f_{j}\left(x^{\prime}, x^{\prime \prime}, y^{\prime}, y^{\prime \prime}\right) & =0, & j & =k+1, \ldots, n,
\end{aligned}
$$

which are satisfied at $\left(p_{1}, p_{2}\right)=(0,0,0,0)$. By Proposition 11.3, the differentials $d_{y^{\prime}, y^{\prime \prime}} f_{j}\left(0,0, y^{\prime}, y^{\prime \prime}\right), j=k+1, \ldots, n$, are independent, and therefore they remain so for small $\left(x^{\prime}, x^{\prime \prime}\right)$. Moreover, the b-normality condition ensures that the equations $x^{\prime\left(\nu_{1}\right)_{i}}=x^{\prime \prime\left(\nu_{2}\right)_{i}}, i=1, \ldots, k$, are completely decoupled. Indeed, for each hypersurface $H_{i}^{\prime} \in \mathcal{M}\left(X_{1}\right)$, given locally by $x_{i}^{\prime}=0$, the image $\left(f_{1}\right)_{\#}\left(H_{i}^{\prime}\right) \in \mathcal{M}(Y)$ must be a hypersurface by b-normality and therefore $x_{i}^{\prime}$ can appear in at most one of the equations, and similarly for $x_{i}^{\prime \prime}$. It follows from the implicit function theorem that there are nearby solutions to (11.3) in any neighborhood of $(0,0,0,0)$ with all the $x^{\prime}$ and $x^{\prime \prime}$ positive, and therefore $\left(p_{1}, p_{2}\right)$ lies in the closure of $\left(X_{1} \times_{Y} X_{2}\right) \backslash \partial\left(X_{1} \times X_{2}\right)$, which is $D\left(X_{1}, X_{2}\right)$.

In general, even if the $\mathcal{P}_{D\left(F_{1}, F_{2}\right)}$ are smooth, $X_{1} \times_{Y} X_{2} \subset X_{1} \times X_{2}$ is a nonsmooth variety which is in some sense 'reducible', being the union of the smooth varieties $D\left(F_{1}, F_{2}\right)$. This may be resolved by replacing $X_{1} \times_{Y} X_{2}$ by the disjoint union of the $D\left(F_{1}, F_{2}\right)$, with the cost of losing one of the universal properties of the fiber product.

We define a weak fiber product of maps $f_{i}: X_{i} \longrightarrow Y$ to be a space $D$ with maps $\pi_{i}: D \longrightarrow X_{i}$ such that if $g_{i}: Z \longrightarrow X_{i}$ are maps from an additional space satisfying $f_{1} \circ g_{1}=f_{2} \circ g_{2}$, then there exists a canonical (but not necessarily unique) map $g: Z \longrightarrow D$ with $g_{i}=\pi_{i} \circ g$. A weak fiber product is not necessarily unique up to isomorphism.

Under b-transversality alone, the space

$$
D=\bigsqcup_{F_{i} \in \mathcal{M}\left(X_{i}\right), i=1,2} D\left(F_{1}, F_{2}\right)
$$

serves as a fiber product in the category of interior binomial subvarieties with its attendant resolution theory, which we summarize below.

Theorem 11.7. Let $f_{i}: X_{i} \longrightarrow Y, i=1,2$, be b-transverse maps and define $D$ as above.

(a) For every smooth refinement $\mathcal{R}_{D} \longrightarrow \mathcal{P}_{D}$ there is a smooth manifold with corners $\left[D ; \mathcal{R}_{D}\right]$, with maps $h_{i}:\left[D ; \mathcal{R}_{D}\right] \longrightarrow X_{i}$ forming a commutative square with $f_{i}: X_{i} \longrightarrow Y$ :

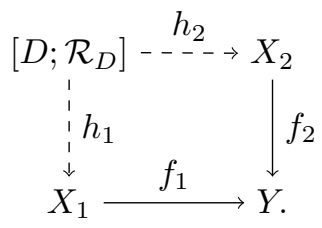

(b) Any two such resolutions $\left[D ; \mathcal{R}_{i}\right], i=1,2$, have a mutual smooth resolution, which is to say a third manifold $\left[D ; \mathcal{R}_{0}\right]$ with maps $\left[D ; \mathcal{R}_{0}\right] \longrightarrow\left[D ; \mathcal{R}_{i}\right], i=1,2$, 
forming commutative diagrams with the maps $\left[D ; \mathcal{R}_{i}\right] \longrightarrow X_{j}$ :

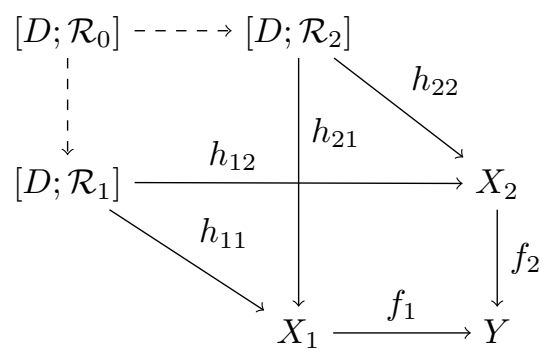

(c) If $Z$ is a manifold with maps $g_{i}: Z \longrightarrow X_{i}$ such that $f_{1} \circ g_{1}=f_{2} \circ g_{2}$, and if the morphism $\mathcal{P}_{Z} \longrightarrow \mathcal{P}_{D}$ factors through $\mathcal{R}_{D} \longrightarrow \mathcal{P}_{D}$, then there is a canonical map $g: Z \longrightarrow\left[D ; \mathcal{R}_{D}\right]$ such that $h_{i} \circ g=g_{i}$ :

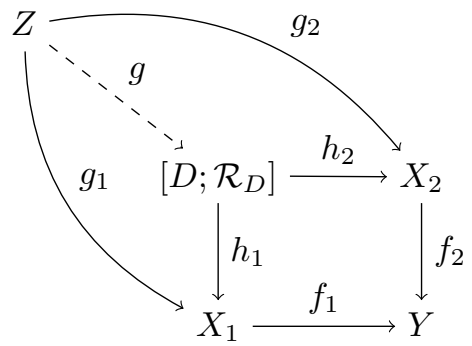

(d) Given a manifold $Z$ with maps $g_{i}: Z \longrightarrow X_{i}$ such that $f_{1} \circ g_{1}=f_{2} \circ g_{2}$, for any resolution $\left[D ; \mathcal{R}_{D}\right] \longrightarrow D$ there exists a generalized blow-up $\beta:[Z ; \mathcal{R}] \longrightarrow Z$ and a canonical map $g:[Z ; \mathcal{R}] \longrightarrow\left[D ; \mathcal{R}_{D}\right]$ such that $h_{i} \circ g=g_{i} \circ \beta$ :

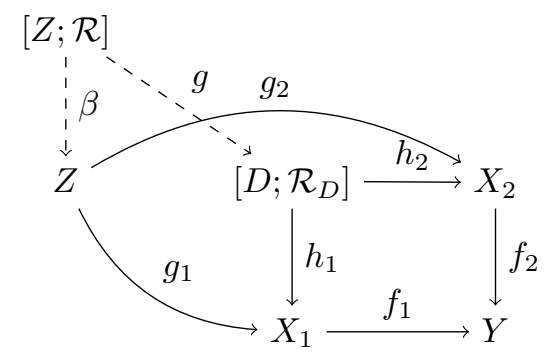

For a manifold $Z$ with maps $g_{i}: Z \longrightarrow X_{i}$, it is not generally true that there exists a resolution $\left[D ; \mathcal{R}_{D}\right] \longrightarrow D$ through which $Z$ factors, without blowing up the domain. Also note that if the $f_{i}$ are b-normal and $D\left(X_{1}, X_{2}\right) \neq \emptyset$, then 'canonical' can be replaced by 'unique' in (Ic) and (d), since as in the proof of Theorem 11.6, $D \equiv D\left(X_{1}, X_{2}\right)$ in this case.

Proof. The smooth manifold $\left[D ; \mathcal{R}_{D}\right]$ is well defined by the results in Section 10 , giving (国). (b) follows from the existence of mutual refinements, letting $\mathcal{R}_{0}$ be a smooth refinement of $\mathcal{R}_{1} \times_{\mathcal{P}_{D}} \mathcal{R}_{2}$. (IC) follows from Proposition 11.5 and Theorem 6.3. and (d) follows from Theorem 6.5, letting $\mathcal{R}$ be a smooth refinement of $\mathcal{P}_{Z} \times \mathcal{P}_{D}$ $\mathcal{R}_{D}$. 

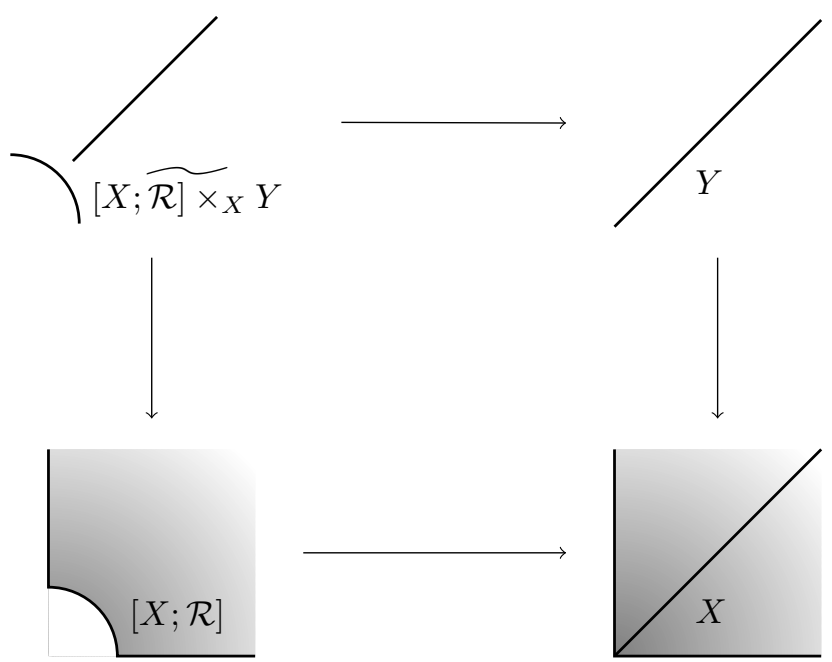

FiguRE 5. The fiber product of a generalized blow-down and the inclusion of a manifold as an interior binomial subvariety.

In particular, if $\mathcal{P}_{D}$ is already smooth, we may take $\mathcal{R}_{D}=\mathcal{P}_{D}$ in (ㄷ) and obtain:

Corollary 11.8. If $f_{i}: X_{i} \longrightarrow Y$ are $b$-transverse maps and if in addition $\sigma_{F_{1}} \times_{\sigma_{G}}$ $\sigma_{F_{2}}$ is a smooth monoid whenever $G=\left(f_{1}\right)_{\#}\left(F_{1}\right) \cap\left(f_{2}\right)_{\#}\left(F_{2}\right) \in \mathcal{M}(Y)$, then as a union of smooth manifolds,

$$
\widetilde{X_{1} X_{Y}} X_{2}=\bigsqcup_{F_{1}, F_{2}}\left[D\left(F_{1}, F_{2}\right) ; \mathcal{P}_{D\left(F_{1}, F_{2}\right)}\right]
$$

is a weak fiber product in the category of manifolds with corners.

Some of the situations we have already considered are interesting examples of weak fiber products.

Example 11.9 (A blow-up and a binomial subvariety). Let $Y$ be a smooth manifold which is included in $X$ as an interior binomial variety, and let $X_{1}=[X ; \mathcal{R}] \longrightarrow X$ be a generalized blow-down. Since $i: Y \longleftrightarrow X$ is injective, the fiber product $X_{1} \times_{X} Y$ can be identified with $\beta^{-1}(Y) \subset X_{1}$. The interior subvariety $D\left(Y, X_{1}\right)$ is just the lift/proper transform $\beta^{\#}(Y)$, which we showed to be an interior binomial subvariety in Section 10. Observe, however, that $\beta^{-1}(Y)$ generally contains other subvarieties as well, namely $F \cap \beta^{-1}(G)$, where $F \in \mathcal{M}\left(X_{1}\right)$ and $G \in \mathcal{M}(D)$. See Figure 5 .

Example 11.10 (Two blow-ups). We leave it as an exercise for the reader to show that the fiber product of two blow-down maps $\beta_{i}:\left[X ; \mathcal{R}_{i}\right] \longrightarrow X, i=1,2$, is a subvariety with the monoidal complex $\mathcal{R}_{1} \times_{\mathcal{P}_{X}} \mathcal{R}_{2}$. By Proposition 5.2 this is a (not necessarily smooth) refinement of $\mathcal{P}_{X}$, which can be identified with the intersection complex $\mathcal{R}_{1} \cap \mathcal{R}_{2}=\left\{\sigma_{F_{1}} \cap \sigma_{F_{2}} \subset \sigma_{F} ; \sigma_{F_{i}} \in \mathcal{R}_{i}(F)\right\}$. It is not smooth in general.

Example 11.11 (Joyce's fiber products). In [Joy09, Joyce proposes a category of manifolds with corners in which the morphisms are what might be called 'simple 
b-maps' $f: X \longrightarrow Y$ which have the property that whenever

$$
f^{*} \mathcal{I}_{H}=\prod_{G \in \mathcal{M}_{1}(X)} \mathcal{I}_{G}^{\alpha(H, G)}, \quad H \in \mathcal{M}_{1}(Y)
$$

the $\alpha(\cdot, \cdot)$ are either zero or one, and furthermore for every $H$ there is at most one $G$ such that $\alpha(H, G) \neq 0$ (which is equivalent to b-normality). As a consequence the morphisms

$$
f_{\natural}: \sigma_{F} \longrightarrow \sigma_{f_{\#}(F)}
$$

are always injective, with images which have orthogonal generators $\left\{v_{i}\right\}$ all of whose components are zero or one.

Joyce defines a transversality condition in terms of the ordinary differential. Though he does not use the b-differential, his is an iterated transversality condition on boundary faces analogous to Proposition 11.3. Joyce shows that for two transversal maps in his sense, the set-theoretic fiber product is a manifold with corners.

This also follows from Theorem [11.6. Indeed, for b-transversal simple b-maps, the monoids $\sigma_{F_{1}} \times_{\sigma_{G}} \sigma_{F_{2}}$ can be identified with the intersections $\sigma_{F_{1}} \cap \sigma_{F_{2}} \subset \sigma_{G}$ by injectivity, and with the properties above, these intersections are automatically smooth.

\section{ACKNOWLEDGEMENTS}

The authors would like to thank Dan Abramovich, Pierre Albin, William Gillam, Daniel Grieser, Samouil Molcho and Michael Singer for helpful discussions during the preparation of this manuscript, the referee for many insightful comments, and Dominic Joyce for pointing out a mistake in an earlier version of the theorem on fiber products. The authors should also like to point out the important role in the origins of this work played by Umut Varolgunes and Jonathan Zhu who investigated the resolution of sums of positive monomials in the model, Euclidean, case in an 'Undergraduate Research Opportunity' at MIT.

\section{REFERENCES}

[DCP83] C. De Concini and C. Procesi, Complete symmetric varieties, II; Intersection theory, Algebraic groups and related topics, Adv. Stud. Pure Math, vol. 6, Kyoto/Nagoya, 1983, pp. 481-513.

[Ful93] William Fulton, Introduction to toric varieties, Annals of Mathematics Studies, vol. 131, Princeton University Press, Princeton, NJ, 1993. The William H. Roever Lectures in Geometry. MR.1234037 (94g:14028)

[Joy09] D. Joyce, On manifolds with corners, Adv. Lect. Math. (ALM), 21, Int. Press, Somerville, MA, 2012. MR.3077259

[Kat94] Kazuya Kato, Toric singularities, Amer. J. Math. 116 (1994), no. 5, 1073-1099, DOI 10.2307/2374941. MR1296725 (95g:14056)

[KKMSD73] G. Kempf, Finn Faye Knudsen, D. Mumford, and B. Saint-Donat, Toroidal embeddings. I, Lecture Notes in Mathematics, Vol. 339, Springer-Verlag, Berlin, 1973. MR0335518 (49 \#299)

[Mel] R. B. Melrose, Differential analysis on manifolds with corners, In preparation, partially available at http://math.mit.edu/ $\mathrm{rbm} /$ book.html.

[Mel92] Richard B. Melrose, Calculus of conormal distributions on manifolds with corners, Internat. Math. Res. Notices 3 (1992), 51-61, DOI 10.1155/S1073792892000060. MR.1154213 (93i:58148)

[Ogu06] A. Ogus, Lectures on logarithmic algebraic geometry, Notes available at http://math.berkeley.edu/〜ogus/preprints/log_book/logbook.pdf (2006). 
[Tei] Bernard Teissier, Monomial ideals, binomial ideals, polynomial ideals, Trends in commutative algebra, Math. Sci. Res. Inst. Publ., vol. 51, Cambridge Univ. Press, Cambridge, 2004, pp. 211-246, DOI 10.1017/CBO9780511756382.008. MR 2132653 (2006c:13032)

Department of Mathematics, Brown University, Providence, Rhode Island 02912

E-mail address: ckottke@math.brown.edu

Current address: Department of Mathematics, Northeastern University, Boston, Massachusetts 02115

E-mail address: c.kottke@neu.edu

Department of Mathematics, Massachusetts Institute of Technology, Cambridge, MassachusetTs 02139

E-mail address: rbm@math.mit.edu 